\title{
Thrombinoscopy Revisited
}

Citation for published version (APA):

Moorlag, M. (2019). Thrombinoscopy Revisited. [Doctoral Thesis, Maastricht University]. Maastricht University. https://doi.org/10.26481/dis.20190517mm

Document status and date:

Published: 01/01/2019

DOI:

10.26481/dis.20190517mm

Document Version:

Publisher's PDF, also known as Version of record

\section{Please check the document version of this publication:}

- A submitted manuscript is the version of the article upon submission and before peer-review. There can be important differences between the submitted version and the official published version of record.

People interested in the research are advised to contact the author for the final version of the publication, or visit the DOI to the publisher's website.

- The final author version and the galley proof are versions of the publication after peer review.

- The final published version features the final layout of the paper including the volume, issue and page numbers.

Link to publication

\footnotetext{
General rights rights.

- You may freely distribute the URL identifying the publication in the public portal. please follow below link for the End User Agreement:

www.umlib.nl/taverne-license

Take down policy

If you believe that this document breaches copyright please contact us at:

repository@maastrichtuniversity.nl

providing details and we will investigate your claim.
}

Copyright and moral rights for the publications made accessible in the public portal are retained by the authors and/or other copyright owners and it is a condition of accessing publications that users recognise and abide by the legal requirements associated with these

- Users may download and print one copy of any publication from the public portal for the purpose of private study or research.

- You may not further distribute the material or use it for any profit-making activity or commercial gain

If the publication is distributed under the terms of Article $25 \mathrm{fa}$ of the Dutch Copyright Act, indicated by the "Taverne" license above, 


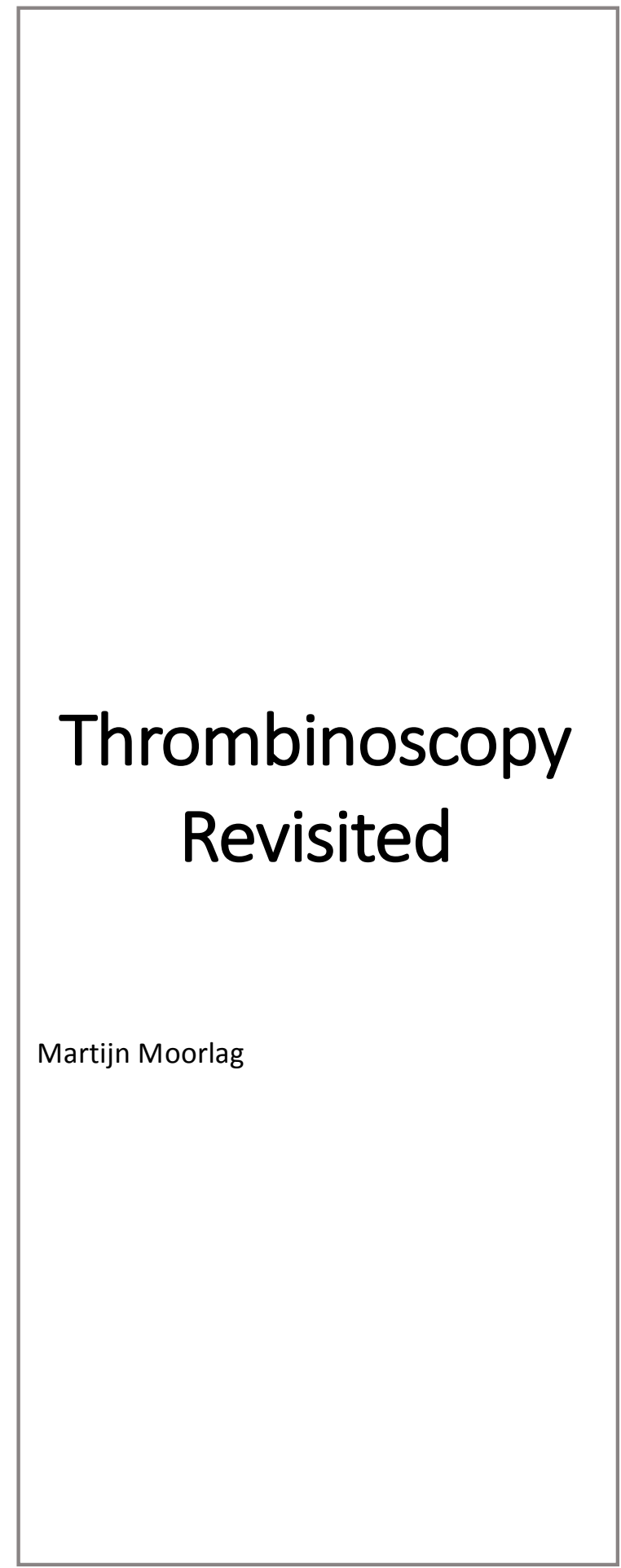


(C) Martijn Moorlag 2019

No part of this book may be reproduced or transmitted in any form or by any means without prior permission in writing by the author, or when appropriate, by the publishers of the publication.

Cover: P\&P-design adapted from the PhD-thesis of Suzette Béguin.

Printed by: Ipskamp printing

ISBN: 978-94-028-1533-7 


\title{
Thrombinoscopy Revisited
}

\author{
PROEFSCHRIFT \\ ter verkrijging van de graad van doctor aan de Universiteit Maastricht, \\ op gezag van de Rector Magnificus, prof. dr. Rianne M. Letschert \\ volgens het besluit van het College van Decanen, \\ In het openbaar te verdedigen op vrijdag 17 mei 2019 om 13:45 uur
}

door

Martijn Moorlag

Geboren op 18 juni 1986 te Groningen 


\section{Promotores:}

Prof. dr. T.M. Hackeng

(de jure)

Prof. dr. H.C. Hemker

(de facto)

\section{Beoordelingscommissie:}

Prof. dr. C.P.M. Reutelingsperger

(voorzitter)

Prof. dr. M.P. van Dieijen-Visser

Prof. dr. R.A.S. Ariens

Prof. dr. J. Pieters

(University of Basel)

Dr. ir. D.T.S. Rijkers

(Universiteit Utrecht) 


\section{Contents}

$\begin{array}{lll}\text { Chapter } 1 & \text { General introduction } & 7\end{array}$

Chapter 2 Near patient thrombin generation in 27 patients undergoing elective cardiac surgery

Chapter 3 Thrombin generation in zebrafish blood

Chapter 4 On the causes of inter-laboratory variability in calibrated thrombin generation measurement

Chapter 5 The effects of temperature on the 75 thrombin generation experiment

Chapter 6 The relation between thrombin concentration and reaction velocity as measured from the conversion of fluorogenic substrate.

Chapter 7 Indirect measurement of initial reaction rates from progress curves, its application to investigate the influence of albumin on the interaction of thrombin and the $\alpha 2$ macroglobulin-thrombin complex with the fluorogenic substrate ZGGR-AMC.

Chapter 8 Correction of the effect of the fluorogenic thrombin substrate used in calibrated automated thrombography on physiological thrombin generation

Chapter 9 General discussion and conclusions

Chapter 10 Nederlandse samenvatting

Appendix I A blueprint for the advanced thermoregulation of the Ascent reader.

Appendix II Valorisation 
Appendix Acknowledgements

183

III

Appendix Scientific output And Curriculum Vitae

189

IV 

Chapter 1

General introduction 


\section{Preface}

In the assessment of the blood coagulation system, researchers have an everexpanding toolkit at their disposal. They (re-)design assays to investigate coagulation e.g. under the influence of blood flow ${ }^{1,2}$, with and without blood platelets ${ }^{3,4}$ in whole blood and/or in the presence of endothelial cells ${ }^{5}$. On the contrary, clinicians are not exactly spoiled for choice, since most of their assays being based on the same clotting time measurements that date back to the middle of the previous century. In other words, there is a need for the clinical implementation of more insightful blood coagulation assays and judging from the number of assays used in research settings there should be sufficient choice.

This thesis focusses on one test in particular, the thrombin generation test, which was first presented by Hemker ${ }^{3}$. The test has been around for over 15 years now and despite its potential as a clinically relevant measurement, still has not been implemented in the clinic. One of the main reasons that halted further clinical development was the high inter-laboratory variability that has been reported over the years ${ }^{6}$.

The initial goal of this work was to create a possibility for the clinical implementation of the thrombin generation assay. The experience gained from this work led us to take a step back and identify the sources of variability in the thrombin generation assay, to use this knowledge for the proposition of solutions and to integrate this in the design of a method which is robust enough for clinical implementation. 


\section{The coagulation system}

This text is aimed at giving an overview of the coagulation system within which new insights can be incorporated. The description leaves room for the ambiguities of the coagulation system where many of its components have multiple, and sometimes contradictory functions in maintaining the dynamic balance of haemostasis.

Ever since the first description of pathological haemostasis by Huang $\mathrm{Ti}$ in approximately 2650 B. $C^{7}$, the coagulation system and many of its intricacies and interactions have been the topic of (scientific) research. Despite this early observation, it was not until a couple of thousand years later that a more deliberate investigation led to further insight in the formation of venous thrombi. One of the earliest aetiologies stems from Wiseman (1686) with his description of venous occlusion by blood clots through both stasis and hypercoagulability.

Throughout the last decades our understanding of the system and the interactions of its constituents has become broader and broader, thanks to advances in technology facilitating more sophisticated approaches.

It is now known that the blood coagulation system consists of a series of zymogens and their pro/cofactors, most of which circulate in an inactive form. Thrombin (Flla) is the central enzyme within this system ${ }^{9}$. It is, amongst other things, responsible for the cleavage of fibrinogen to fibrin, the main constituent of a blood clot. Thrombin is formed after the cleavage of its zymogen prothrombin (FII). The level of conservation of thrombin throughout evolution highlights the importance of prothrombin in coagulation and ultimately in survival. The sequence identity between human and hagfish (one of the oldest ancestors still in existence, dating back some 300 million years) is $53 \%{ }^{10}$.

Prothrombin is converted into thrombin after it has been cleaved at two different sites $^{11}$. At the onset of coagulation only a small amount of thrombin is needed to form a blood clot. These trace amounts of thrombin assist in the formation of larger amounts of thrombin via several feedback mechanisms by which it enhances its own propagation. Besides stimulating its own formation, thrombin also activates its own inhibitors. These inhibitors put a halt to the formation of thrombin, ensuring containment of the process of coagulation.

There are several ways in which coagulation can be initiated e.g. by a rupture of an atherosclerotic plaque or by a wound that ruptures a vesse $\left.\right|^{12}$. When the vessel wall is damaged, it exposes the blood to TF, a protein bound to the membrane of perivascular cells. This prompts circulating factor VII (FVII) to bind the membrane 
adjacent the now exposed TF. In circulation FVII already has some activity, but when it interacts with TF this activity is greatly intensified ${ }^{13}$. Through this interaction FVII is now able to activate the proenzyme factor X (FX). Activated FX (FXa) in turn, is able to fully activate FVII into FVIla hereby speeding up its own formation even more. These feedback reactions lead to an explosion of active proteins.

FXa is able to convert prothrombin into meizothrombin, which is an active form of thrombin that remains membrane bound ${ }^{14,15}$. Being membrane bound, meizothrombin is in a perfect position to activate co-factor $V(F V)$ that binds the membrane as well. Activated FV (FVa) forms a complex with FXa and calcium on a phospholipid surface ${ }^{16}$ The formation of this so called prothrombinase complex increases the activation of prothrombin over a 1000 times compared to FXa alone ${ }^{17,18}$. The accelerated cleavage by the prothrombinase complex leads to a burst of thrombin.

With such a prolific burst of procoagulant activity, it is easily understood that inactivation of the coagulation system is just as crucial in maintaining a haemostatic balance as activation is. The coagulation system is best viewed as a dynamic equilibrium that is allowed to fall to one side in case of e.g. injury, but which is rapidly restored when coagulation is no longer necessary. When FXa circulates freely, it quickly interacts with a protein called tissue factor pathway inhibitor (TFPI). This FXa - TFPI complex is an excellent inhibitor of the TF-VIla complex ${ }^{19,20}$. Moreover, $\mathrm{FVa}$ (when it is not bound to a membrane) is also a potent inhibitor of the TF-VIla complex ${ }^{21}$. Therefore, FXa and FVa propagate their own generation when bound to a membrane and they become inhibitors of coagulation when in solution.

This very efficient inhibitory mechanism only allows FXa to be active for a brief period of time. Consequently, coagulation would not be able to reach the explosive state under low TF conditions, if it were not for an additional pathway. This pathway named the Josso loop ${ }^{22}$ after its discoverer François Josso (1927-1981), is triggered by the FVII-TF complex through the activation of FIX. Activated FIX (FIXa) is able to activate FX when it is bound to a phospholipid membrane and when it is in complex with activated factor VIII (FVIIIa), this FIXa-VIIIa complex is also known as the "tenase complex" 2324.

Factor VIII (FVIII) is present in plasma bound to von Willebrand factor (VWF). As long as FVIII is bound to VWF it is unable to bind to a phospholipid membrane and form a complex with FIX. Thrombin is necessary to cleave FVIII upon which it loses its affinity for VWF. When FVIIIa is in solution, it can bind to phospholipids ${ }^{17}$. Unlike the activation of FV by meizothrombin, the activation of FVII requires thrombin in 
solution, that, unlike meizothrombin, is at risk of being cleared by antithrombin (AT), particularly in the presence of heparin.

The combination of both pro- and anticoagulant characteristics of FXa and FVa, ensures that coagulation takes place where it is necessary: on damaged cells and platelets where the procoagulant membranes are found. The spatial delineation of coagulation is further aided by TM, which is expressed on the surface of intact endothelial cells ${ }^{25}$. Thrombin that is bound to TM is able to cleave protein C (PC) to its activated form (APC), and due to this action thrombin loses its procoagulant properties $^{26}$.

APC is a strong inhibitory protein that, with the help of protein $\mathrm{S}$ and $\mathrm{FV}^{27,28}$, is able to degrade FVa and FVIIIa which are bound to procoagulant phospholipid surfaces $^{27,29}$. When the procoagulant surface is provided by platelets APC is not able to effectively cleave $\mathrm{FVa}^{30}$, in fact platelets seem to protect $\mathrm{FVa}$ from cleavage by $\mathrm{APC}^{31}$. This specificity has led to the believe that APC is not necessarily responsible for the delimitation of thrombin formation but rather that it is responsible for the prevention of clotting on intact vasculature ${ }^{32}$. Compared to APC protein $S$ has a greater inhibitory potential, as it is able to inhibit the prothrombinase complex on endothelial cells as well as on a platelet surface ${ }^{33}$.

APC is cleared from the bloodstream by the serine protease inhibitor (serpin) protein $\mathrm{C}$ inhibitor $(\mathrm{PCl}) . \mathrm{PCl}$ is a very indiscriminative inhibitor which has in fact a higher affinity for other coagulation factors e.g. FXa, thrombin and FVIla-TF than for $\mathrm{APC}^{34}$.

Fibrin was initially named antithrombin $1^{35}$ because it binds thrombin strongly. It has been shown that fibrin(ogen) is primarily a protector of thrombin activity, because of the limited access of $\alpha_{2}$ macroglobulin ( $\left.\alpha 2-M\right)$ to thrombin that is adsorbed to fibrin ${ }^{36}$. The most obvious antithrombin action of fibrin and probably the most teleological "reason" that a clot forms as soon as a trace of thrombin is formed, is that the clot is a spatial delineator. The structure of the clot prevents thrombin being washed from where it is most needed, or to spread in free solution where it can work disasters ${ }^{37}$

The blood platelets play a major role in coagulation in vivo. Due to the complexity of their role, this will not be described in detail here. For a comprehensive review, please refer to the subchapter on platelets in Versteeg et al. ${ }^{32}$. In brief, the role of platelets in coagulation starts when endothelium damage occurs. Upon damage, platelets are triggered to adhere to the wound and to each other. Several mechanisms are responsible for the increased platelet adhesiveness, amongst others: shape change, clustering of receptors and increased receptor expression ${ }^{32}$. Aggregating platelets also actively participate in coagulation. From the moment 
they are triggered to aggregate, some platelets are activated and start to express phosphatidylserine on their surface ${ }^{38,39}$, thereby providing a procoagulant surface. As was explained earlier, many coagulation proteins bind to such procoagulant phospholipid membrane layers where they react with other coagulation proteins. However, the existence of non-obstructive thrombi in large vessels is an indication that the forming plug, at some point, loses its propagating capabilities. This retardation of the growth of the plug is a phenomenon that is still poorly understood.

Platelets and the coagulation system are closely intertwined and their functions are not those of two separate processes. The group of Bruce and Barbara Furie showed that the formation of fibrin takes place within $15 \mathrm{~s}$ after a lesion is made in the vessel wall ${ }^{40}$. This rapid formation of fibrin indicates that thrombin must have been present almost at the onset of the formation of the platelet aggregate. This is further exemplified by the fact that it takes platelet poor plasma between 12-15s to clot at optimal TF concentration. Wherever there is fibrin, thrombin must have been present at $\sim 15$ s before its appearance to provide the feedback reactions for coagulation to accelerate. Their exact role is still the subject of debate, but it has been shown that microparticles do play a role in coagulation as well ${ }^{41}$. It has been argued that they adhere to a site of lesion before platelets and they are instrumental in generating thrombin ${ }^{42}$.

Further undermining the paradigm of primary haemostasis by platelets the bolstering of a primary plug by thrombin reinforces the idea that platelet aggregation is one voice in a concert of interactions involving plasma proteins and structured elements. Moreover, platelets play a vital role in the localization of coagulation at the site of injury. The confinement of the platelet aggregate reduces molecular transport rates within its core. This increases the concentration of soluble agonists which accelerate thrombin formation and subsequent platelet activation. Local accumulation of thrombin and the regulation of the density of platelet packing both contribute to thrombus growth. Moreover, the higher transport rates in the more permeable outer shell of the aggregate facilitate clearance and limit thrombin formation ${ }^{43}$.

The large amount of thrombin localized in the clot activates thrombin-activatable fibrinolysis inhibitor (TAFI) ${ }^{26}$. TAFI protects degradation of fibrin by cleavage of the C-terminal residue, thereby protecting fibrin from degradation by plasmin ${ }^{27}$. This feedback mechanism leads to the formation of a specific clot morphology, that is dependent on the amount of thrombin in the clot. A lot of thrombin gives a dense network of thin fibrin fibres, whereas a small amount of thrombin gives a coarse network of thick fibres ${ }^{46,47}$. 
Given the efficiency of all of these procoagulant processes it is not hard to imagine that there are counteracting forces present as well. Several have been mentioned already, but the main custodians are the antithrombins. The antithrombins present themselves to thrombin as a substrate to be cleaved, but after doing so thrombin finds itself to be stuck and incapable of further action ${ }^{35,48}$. The most important of the antithrombins was traditionally called antithrombin III (ATIII), and is responsible for clearance of about $2 / 3$ of the total amount of thrombin ${ }^{49}$. The 2 nd most important antithrombin is $\alpha 2-\mathrm{M}$ that clears about $1 / 4$ of the total amount of thrombin ${ }^{50}$, the remaining fraction is cleared by miscellaneous inhibitors. Because there are so many antithrombins, we hesitate to follow the official nomenclature and will speak of "antithrombin III" when referring to the main antithrombin.

The efficiency of ATIII is enhanced a great deal by heparin (a common anticoagulant drug). Heparins are glycosaminoglycans that consist of chains of alternating residues of d-glucosamine and uronic acid, glucuronic acid or iduronic acid $^{51}$. Along the chain of saccharides, every 50-100 sugar units a specific sequence of 5 saccharide units occurs that has the ability to strongly bind ATIII ${ }^{52}$, which brings about a conformational change in ATIII that enhances its activity. Thrombin binds loosely anywhere to the saccharide chain without the need for a specific saccharide sequence. It moves quickly along the chain by lateral diffusion and so will meet-up with ATIII more easily, so much more easily in fact that heparin can increase the inhibition rate a thousand fold ${ }^{53}$.

\section{Measuring the coagulation system}

A concise overview of the coagulation tests used in the clinic is given by Bates and Weit $z^{54}$. Although the paper was published in 2005, the text is still very representative of the current situation in the clinical coagulation laboratory. The overview below gives a concise summary of the work of Bates with a few additions in the case of newly implemented test. The main aim of the representation of the tests below is to stress that these tests and their results do not represent the entirety of the coagulation system.

Most coagulation tests are defective approximations of a (specific) part/function of the coagulation system. They rather emphasize the initiation mechanism instead of the integral system of thrombin production. When rigorously standardized a clotting time, like the prothrombin time (PT) can be quite useful, e.g. in the monitoring of anticoagulant therapy with vitamin $\mathrm{K}$ antagonists ${ }^{55}$. The activated partial thromboplastin time (aPTT) defies all standardisation efforts ${ }^{56}$ and is unsuitable for the monitoring of heparin therapy ${ }^{57}$. 
It is their representation of the initiation phase that makes clotting times inept for testing conditions that depend on the production phase of thrombin generation, i.e. thrombotic tendency or bleeding tendency dependent upon the amount of thrombin formed. In order to represent the entirety of the complex system, a test is required that goes beyond the initiation phase. That the amount of thrombin is important follows e.g. from the thrombotic tendency that occurs when the protein $S$ dependent system fails (by the use of oral anticonception or factor $V$ Leiden). Why the amount of thrombin is important may be due to various causes, such as e.g. insufficient defence against fibrinolysis through lack of TAFI activation.

Despite the specific nature of the tests described below, they are at times applied for the detection of other pathologies than those described here. It is not impossible to do so, but it has to be understood that value of the results of an assay that measures the starting mechanism of coagulation only, is very limited in describing the complexity of a system as a whole. Most of the current coagulation tests operate in the same general principle as depicted with the APTT below. The variations can be found in the contents of the test tube with differing reagents or by the use of whole blood or blood plasma.

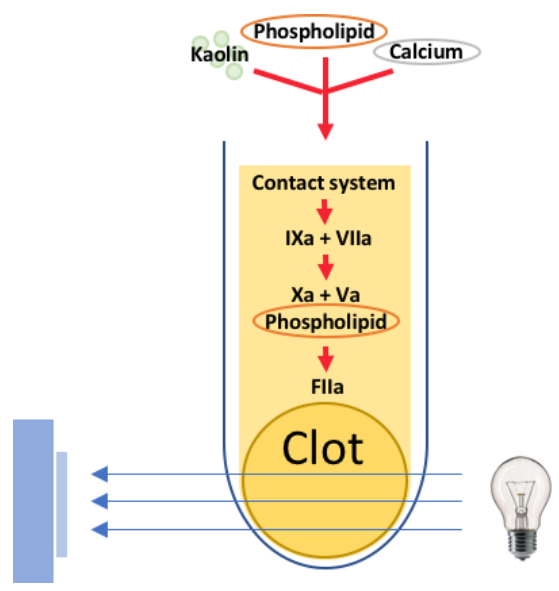

Figure: 1 Activated partial thromboplastin time (APTT).

Sample used: Citrated PPP, Activator: FXII activator e.g., kaolin, ellagic acid, celite or silica + diluted phospholipids (cephalin) and calcium, Used for: FVIII, FIX, FXI, FXII, HK, prekallikrein, FX, FV, FII, and/or fibrinogen deficiencies and monitoring of intravenous unfractionated heparin.

As can be observed in figure 1, a trigger (in this case a mixture of phospholipids, calcium and kaolin) is added to platelet poor plasma. This sets in motion a series of reactions that ultimately lead to the formation of a clot. A photo sensitive optic, that is able to record the disturbance in the light detects the clot at which moment 
the test stops and the time is recorded. As noted before, the tests described below are all clotting time tests and a apply a similar principle but differ in their trigger as well as reaction substrate.

- Prothrombin time (PT), Sample used: Citrated PPP, Activator: Recombinant or purified TF Used for: FII, FV, FVII, FX and fibrinogen deficiencies ${ }^{5,58}$. Vitamin $\mathrm{K}$ def. and fibrin degradation products. Warfarin, acenocoumarol, and fenprocoumon therapy through the INR.

- Activated clotting time (ACT), Sample used: Whole blood Activator: Kaolin, celite or glass particles Used for: High dose heparin treatment or bivalirudin treatment. Notice: besides systems using optical detection, devices that apply electro-chemical detection of thrombin cleaving an electro-chemical substrate are available on the market as well ${ }^{59}$.

- Thrombin clotting time (TCT), Sample used: Citrated PPP Activator: Thrombin Used for: Low fibrinogen, dysfibrinogenemia or elevated fibrinogen degradation products.

- Ecarin clotting time (ECT), Sample used: Citrated PPP Activator: Purified meizo-thrombin activator from the venom of the Russels viper (Echis Carinatus) Used for: Treatment with direct thrombin inhibitors ${ }^{60}$.

Apart from these clotting time tests, there are other specific coagulation test that are currently use. Most of these apply different methods of detection making it difficult to cluster them. The "other tests" are usually methods to determine a single element (factor) of the clotting system (Fibrinogen or factors VIII or IX for the management of haemophilia), tests designed for measuring specific anticoagulant treatment (e.g. anti-factor Xa activity for heparins) or indicators of ongoing coagulation (D-dimers, TAT-complexes). They will not be further discussed here.

Most tests that are being used in the clinic have faced the challenge of standardisation. Standardizing tests is crucial to get consistent results but has proven to be difficult for any clinical test and coagulation tests are no exception to this rule. One of the main contributors to the high inter-laboratory variability, of what can be considered a "simple" coagulation test like e.g. a clotting time, has been the composition of the reagents. In an attempt to standardize the PT the International Normalized Ratio (INR) has been established by the world health organization $(\mathrm{WHO})^{61}$. Without this effort of standardization, PT results using different reagents cannot be compared ${ }^{62}$. However, despite the stringent standards, unreliable reporting of the international sensitivity index (ISI) by thromboplastin manufacturers ${ }^{63}$ and the variation in precision of INR determination as a result of different reagent and coagulometer combinations still causes variation of the results between laboratories. 
Most research laboratories, do not have to abide to the stringent clinical requirements. Therefore, researchers have much more tools at its disposal to measure the coagulation system. Coagulation tests in the research laboratory range from specific test for platelet function to rheology or flow measurements. The present work however is restricted to the thrombin generation test. Thrombin being responsible for the "final" step of the coagulation system with the cleavage of fibrinogen to fibrin is dependent upon the interactions of the preceding proteins for its formation. Therefore, measuring thrombin formation over time gives an indication of the functioning of the system as a whole, way beyond the formation of a clot which already appears when only $5 \%$ of all thrombin is formed. This broader view generated by the TG test gives it the potential to provide qualitative and quantitative information about the coagulation system as a whole.

Throughout the last decades considerable evidence has been published that the amount of thrombin activity that develops in a sample of clotting blood(-plasma) is an indicator of the risk of bleeding ${ }^{64-67}$ as well as venous thrombosis ${ }^{68-76}$. Moreover, it relates to the risk of arterial thrombosis, be it in a less straightforward manner ${ }^{77-79}$. It is a common denominator of the effect of all anticoagulants ${ }^{80-87}$ and, when it is measured in platelet rich plasma, it is inhibited by platelet inhibitors ${ }^{88,89}$. Therefore, it has a promising outlook for use as a clinically useful parameter.

Similarly to the more basic clotting time based assays, the Calibrated Automated Thrombin Generation test (CAT-TG) ${ }^{3}$ that has been used in research laboratories for over 15 years now, has its share of difficulties with respect to standardization and reproducibility of the results between different laboratories.

This poor inter-laboratory variability is considered to be the main issue preventing broad clinical implementation of TG-test ${ }^{6}$. The high variability in test results between laboratories has been attributed to e.g. a lack of automation and its sensitivity to pre-analytical variables ${ }^{90}$. Rigorous standardization of the preanalytical and analytical procedure and normalization relative to a reference plasma indeed improved the $\mathrm{CV}^{91,92}$. Moreover, a multicentre study from Frenchspeaking countries (France, Belgium, Switzerland) carried out under "real life" conditions in clinical and research laboratories showed that the use of a common reference plasma could improve inter-laboratory variability of most TG parameters except in cases of frank hypo-coagulability ${ }^{93}$. These efforts have as yet not added up to a test which is sufficiently robust to warrant broad acceptation in the clinical laboratory.

\section{Measuring thrombin generation}


Thrombin generation (TG) was first described in 1953 by MacFarlane and Biggs as a whole blood test ${ }^{94}$. In the same edition of the same journal Pitney and Dacie described the test in recalcified plasma ${ }^{95}$. Both methods were triggered by adding preheated calcium to a sample of clotting blood or plasma, out of which, individual timed (sub)samples were then taken at regular intervals. The subsample then is transferred to a test-tube with a fibrinogen preparation and the clotting time is recorded. The relation between clotting time and thrombin concentration has been previously established. From the observed clotting times the formation and subsequent decay of thrombin in these samples is then calculated. Despite its labour intensiveness this TG test, sometimes in a personalized version, has proved itself useful in advancing research in e.g. haemophilia ${ }^{96,97}$.

Before TG could be more widely adopted, the test had to be fundamentally altered. In the present situation of the clinical chemistry lab, where laboratory work is reduced to putting samples in an automaton and waiting for the figures that it prints out - in this ambiance anything looking remotely as a subsampling experiment is an anachronism. A fundamentally new approach was required. This came in three concurrent steps. The first was from S. Béguin who added a chromogenic thrombin substrate directly to the clotting plasma. The second from C. Hemker, who realized that, if this were to reflect the complete thrombin generation curve, the substrate used should be so poorly reactive to thrombin that it should not be consumed during the course of the thrombin generation, and the third from R. Wagenvoord who suggested that - in order to be able to know what reaction velocity reflects what thrombin concentration, a calibration experiment had to be run in parallel. The combination of these three ideas allowed continuous measurement of thrombin formation ${ }^{98}$. In this way, through the detection of the reaction product that is cleaved from this substrate by thrombin, the formation and subsequent decay of thrombin in a plasma sample can be measured over time. Because the formation of fibrin disturbs the detection of the reaction product, the plasma has to be defibrinated prior to the measurement, or a fibrin polymerization inhibitor had to be added.

Fibrin, however, is an essential part of the thrombin generation mechanism ${ }^{36}$ so it was an essential step forward when the fluorogenic substrates brought an end to this inconvenience. This led to the presentation of Calibrated Automated Thrombography (CAT) ${ }^{3}$.

The idea of using a fluorogenic substrate not being patented it could be followed in the Technothrombin TGA method ${ }^{99}$. The simultaneous calibration through a parallel experiment had been patented, however, so that accurate calibration in the latter method will have to wait for the patent to expire. 
The use of both chromogenic and fluorogenic substrates has led to a bifurcation in the commercially available thrombin generation assays. Currently there are three automated TG assays available, two that use the fluorogenic substrate: ST-Genesia by Diagnostica Stago, Inc. (Asnieres sur Seine, France), the CEVERON alpha TGA by Technoclone Herstellung von Diagnostika und Arzneimitteln GmbH (Vienna, Austria) and the Behring Coagulation System (BCS) Siemens Healthcare GmbH (Erlangen, Germany), using a chromogenic substrate.

Because of the important role of fibrin in physiological thrombin generation we restricted our research to methods using a fluorogenic substrate. The article by Kintigh et $\mathrm{al}^{99}$ is referred to for a comparison between the three automated assays. Besides the three automated methods for TG, four semi-automated TG assays exist. The same bifurcation as with the automated methods persists here with two assays using a chromogenic substrate and two using a fluorogenic substrate. The assays using a fluorogenic substrate can be seen as predecessors of the ST-Genesia and the Ceveron alpha TGA, as this method of TG is the further focus of this work they will be elaborately discussed below.

But first, a brief review of the two assays that make use of the chromogenic substrate: HemoScan Thrombin Generation Assay (HemoScan, Groningen, The Netherlands), is a kit that is intended to measure the influence of biomaterials on thrombin generation. A plasma sample is exposed to the biomaterial to be investigated, where after the TG results with and without exposure are compared. The method utilizes subsampling, rather than continuous detection. After the sample is taken from the reaction mixture, the reaction is stopped and the formed thrombin is left to react with a chromogenic substrate. The thrombin concentrations in the subsamples are subsequently determined from a calibration curve. The HemoScan assay serves its purpose but is not intended for diagnostic use.

Pefakit in-TDT (Pentapharm, Aesch, Switzerland), triggers thrombin generation by contact activation. The cleavage of a chromogenic substrate is subsequently detected over time by measuring the optical density of the reaction mixture. A fibrin polymerization inhibitor is added so as to prevent the formation of a fibrin clot and the related optical disturbance of the measurement. After taking the first derivative of the data this method determines two parameters: the lag-time and the peak. The results are not calibrated against a standard concentration of thrombin, rather the values for the patient sample are expressed as a percentage of normal. 
The CAT assay measures thrombin generation in a sample of platelet poor plasma (PPP) or platelet rich plasma (PRP) $)^{3}$ and has been further developed to be used in whole blood ${ }^{100}$. The TG experiment is based on the detection of the reaction product of a ZGGR-AMC substrate by thrombin. The amount of thrombin in the sample in which thrombin is generating (the staple sample), is calculated by comparison to the fluorescence that develops in a separate sample of the same plasma in which a constant known amount of thrombin activity converts the substrate (the calibrator sample). In this way, the value of the calibration factor can be used to calculate the concentration of thrombin. How this is done and how it should be done is the main subject of this thesis. Figure 1a displays a typical fluorescence trace of such experiment. The speed with which thrombin is formed over time (i.e. the first derivative of the original curve) gives a rough approximation of the thrombin generation curve (figure $1 \mathrm{~b}$ ) and allows to distinguish the separate phases of the process: the lag-time (1), the burst of thrombin formation (2) and thrombin decay (3). It can also be noticed is that the curve does not return to the $x$-axis due to residual thrombin activity of the $\alpha 2$-macroglobulin thrombin $(\alpha 2 \mathrm{M}-\mathrm{T}$ ) complex. Thrombin entrapped in the $\alpha 2$ macroglobulin molecule loses all known physiological activity but still can split small substrates. This phenomenon and how to disentangle it from real thrombin activity will be discussed in more detail in following chapters.

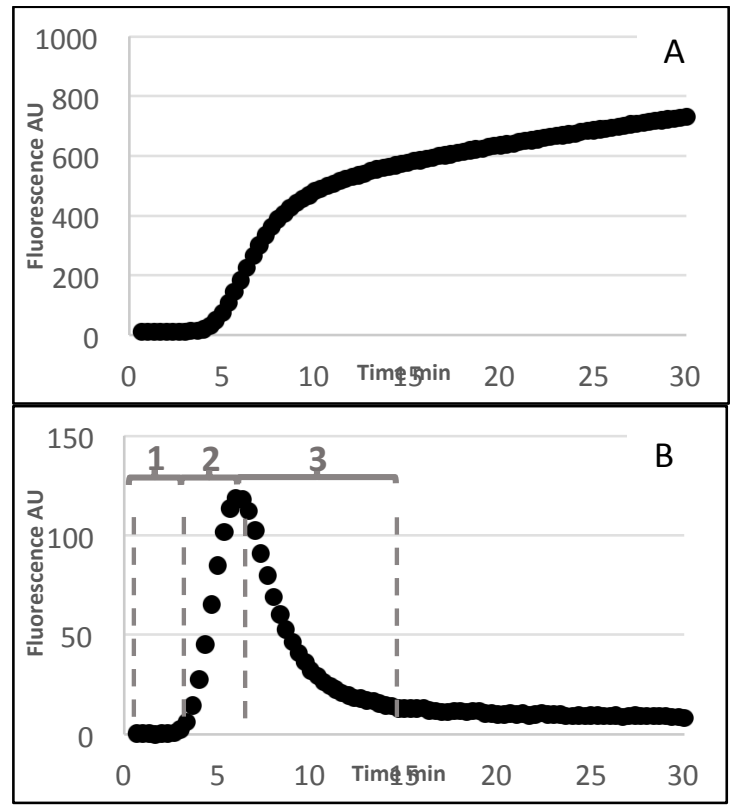

Figure 2 thrombin generation data.

A, "raw" fluorescence trace of thrombin generation. B, First derivative of A with lag-time " 1 ", thrombin formation " 2 ", and thrombin decay " 3 ". 
The reaction velocity during the experiment is not determined by the concentration of thrombin (and $(\alpha 2 \mathrm{M}-\mathrm{T}$ ) alone, also the concentration of substrate plays a role here. Because during the reaction the substrate is consumed the same amount of thrombin will not cause the same reaction velocity in the beginning of the experiment and later on.

Also, the relation between fluorescence and concentration of product is not linear. The consequences of substrate consumption and inner filter effect and how to correct for them will be discussed in much detail in the following chapters. Here we only stress the unique feature of the CAT-method, i.e. that for each TGexperiment there is a parallel calibration experiment in which, in the same plasma, the same amount of substrate is converted by a known fixed amount of thrombin activity (in the form of the $(\alpha 2 \mathrm{M}-\mathrm{T} \text { complex })^{101}$. This provides a sample-specific calibration factor at every level of fluorescence and therefore in principle allows correct determination of thrombin concentrations. How to carry out such calculations and searching for alternative but equally correct methods of calibration is the main subject of this thesis. 


\section{References}

1. Zwaginga, J. J. et al. Flow-based assays for global assessment of hemostasis. Part 2: current methods and considerations for the future. Journal of Thrombosis and Haemostasis 4, 2716-2717 (2006).

2. Ignjatovic, V. et al. Differences in the mechanism of blood clot formation and nanostructure in infants and children compared with adults. Thrombosis Research 136, 1303-1309

3. Hemker, H. C. et al. Calibrated Automated Thrombin Generation Measurement in Clotting Plasma. Pathophysiol Haemos Thromb 33, 4-15 (2003).

4. Hemker, H. C., Giesen, P. L., Ramjee, M., Wagenvoord, R. \& Béguin, S. The thrombogram: monitoring thrombin generation in platelet-rich plasma. Thromb. Haemost. 83, 589-591 (2000).

5. Jain, A. et al. Assessment of whole blood thrombosis in a microfluidic device lined by fixed human endothelium. Biomed Microdevices 18, 73 (2016).

6. Dargaud, Y. et al. Effect of standardization and normalization on imprecision of calibrated automated thrombography: an international multicentre study. British Journal of Haematology 139, 303-309 (2007).

7. Veith, I. The yellow emperor's classic of internal medicine. (Univ of California Press, 1949).

8. Bodnár, T., Galdi, G. \& Nečasová, Š. Fluid-Structure Interaction and Biomedical Applications. (2014). doi:10.1007/978-3-0348-0822-4

9. Crawley, J., Zanardelli, S., Chion, C. \& Lane, D. The central role of thrombin in hemostasis. Journal of thrombosis and haemostasis 5, 95-101 (2007).

10. Banfield, D. K., Irwin, D. M., Walz, D. A. \& MacGillivray, R. T. Evolution of prothrombin: isolation and characterization of the cDNAs encoding chicken and hagfish prothrombin. Journal of Molecular Evolution 38, 177-187 (1994).

11. Downing, M. R., Butkowski, R. J., Clark, M. M. \& Mann, K. G. Human prothrombin activation. Journal of Biological Chemistry 250, 8897-8906 (1975).

12. Szotowski, B., Antoniak, S., Poller, W., Schultheiss, H.-P. \& Rauch, U. Procoagulant Soluble Tissue Factor Is Released From Endothelial Cells in Response to Inflammatory Cytokines. Circulation Research 96, 1233-1239 (2005).

13. Eigenbrot, C. et al. The factor VII zymogen structure reveals reregistration of $\beta$ strands during activation. Structure 9, 627-636 (2001).

14. Bajaj, S. P., Rapaport, S. I. \& Brown, S. F. Isolation and characterization of human factor VII. Activation of factor VII by factor Xa. Journal of Biological Chemistry 256, 253-259 (1981).

15. Krishnaswamy, S., Mann, K. \& Nesheim, M. The prothrombinase-catalyzed activation of prothrombin proceeds through the intermediate meizothrombin in an ordered, sequential reaction. Journal of Biological Chemistry 261, 8977-8984 (1986).

16. Hemker HC. Formation of prothrombin converting activity. Nature 215, 248 (1967).

17. Rosing, J., Tans, G., Govers-Riemslag, J., Zwaal, R. \& Hemker, H. C. The role of phospholipids and factor $V a$ in the prothrombinase complex. Journal of Biological Chemistry 255, 274-283 (1980).

18. Nesheim, M. E., Taswell, J. B. \& Mann, K. The contribution of bovine Factor $V$ and Factor Va to the activity of prothrombinase. Journal of Biological Chemistry 254, 10952-10962 (1979).

19. Østerud, B. Tissue factor/TFPI and blood cells. Thrombosis research 129, 274-278 (2012).

20. Sandset, P. M. Tissue factor pathway inhibitor (TFPI)-an update. Pathophysiology of Haemostasis and Thrombosis 26, 154-165 (1996).

21. Al Dieri, R., Bloemen, S., Kelchtermans, H., Wagenvoord, R. \& Hemker, H. A new regulatory function of activated factor $V$ : inhibition of the activation by tissue factor/factor VII (a) of factor X. Journal of Thrombosis and Haemostasis 11, 503-511 (2013). 
22. Josso, F. \& Prou-Wartelle, O. Interaction of tissue factor and factor VII at the earliest phase of coagulation. Thromb Diath Haemorrh Supp/17, 35-44 (1965).

23. Hemker HC. Reaction sequence of blood coagulation. Nature 215, 1201 (1967).

24. Xi, M., Béguin, S. \& Hemker, H. C. Importance of Factor-IX-Dependent Prothrombinase Formation - The Josso Pathway - in Clotting Plasma. Pathophysiology of Haemostasis and Thrombosis 19, 301-308 (1989).

25. Esmon, N. L., Owen, W. \& Esmon, C. Isolation of a membrane-bound cofactor for thrombincatalyzed activation of protein C. Journal of Biological Chemistry 257, 859-864 (1982). Esmon, C. T., Esmon, N. \& Harris, K. Complex formation between thrombin and thrombomodulin inhibits both thrombin-catalyzed fibrin formation and factor $\mathrm{V}$ activation. Journal of Biological Chemistry 257, 7944-7947 (1982).

27. DAHLBÄCK, B. \& STENFLO, J. Inhibitory Effect of Activated Protein C on Activation of Prothrombin by Platelet-Bound Factor Xa. European Journal of Biochemistry 107, 331-335 (1980).

28. Stenflo, J. \& Jönsson, M. Protein S, a new vitamin K-dependent protein from bovine plasma. FEBS letters 101, 377-381 (1979).

29. Dahlback, B. Factor $\mathrm{V}$ and protein $\mathrm{S}$ as cofactors to activated protein C. Haematologica 82, 91-95 (1997).

30. Oliver, J. A., Monroe, D. M., Church, F. C., Roberts, H. R. \& Hoffman, M. Activated protein C cleaves factor $V a$ more efficiently on endothelium than on platelet surfaces. Blood 100 , 539-546 (2002).

31. Camire, R. M., Kalafatis, M., Simioni, P., Girolami, A. \& Tracy, P. B. Platelet-derived factor $\mathrm{Va} / \mathrm{Va}$ Leiden cofactor activities are sustained on the surface of activated platelets despite the presence of activated protein C. Blood 91, 2818-2829 (1998).

32. Versteeg, H. H., Heemskerk, J. W., Levi, M. \& Reitsma, P. H. New fundamentals in hemostasis. Physiological reviews 93, 327-358 (2013).

33. Hackeng, T. M., Van't Veer, C., Meijers, J. \& Bouma, B. N. Human protein S inhibits prothrombinase complex activity on endothelial cells and platelets via direct interactions with factors Va and Xa. Journal of Biological Chemistry 269, 21051-21058 (1994).

34. Geiger, M., Wahlmüller, F. \& Furtmüller, M. The Serpin Family: Proteins with Multiple Functions in Health and Disease. (Springer, 2015).

35. Seegers, W. H., Yoshinari, M. \& Landaburu, R. Antithrombin as substrate for the enzyme thrombin. Thrombosis et diathesis haemorrhagica 4, 293-298 (1960).

36. Kremers, R. M. W., Wagenvoord, R. J. \& Hemker, H. C. The effect of fibrin(ogen) on thrombin generation and decay. Thromb Haemost 112, 486-494 (2014).

37. Baglin, T. Disseminated intravascular coagulation: diagnosis and treatment. BMJ 312, 683686 (1996).

38. Zwaal RFA. Membrane asymmetry and blood coagulation. Nature 268, 358 (1977).

39. Munnix, I. C. et al. Segregation of platelet aggregatory and procoagulant microdomains in thrombus formation. Arteriosclerosis, thrombosis, and vascular biology 27, 2484-2490 (2007).

40. Furie, B. \& Furie, B. C. Thrombus formation in vivo. The Journal of clinical investigation 115 , 3355-3362 (2005).

41. Ridger, V. C. et al. Microvesicles in vascular homeostasis and diseases. Position Paper of the European Society of Cardiology (ESC) Working Group on Atherosclerosis and Vascular Biology. Thromb. Haemost. 117, 1296-1316 (2017).

42. Giesen, P. L. A. et al. Blood-borne tissue factor: Another view of thrombosis. PNAS 96, 2311-2315 (1999).

43. Welsh, J. D. et al. A systems approach to hemostasis: 1. The interdependence of thrombus architecture and agonist movements in the gaps between platelets. Blood 124, 1808-1815 (2014). 
44. Nesheim, M. \& Bajzar, L. HISTORICAL SKETCH: The discovery of TAFI. Journal of Thrombosis and Haemostasis 3, 2139-2146 (2005)

45. Bajzar, L., Manuel, R. \& Nesheim, M. E. Purification and characterization of TAFI, a thrombin-activable fibrinolysis inhibitor. Journal of Biological Chemistry 270, 14477-14484 (1995).

46. Weisel, J. W. \& Nagaswami, C. Computer modeling of fibrin polymerization kinetics correlated with electron microscope and turbidity observations: clot structure and assembly are kinetically controlled. Biophysical journal 63, 111-128 (1992).

47. Ryan, E. A., Mockros, L. F., Weisel, J. W. \& Lorand, L. Structural origins of fibrin clot rheology. Biophysical journal 77, 2813-2826 (1999).

48. Abildgaard, U. Inhibition of the Thrombin-Fibrinogen Reaction by Heparin and Purified Cofactor. European Journal of Haematology 5, 440-453 (1968).

49. Beguin, S., Kessels, H., Dol, F. \& Hemker, H. The consumption of antithrombin III during coagulation, its consequences for the calculation of prothrombinase activity and the standardisation of heparin activity. Thrombosis and haemostasis 68, 136-142 (1992).

50. Steinbuch, M., Blatrix, C. \& Josso, F. Antiproteinase activity of alpha-2-macroglobulin. II. Its role in progressive antithrombin states. Revue francaise d'etudes cliniques et biologiques 13, 179-186 (1968).

51. Weitz, J. I. Low-Molecular-Weight Heparins. New England Journal of Medicine 337, 688699 (1997).

52. Choay, J. et al. Structure-activity relationship in heparin: a synthetic pentasaccharide with high affinity for antithrombin III and eliciting high anti-factor Xa activity. Biochemical and biophysical research communications 116, 492-499 (1983).

53. Wagenvoord R. Linear diffusion of thrombin and factor Xa along the heparin molecule explains the effects of extended heparin chain lengths. Thrombosis Research 122, 237 (2008).

54. Bates, S. M. \& Weitz, J. I. Coagulation Assays. Circulation 112, e53-e60 (2005).

55. Ansell, J. et al. Managing Oral Anticoagulant Therapy. CHEST 119, 22S-38S (2001).

56. Eikelboom, J. W. \& Hirsh, J. Monitoring unfractionated heparin with the aPTT: Time for a fresh look. Thromb Haemost 96, 547-552 (2006).

57. al Dieri, R., Alban, S., Béguin, S. \& Hemker, H. C. Thrombin generation for the control of heparin treatment, comparison with the activated partial thromboplastin time. J. Thromb. Haemost. 2, 1395-1401 (2004).

58. Fowler, A. \& Perry, D. J. Laboratory monitoring of haemostasis. Anaesthesia 70, 68-72 (2015).

59. Martin, C. L. i-STAT - Combining Chemistry and Haematology in PoCT. Clin Biochem Rev 31, 81-84 (2010)

60. Lange, U., Nowak, G. \& Bucha, E. Ecarin Chromogenic Assay - A New Method for Quantitative Determination of Direct Thrombin Inhibitors Like Hirudin. Pathophysiology of Haemostasis and Thrombosis 33, 184-191 (2003).

61. Kirkwood, T. B. Calibration of reference thromboplastins and standardisation of the prothrombin time ratio. Thromb Haemost 49, 238-244 (1983).

62. Zucker, S., Cathey, M. H., Sox, P. J. \& Hall, E. C. Standardization of Laboratory Tests for Controlling Anticoagulant Therapy. Am J Clin Pathol53, 348-354 (1970).

63. Hirsh, J. Oral Anticoagulant Drugs. New England Journal of Medicine 324, 1865-1875 (1991).

64. Dargaud, Y., Lienhart, A. \& Negrier, C. Prospective assessment of thrombin generation test for dose monitoring of bypassing therapy in hemophilia patients with inhibitors undergoing elective surgery. Blood 116, 5734-5737 (2010).

65. Millet, A. et al. Thrombin generation in patients with acquired haemophilia and clinical bleeding risk. Br. J. Haematol. 153, 136-139 (2011). 
66. Bosch, Y. P. J. et al. Measurement of thrombin generation intra-operatively and its association with bleeding tendency after cardiac surgery. Thromb. Res. 133, 488-494 (2014).

67. Bloemen, S., Zwaveling, S., Ten Cate, H., Ten Cate-Hoek, A. \& de Laat, B. Prediction of bleeding risk in patients taking vitamin $\mathrm{K}$ antagonists using thrombin generation testing. PLOS ONE 12, e0176967 (2017).

68. Tans, G. et al. Activated protein $\mathrm{C}$ resistance determined with a thrombin generation-based test predicts for venous thrombosis in men and women. Br. J. Haematol. 122, 465-470 (2003).

69. Hron, G., Kollars, M., Binder, B. R., Eichinger, S. \& Kyrle, P. A. Identification of patients at low risk for recurrent venous thromboembolism by measuring thrombin generation. JAMA 296, 397-402 (2006).

70. Buyue, Y., Whinna, H. C. \& Sheehan, J. P. The heparin-binding exosite of factor IXa is a critical regulator of plasma thrombin generation and venous thrombosis. Blood 112, 32343241 (2008).

71. Sanchez, C., Alessi, M. C., Saut, N., Aillaud, M. F. \& Morange, P. E. Relation between the antithrombin Cambridge II mutation, the risk of venous thrombosis, and the endogenous thrombin generation. J. Thromb. Haemost. 6, 1975-1977 (2008).

72. Lavigne-Lissalde, G. et al. Prothrombin G20210A carriers the genetic mutation and a history of venous thrombosis contributes to thrombin generation independently of factor II plasma levels. J. Thromb. Haemost. 8, 942-949 (2010).

73. Ay, C. et al. Prediction of venous thromboembolism in patients with cancer by measuring thrombin generation: results from the Vienna Cancer and Thrombosis Study. J. Clin. Oncol. 29, 2099-2103 (2011)

74. Anžej Doma, S., Vučnik, M., Božič Mijovski, M., Peternel, P. \& Stegnar, M. Enhanced thrombin generation in women with a history of oral contraception-related venous thrombosis. Thromb. Res. 132, 621-626 (2013).

75. Hylckama Vlieg, A. et al. The risk of a first and a recurrent venous thrombosis associated with an elevated D-dimer level and an elevated thrombin potential: results of the THE-VTE study. Journal of Thrombosis and Haemostasis 13, 1642-1652 (2015).

76. Martin-Fernandez, L. et al. Genetic Determinants of Thrombin Generation and Their Relation to Venous Thrombosis: Results from the GAIT-2 Project. PLOS ONE 11, e0146922 (2016).

77. Maurer, E. et al. Targeting Platelet GPIbß Reduces Platelet Adhesion, GPIb Signaling and Thrombin Generation and Prevents Arterial ThrombosisSignificance. Arteriosclerosis, Thrombosis, and Vascular Biology 33, 1221-1229 (2013).

78. Ten Cate, H. \& Hemker, H. C. Thrombin Generation and Atherothrombosis: What Does the Evidence Indicate? J Am Heart Assoc 5, (2016).

79. Tosi, F. et al. Increased plasma thrombin potential is associated with stable coronary artery disease: An angiographically-controlled study. Thromb. Res. 155, 16-22 (2017).

80. Nagashima, H. Studies on the different modes of action of the anticoagulant protease inhibitors DX-9065a and Argatroban. I. Effects on thrombin generation. J. Biol. Chem. 277, 50439-50444 (2002).

81. Walker, C. P. R. \& Royston, D. Thrombin generation and its inhibition: a review of the scientific basis and mechanism of action of anticoagulant therapies. Br J Anaesth 88, 848863 (2002).

82. Brocal, I., Marco, P., Lucas, J., Verdú, J. \& Tarín, F. Thrombin generation test in patients under anticoagulant therapy with vitamin K antagonists. Thromb. Haemost. 101, 594-595 (2009). 
83. Robert, S. et al. Is thrombin generation the new rapid, reliable and relevant pharmacological tool for the development of anticoagulant drugs? Pharmacol. Res. 59, 160-166 (2009).

84. Bloemen, S., Hemker, H. C. \& Al Dieri, R. Large inter-individual variation of the pharmacodynamic effect of anticoagulant drugs on thrombin generation. Haematologica 98, 549-554 (2013).

85. Chowdary, P. et al. Thrombin generation assay identifies individual variability in responses to low molecular weight heparin in pregnancy: implications for anticoagulant monitoring. Br. J. Haematol. 168, 719-727 (2015).

86. Tripodi, A. et al. How the direct oral anticoagulant apixaban affects thrombin generation parameters. Thromb. Res. 135, 1186-1190 (2015).

87. D'Alessio, A. et al. Long Term Low Molecular Weight Heparin Anticoagulant Therapy Modulates Thrombin Generation and D-dimer in Patients with Cancer and Venous Thromboembolism. Cancer Invest. 35, 490-499 (2017).

88. Reverter, J. C. et al. Inhibition of platelet-mediated, tissue factor-induced thrombin generation by the mouse/human chimeric 7E3 antibody. Potential implications for the effect of c7E3 Fab treatment on acute thrombosis and 'clinical restenosis'. J. Clin. Invest. 98, 863-874 (1996).

89. Keularts, I. M., Béguin, S., de Zwaan, C. \& Hemker, H. C. Treatment with a GPIIb/IIla antagonist inhibits thrombin generation in platelet rich plasma from patients. Thromb. Haemost. 80, 370-371 (1998).

90. Loeffen, R. et al. Preanalytic variables of thrombin generation: towards a standard procedure and validation of the method. J. Thromb. Haemost. 10, 2544-2554 (2012).

91. Dargaud, Y. et al. Standardisation of thrombin generation test - which reference plasma for TGT?: An international multicentre study. Thrombosis Research 125, 353-356 (2010).

92. Dargaud, Y. et al. Proposal for standardized preanalytical and analytical conditions for measuring thrombin generation in hemophilia: communication from the SSC of the ISTH. J Thromb Haemost 15, 1704-1707 (2017).

93. Perrin, J. et al. Large external quality assessment survey on thrombin generation with CAT: further evidence for the usefulness of normalisation with an external reference plasma. Thrombosis Research 136, 125-130 (2015).

94. Macfarlane, R. G. \& Biggs, R. A Thrombin Generation Test. J Clin Pathol 6, 3-8 (1953)

95. Pitney, W. R. \& Dacie, J. V. A Simple Method of Studying the Generation of Thrombin in Recalcified Plasma. J Clin Pathol 6, 9-14 (1953).

96. Sjølin, K. E. Deficiency of the Hageman factor demonstrated by the thrombin generation test. Thromb Diath Haemorrh 1, 153-157 (1957).

97. Sjølin, K.-E. The Thrombin Generation Test in the Diagnosis of Classical Hemophilia and Christmas Disease. Scandinavian Journal of Clinical and Laboratory Investigation 8, 138-144 (1956).

98. Hemker, H. C., Wielders, S., Kessels, H. \& Béguin, S. Continuous registration of thrombin generation in plasma, its use for the determination of the thrombin potential. Thromb Haemost 70, 617-624 (1993).

99. Kintigh, J., Monagle, P. \& Ignjatovic, V. A review of commercially available thrombin generation assays. Research and Practice in Thrombosis and Haemostasis 2, 42-48 (2018).

100. Ninivaggi, M. et al. Whole-Blood Thrombin Generation Monitored with a Calibrated Automated Thrombogram-Based Assay. Clinical Chemistry 58, 1252-1259 (2012).

101. De Smedt, E. et al. The technique of measuring thrombin generation with fluorogenic substrates: 1. Necessity of adequate calibration. Thromb Haemost 100, 343-9 (2008) 

Chapter 2 Near Patient Thrombin Generation in Patients

\section{Undergoing Elective Cardiac Surgery}

Martijn Moorlag", Evelien Schurgers", Ganeshram Krishnamoorthy, Anne Bouwhuis, Theo Lindhout, Hilde Kelchtermans, Marcus D. Lance, Bas de Laat

9, both authors contributed equally to this work 


\section{Abstract}

Background Measuring thrombin generation (TG) in plasma increasingly gained attention as a diagnostic tool in the field of thrombosis and haemostasis. To include the contribution of all blood cells, recently the whole blood TG method was developed.

Methods We changed the calculation method of the standard calibrated automated thrombography (CAT) to a method only considering the data until the peak of TG, thereby considerably reducing the time from blood draw to result. By redesigning the method, the blood volume per test was reduced to $15 \mu \mathrm{l}$.

Results For all TG parameters the inter-assay variation proved to be below $15 \%$. The inter-individual variation of all parameters was comparable to the CAT method. 33 patients undergoing cardiothoracic surgery were included to investigate whether our assay correlates with post-operative blood loss. Upon dividing patients into severe and mild bleeders, significant differences between both groups were found for the peak endogenous thrombin potential (peakETP) and peak values determined by our near patient device. Importantly, patients with a peakETP below the median, experienced significantly more blood loss compared to those with a peakETP above the median. A similar division based on the peak as well as the body mass index of the patient yielded similar significant differences. A combination of the peakETP, the body mass index, and the lag-time even resulted in a better predictor of blood loss compared to each parameter separately.

Conclusions Our adapted whole blood TG assay can be used near patients and is indicative for the amount of blood loss post cardiothoracic surgery. 


\section{Introduction}

The global haemostatic assay that measures thrombin generation (TG) in plasma increasingly gained attention as a diagnostic tool in the field of thrombosis and haemostasis. TG can be used to distinguish mild from severe bleeding phenotypes in haemophilia A patients with comparable factor VIII levels ${ }^{1}$. Conventional assays, such as the prothrombin time and activated partial thromboplastin time, are based on measuring clotting times. In vitro fibrin formation, however, occurs when less than $5 \%$ of the total amount of thrombin has been formed ${ }^{2}$. This indicates that the whole haemostatic capacity cannot be adequately evaluated by an endpoint clotting assay ${ }^{3,4}$.

The calibrated automated thrombography (CAT) ${ }^{4}$ uses platelet-poor (PPP) or platelet-rich (PRP) plasma and a fluorogenic thrombin-sensitive substrate to determine the rate and extent of TG after initiation of coagulation. Coagulation is triggered with tissue factor (TF) (extrinsic pathway) or an intrinsic pathway initiator (e.g. kaolin or ellagic acid). Recently, the CAT-method was modified enabling the measurement of TG in whole blood by using a thin layer methodology, ${ }^{5,6}$. By applying TG in whole blood, the assay includes the contribution of blood cells to coagulation. Moreover, avoiding the preparation of PRP or PPP our whole blood approach reduces the assay time and thereby the time it takes to get the patient's result. Another advantage is that the method uses a low volume of blood per experiment ${ }^{6}$. Interestingly, these properties of the novel whole blood method allow the development of a near patient TG test.

The key objective of near patient testing is to have a device that is able to generate results in an acceptable time, without the need of highly skilled laboratory staff ${ }^{7}$. As a result, appropriate treatment could be determined in a shorter time-period improving clinical outcome. Other global tests to study haemostasis (e.g. viscoelastic tests) are increasingly used in the diagnosis and treatment of coagulation abnormalities in critically ill patients ${ }^{8,9}$. In addition, the overall cost of such methods is considerably lower. Current haemostatic techniques for near patient clinical use include whole blood platelet function tests (aggregometry) ${ }^{10}$, viscoelastic tests of fibrin formation (thromboelastometry) ${ }^{11}$, activated clotting time and International Normalized Ratio measurements. However, each of these assays is limited in its ability to fully evaluate a patient's coagulation profile and hence prediction of bleeding risk or thrombotic complications.

Near patient TG testing has been previously hinted ${ }^{12-15}$. First approaches were based on either an electrochemical principle ${ }^{14}$ or fluorescence principle ${ }^{15}$. To the best of our knowledge, none of these methods have been technically and clinically validated. In this paper, we report the development of a miniaturized whole blood 
TG test and a quick thrombogram analysis. The clinical potential and practicality of the novel TG assay was tested in a population of patients $(n=33)$ undergoing elective coronary artery bypass grafting (CABG), a vulnerable patient group in which post-surgical bleeding is a frequently occurring complication.

\section{Materials and methods}

\section{Healthy controls and patients}

After approval of the local medical ethical board (Medical Ethical Committee of Maastricht University Medical Centre) 5 healthy adult volunteers not taking any drugs for at least two weeks gave full informed consent according to the Helsinki declaration. Blood was collected aseptically by antecubital puncture into vacuum tubes (1 volume trisodium citrate $0.105 \mathrm{M}$ to 9 volumes blood) (BD Vacutainer System). The blood was kept at room temperature $\left(21^{\circ} \mathrm{C}\right)$ and used within 4 hours after withdrawal. Platelet-poor plasma (PPP) was obtained by double centrifugation at $2630 \mathrm{~g}$ for $10 \mathrm{~min}$ and stored at $-80^{\circ} \mathrm{C}$ until further analysis.

The cardiothoracic surgery study was approved by the local medical ethical board (Medical Ethical Committee of Maastricht University Medical Centre) and written informed consent was obtained from all volunteers. In total, 47 patients undergoing surgery for elective CABG were included. Exclusion criteria were age < 18 years and duration of extra-corporeal circulation $(E C C)<60$ min. 14 out of the 47 patients were excluded, 12 of which had a duration of the ECC of $<60 \mathrm{~min}$ and 2 underwent additional surgical procedures besides the CABG. Blood samples, taken at pre-bypass before heparin administration, were withdrawn from the arterial line after discarding the first $10 \mathrm{ml}$ and collected into vacuum tubes (1 volume trisodium citrate $0.105 \mathrm{M}$ to 9 volumes blood) (BD Vacutainer System).

\section{Clinical management}

After being connected to standard monitoring (electrocardiography, non-invasive blood pressure, pulsoximetry), all patients received a venous (Vasofix Safety, 16G, $1.7 \times 50 \mathrm{~mm}$ ) and arterial line (Radial Artery Catheterization set, 20G, $1.1 \times 44.5 \mathrm{~mm}$ Arrow International) with $2 \mathrm{ml}$ xylocaine $1 \%$ as local anaesthesia. Anaesthesia was introduced according to local standards (etomidate, sufentanil and rocuronium in a weight adapted dose) and patients were orally intubated. A large bore central venous line was placed in the right jugular vein and upon clinical need a TEE-probe was inserted for perioperative echocardiography. Anaesthesia was maintained by infusion of propofol $3-4 \mathrm{mg} / \mathrm{kg} / \mathrm{h}$ and repeated injections of sufentanil boli according to the clinical need. Before initiation of cardiopulmonary bypass (CPB) anticoagulation was achieved by administration of 300-400 IU/Kg unfractionated heparin (Heparin Leo, Leo Pharmaceutical Products BV) to achieve an activated clotting time above $400 \mathrm{~s}$. After termination of the CPB the initial heparin dose was 
reversed with protamine in a 1:1 ratio. Components of the CPB system were poly2-methoxyethylacrylate coated (Terumo). The primer for the CPB circuit consisted of $1300 \mathrm{ml}$ of $4 \%$ gelofusin, $200 \mathrm{ml} 20 \%$ mannitol, $100 \mathrm{ml} 20 \%$ human albumin, 50 $\mathrm{ml} 8.4 \% \mathrm{NaHCO}_{3}$ and $6500 \mathrm{IU}$ unfractionated heparin. Patients were kept at normothermia $\left(36^{\circ} \mathrm{C}\right)$ during CPB. Pericardial, pleural and residual blood of the CPB circuit was drained and washed with a cell saver device at the end of the perfusion. The transfusion trigger was set at a haematocrit below $23 \%$.

Pre-operative data collection included demographics and drug usage. Perioperatively, medication, infusion volumes, transfusion requirements, time on bypass, cross-clamping time, duration of the surgery, number of grafts, necessity of re-thoracotomy and blood loss were recorded. Post-operatively, the infusion volumes, transfusion requirements and the blood loss were recorded. The latter was assessed by the drainage amount of the chest tubes, which are inserted prior to closing the chest until 24 hours after surgery, when they are removed and the patient is discharged to the medium care (further referred to as "drainage volume").

\section{Haematocrit, platelet counts and fibrinogen concentration} measurements

Haematocrit and platelet counts were determined using a coulter counter (Coulter LH750 haematology analyser, Beckman Coulter Inc.). Fibrinogen concentrations were determined using a coagulation analyser according to the manufacturer's instructions (STA-R, Diagnostica Stago).

\section{Miniaturized TG device and microfluidic chip}

The measurement module of the device is a dual canal fluorescence detector (ESELog, Qiagen Lake Constance, Germany). Due to its low operating voltage and lack of moving parts, the detector can easily be integrated into various measurement processes. The detector works with impinging light and is equipped with confocal optics. It is connected via a mini to micro-USB cable to an android smartphone equipped with dedicated software (Synapse BV, Maastricht, the Netherlands) for data collection (figure 1A). The detector, holder for microfluidic chip, and temperature control system are placed in a custom housing with dimensions $13 \times 9 \times 6 \mathrm{~cm}$ (figure $1 \mathrm{~A})$. Temperature $\left(37^{\circ} \mathrm{C}\right)$ control of the chip and its holder is performed with a foil heater, temperature sensor and controller (Minco, by Alflex the Netherlands). The TG reaction in whole blood is carried out in a microfluidic chip (figure 1B) moulded from methyl methacrylate-acrylonitrilebutadiene-styrene (MABS) (chips are injection moulded by Mekora, Aachen Germany). The chip consists of two separately moulded pieces of MABS with a porous matrix disc of $5 \mathrm{~mm}$ in diameter and $160 \mu \mathrm{m}$ thickness (589/1; Whatman, Germany) in their centre. Before assembly the part that bears the microfluidic 
channels is treated with a plasma-cleaner (PDC-32G-2, Harrick Plasma, NY, USA) for 15 minutes.
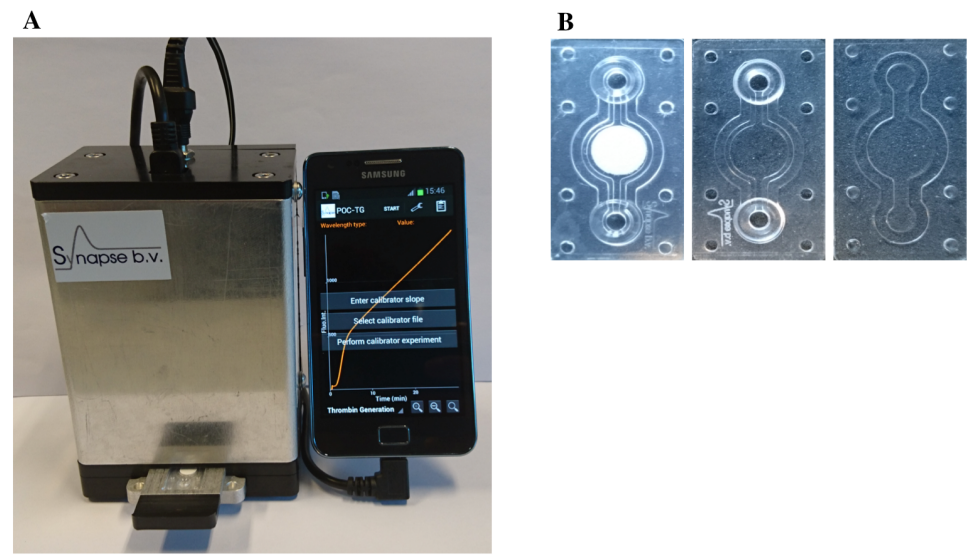

Figure 1. Presentation of the near patient TG system.

(A) The image of the prototype device and connected smartphone with dedicated software. (B) Image of an assembled (left) and disassembled (right) two-layer transparent microfluidic (MABS) chip with a porous matrix disc of $5 \mathrm{~mm}$ in diameter and $160 \mu \mathrm{m}$ thickness.

\section{Near Patient TG Assay}

Citrated blood $(15 \mu \mathrm{L})$ was mixed with $15 \mu \mathrm{L}$ activator solution containing $(\mathrm{ZGGR})_{2^{-}}$ Rhodamine 110 ( $\mathrm{P}_{2} \mathrm{Rho}$ ), recombinant human tissue factor (TF; Innovin ${ }^{\circledR}$, Dade Behring, Germany) and $\mathrm{CaCl}_{2}$ in $20 \mathrm{mM} \mathrm{HEPES}, 140 \mathrm{mM} \mathrm{NaCl}$ and $5 \mathrm{mg} / \mathrm{mL}$ bovine serum albumin (Sigma, the Netherlands) with a pH of 7.35. The $P_{2}$ Rho was a kind gift of Diagnostica Stago, (France). The final concentrations are $50 \%$ volume blood, $1 \mathrm{pM} \mathrm{TF}, 16.7 \mathrm{mM} \mathrm{CaCl}_{2}$, and $300 \mu \mathrm{M} \mathrm{P}{ }_{2}$ Rho. To calibrate the fluorescence signal, a second measurement was performed in which $5 \mu \mathrm{L} \mathrm{P}_{2}$ Rho and $10 \mu \mathrm{L}$ $\alpha 2$ macroglobulin-thrombin ( $\alpha 2 \mathrm{M}-\mathrm{T}$ ) complex (prepared as previously described ${ }^{16}$ ) were added to the blood. The final thrombin activity at $100 \mathrm{nM}$ was used for calibration of the experiments. The fluorescence signal was recorded with $\gamma_{\mathrm{ex}}=485$ $\mathrm{nm}$ and $\gamma_{\mathrm{em}}=538 \mathrm{~nm}$. The assay was performed at $37^{\circ} \mathrm{C}$.

\section{Models for Thrombogram Analysis}

The traditional CAT algorithm describes the thrombin generating capacity of a person from a thrombogram, using the parameters thrombin peak height (peak, $n M)$, area under the curve (ETP, endogenous thrombin potential, nM.min), time to reach $5 \mathrm{nM}$ of thrombin (lag-time, $\mathrm{min}$ ), and time-to-peak (ttpeak, min) ${ }^{4}$. The timeconsuming nature makes the CAT method unsuitable for fast near patient testing. We assumed that the sigmoidal part of the fluorescence curve, until its inflection point, provides relevant information regarding the thrombin generating capacity of 
a person. The sigmoidal part of the curve was fitted with a flexible, extended Chapman-Richards growth (CRG) equation, $F=a\left(1-e^{-b t}\right) c+d\left(1-e^{-f t}\right)^{17}$, where $F$ is the fluorescence intensity at time $t$ and $a, b, c, d, f$, and $g$ are the parameters that determine the shape of the curve. Subsequently, the peakETP (area under the curve until the peak is reached), peak, lag-time, and time-to-peak (ttpeak) were calculated from the first derivative of the simulated curve.

\section{PPP CAT}

PPP CAT was performed as previously described ${ }^{4}$. Briefly, the CAT assay was measured in a pre-heated plate fluorometer (Ascent reader, Thermolabsystems OY, Helsinki, Finland). To each well, $80 \mu$ l of plasma was added in combination with the TF trigger (final concentration $1 \mathrm{pM}$ ) and phospholipid vesicles (20 mol\% phosphatidylserine, 60 mol\% phosphatidylcholine and 20 mol\% phosphatidylethanolamine from Avanti) at a final concentration of $4 \mu \mathrm{M}$ in Hepes-buffered saline.

\section{Statistics}

Statistical analysis was performed with SPSS version 21 (IBM, Inc). Results are expressed as mean and standard deviation (SD). Data were checked for normality and the Spearman or Pearson correlation was calculated accordingly, to verify the association between different parameters. In addition, patients were divided into two groups based on the median of each of the TG parameters, clinical parameters or median drainage volume. Resulting groups were compared for drainage volume and other laboratory/clinical parameters using the Mann-Whitney $U$ test for independent samples for scale variables and the Fisher's exact test for nominal variables.

\section{Results}

\section{Comparison of calculation methods}

To validate the CRG model-based data analysis procedure, we compared the thrombogram parameters calculated with the CRG model to those obtained with the CAT-based calculation method ${ }^{18,19}$. Citrated blood from one subject was activated with TF $(5 \mathrm{pM})$ and $\mathrm{CaCl}_{2}(16.7 \mathrm{mM})$ in the presence of $300 \mu \mathrm{M} \mathrm{P}{ }_{2} \mathrm{Rho}$. The increase in fluorescence intensity was recorded over time using the CAT. The CAT-based analysis is displayed in figure 2A. The calibrator data represent the cleavage of substrate by a known amount of $\alpha 2 \mathrm{M}-\mathrm{T}$ (solid line). The fluorescence intensity from the experimental mixture (dotted line) was converted in a thrombin concentration using the calibrator. In figure $2 \mathrm{~B}$ the first derivative of the calibrated curve shows the generation of free thrombin in molar concentration (solid line), after correction for the formation of a2M-T complex (dotted line), that still cleaves 
the substrate but does not represent free thrombin activity. This method covers all coagulation stages, including initiation, propagation and termination of TG.

Secondly, the same set of raw data, but only until the inflection point of the sigmoidal curve was fitted using the Chapman-Richard function ${ }^{17}$. In figure $2 \mathrm{C}$, the calibrator data (solid line), the raw data points (dotted line), and the results of the fit (solid red line) are displayed. In figure 2D the goodness of the fit is determined by the difference between the experimental and fitted data. The sum of these differences constitutes the total error of the fit. In figure $2 \mathrm{E}$ the first derivative of

A

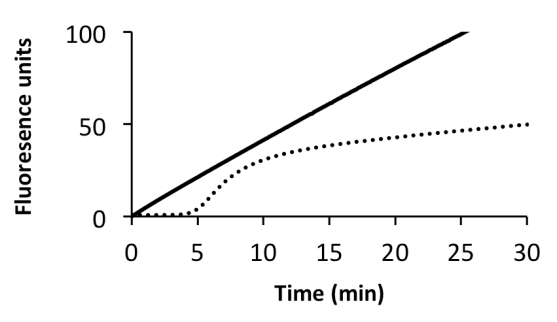

B

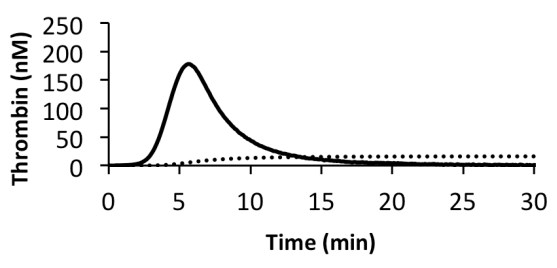

C

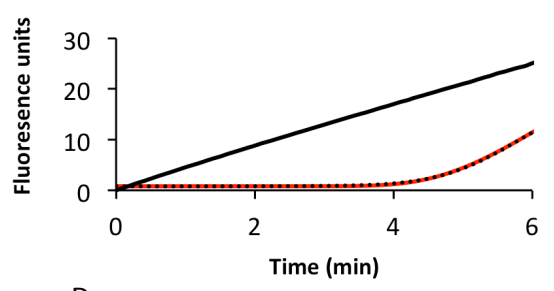

D

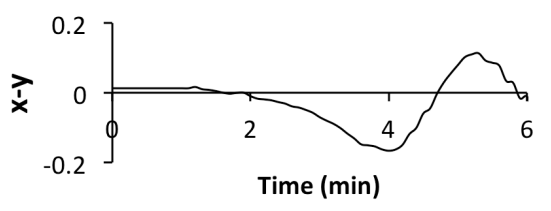

$E$

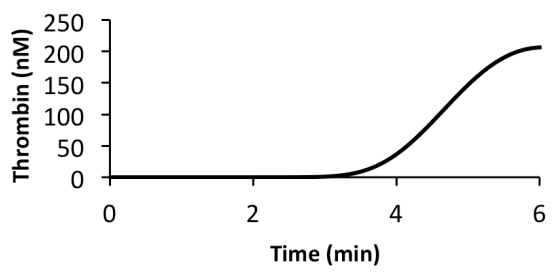

Figure 2. TG curves determined using the CAT method $(A, B)$ and the CRG method (C-E). Citrated whole blood from a healthy donor was triggered with $5 \mathrm{pM} \mathrm{TF}, 16.7 \mathrm{mM} \mathrm{CaCl} 2$ in the presence of $300 \mu \mathrm{M} \mathrm{P} \mathrm{P}_{2}$ Rho. The fluorescence intensity was recorded in triplicate measurements. (A) The raw calibrator data (solid line) and raw TG data (dashed line) are shown. (B) The first derivative of the corrected and calibrated curve (solid line) after correction for the $\alpha 2 \mathrm{M}-\mathrm{T}$ complex formation (dashed line) is shown. (C) The raw calibrator data (solid line), the raw experimental TG data (dashed line) are shown up to the inflection point, together with the fitted data using the CRG model (red solid line). (D) The residues, 
$\left(F_{\exp }-F_{\text {fit }}\right)^{2}$ resemble the goodness of the fit. (E) The first derivative of the CRG simulated curve until the inflection point is shown.

Thrombogram parameters were calculated for both the CAT and CRG method, and this approach was repeated for 4 additional healthy individuals. To compare the thrombogram parameters obtained with both models, correlation coefficients between each of the TG parameters of the two calculation methods were calculated and were $0.868,0.998,0.993$ and 0.981 for the (peak)ETP, peak, lagtime and ttpeak, respectively.

The CRG model only considers the data until the peak is reached. Therefore, the peakETP calculated with the CRG model provides limited information about the termination phase of TG, resulting in a relatively lesser correlation with the ETP parameter of the CAT model.

\section{Inter-assay precision}

The assay was tested for its accuracy in measuring the calibration factor for which the rate of increase of fluorescence intensity (FU/min) can be converted in molar amounts of thrombin. Citrated blood from one subject was repeatedly $(n=10)$ mixed with $\alpha 2 \mathrm{M}-\mathrm{T}(100 \mathrm{nM})$ in the presence of $300 \mu \mathrm{M} \mathrm{P}_{2}$ Rho. Under the experimental conditions used, we found that during the assay time (10 $\mathrm{min})$, the fluorescence intensity increased linear in time during the full course of the experiment. The coefficient of variation between the 10 calibrator measurements proved to be $13.8 \%$.

Similarly, re-calcified citrated blood from the same healthy subject was repeatedly $(n=10)$ assayed for its TF-induced (1 pM TF) thrombin generating capacity. The resulting thrombogram parameters, displayed as mean \pm SD (CV) are shown in table 1 . The inter-assay variation of the 5 healthy individuals that were measured to determine the inter-individual variation, as well as that of the 33 patients included for the clinical evaluation, was $<11 \%$ for all of the TG parameters. These results are comparable with previously published inter-assay precision in the whole blood CAT ${ }^{6}$. Intra-assay precision could not be assessed, as we are restricted to measuring one sample at a time. Moreover, inter-assay precision was not determined over a time period longer than 1 day due to the storage limitations of whole blood.

\section{Inter-individual variation}

The thrombin generating capacity of TF-activated re-calcified citrated blood from 5 healthy subjects was measured in triplicate. The mean and SD for the different parameters between the 5 subjects are displayed in table 1 . The inter-individual 
Near Patient Thrombin Generation in Patients Undergoing Elective Cardiac Surgery

variation of the TG parameters obtained with our new device are comparable to those of the whole blood CAT ${ }^{6}$. 
Table 1. Inter-individual and inter-assay variation in TG parameters.

For the inter-individual variation 5 healthy subjects were tested using the near patient TG device; for the inter-assay variation 1 healthy subject was tested 10 times. TG parameters were determined using the CRG model

\begin{tabular}{lllll}
\hline & \multicolumn{2}{l}{$\begin{array}{l}\text { Inter-individual } \\
\text { variation N }=5\end{array}$} & $\begin{array}{l}\text { Inter-assay } \\
=10\end{array}$ & variation N \\
\hline Parameter & Mean \pm SD & $\%$ CV & Mean \pm SD & $\%$ CV \\
\hline peakETP (nM.min) & $154.7 \pm 34.3$ & 22 & $133.5 \pm 19$ & 14 \\
Peak (nM) & $116.5 \pm 26.2$ & 22 & $102.5 \pm 11.9$ & 12 \\
Lag-time (min) & $3.18 \pm 0.4$ & 13 & $2.6 \pm 0.2$ & 8 \\
ttpeak (min) & $5.8 \pm 0.4$ & 7 & $5.1 \pm 0.4$ & 8 \\
\hline
\end{tabular}

\section{Near patient whole blood TG in patients undergoing cardiothoracic surgery}

To test the applicability of our new method, we studied 33 patients scheduled for CABG with the use of a heart lung machine for extracorporeal circulation. To compare the results obtained with our new method to the golden standard in TG, measurements were also performed in PPP. Patients were divided in two groups based on the median of drainage volume, resulting in a group of 'severe' and 'mild' bleeders. General patient characteristics, as well as pre- and post-operative variables of all groups are shown in table 2 in the supplemental information. A statistically significant difference was found between the two groups for the peakETP and the peak of the near patient data and for the ETP of the PPP data. The resulting parameters displayed as mean \pm SD per group with their respective $P$ value are for the peakETP $162.8 \pm 38.1$ versus $120.3 \pm 35.9(P=0.002)$, the peak $136.7 \pm 40.4$ versus $106.0 \pm 29.6(P=0.011)$ and the ETP (PPP) $1244.5 \pm 274.1$ versus $1025.1 \pm 216.4(P=0.017)$ for the severe versus mild bleeders, respectively. Other parameters that differed significantly between the two groups include the patient's weight $(87.9 \pm 10.9$ versus $76.1 \pm 13.9 ; \mathrm{P}=0.015)$, their BMI $(29.1 \pm 2.3$ versus $25.1 \pm 3.9 ; \mathrm{P}=0.001$ ), and the administered volume of colloids during the first $24 \mathrm{~h}$ on the intensive care unit $(21.4 \pm 10.8$ versus $9.2 \pm 6.4 ; \mathrm{P}<0.001)$.

Alternatively, to determine the predictive potential of different parameters for bleeding, patients were divided in two groups based on the median of the general characteristics, the peri- and post-operative variables, and the clinical characteristics. Next, the difference in drainage volume, was compared between these two groups (table 3 in the supplemental information). A statistically significant difference in drainage volume was found when patients were divided based on the median BMI, post-operative colloid administration, the peakETP, the 
peak parameters of the near patient data, and the pre-operative fibrinogen concentration. Individual data of the drainage volume for the two groups stratified according to the median of the peakETP and BMI are shown in figure $3 \mathrm{~A}$, and figure $3 B$, respectively. Interestingly, the combination of peakETP, lag-time and $\mathrm{BMI}$ further enhanced the prediction of the drainage volume (figure $3 \mathrm{C}$ ).

A

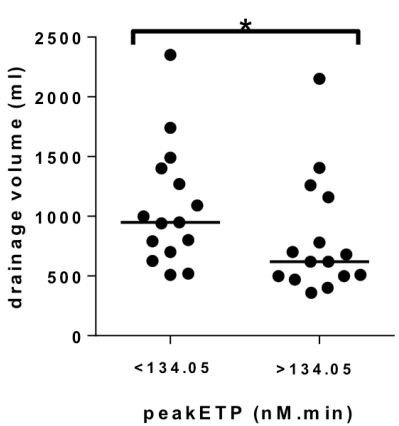

B

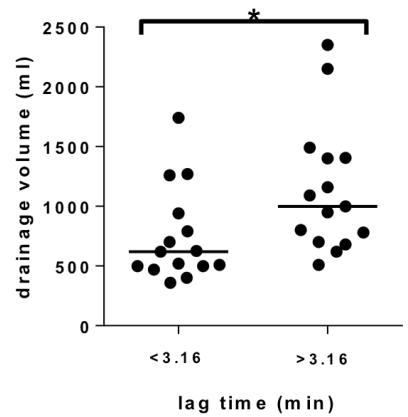

C

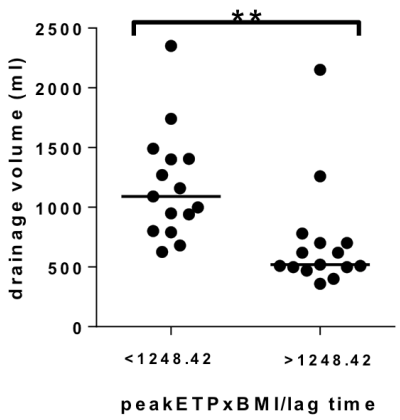

Figure 3. Drainage volume in patients undergoing cardiothoracic surgery stratified according the median peakETP (A) as assessed by our near patient device, the median BMI (B), and a combined parameter based on the peakETP, lag-time and BMI (C).

33 patients undergoing cardiothoracic surgery were divided into two groups based on either the median peak-ETP (A), BMI (B), or a combined parameter based on the peakETP, lag-time and BMI. Individual values of drainage volume are shown, together with the mean \pm SEM. ${ }^{*} p<0.05,{ }^{* *} p<0.005$, and ${ }^{* * *} p<0.001$ (Mann-Whitney $U$ test)

\section{Discussion}

The present study demonstrates the technical development and clinical testing of a whole blood TG method as a near patient test. Our new whole blood device is characterized by a good technical performance, comparable to the standard CAT method. The high turnover time (up to $60 \mathrm{~min}$ ) is still a major bottleneck for the current $\mathrm{TG}^{20}$. Not only have we been able to reduce the size of the device, with our CRG calculation model we decreased the turnover time of our new test to less than $20 \mathrm{~min}$. To achieve the short measurement time, an altered calculation method is needed, only considering the data until the peak of the TG curve.

We tested the clinical applicability of the device in a population of patients $N=33$ undergoing elective CABG. These patients are known to have a high bleeding risk due to the invasive nature of the surgery ${ }^{21,22}$. The extracorporeal circulation is believed to be one of the main contributors to the bleeding risk. The circuit causes major haemostatic disturbances e.g. due the dilution of coagulation factors as a result of the priming fluid of the machine ${ }^{23,24}$. Currently there are no tests available 
that accurately predict the risk of blood loss of a patient planned to undergo surgery. The coagulation tests that are performed pre-operatively are mainly used to identify coagulation disorders that increase the risk of peri-operative bleeding ${ }^{25}$. However, these tests lack the sensitivity to pick up subtle inter-individual differences between patients that do not have any obvious coagulopathy. The more recently emerged viscoelastic tests for near patient testing, including thromboelastography and rotational thromboelastometry, are primarily used to aid in the selection of the optimal allogeneic blood products or coagulation factor concentrates during/after clinical bleeding ${ }^{20}$. However, these viscoelastic tests have little predictive value in terms of peri-operative blood loss ${ }^{26,27}$.

TG measurements in plasma proved to be indicative for blood loss in patients undergoing $\mathrm{CABG}^{28,29}$. Despite the relatively small number of patients included, our study confirms these results, as patients with a volume below the median drainage volume, were characterized by a significantly lower ETP in plasma. Dividing patients based on the median of the drainage volume, also resulted in significant differences in peakETP and peak determined by the near patient whole blood TG device, as well as the BMI. This is in accordance with other studies that have shown that patients with a lower BMI are at a higher risk regardless of the type of cardiac surgery with $\mathrm{CPB}^{30,31}$.

To determine the predictive capacity of our near patient measurements, we divided patients based on the median of pre- and postoperative determined parameters, and compared the drainage volume between both groups. Interestingly, patients with a peakETP below the median, experienced significantly more drainage volume compared to those with a peakETP above the median. Moreover, a division based on the peak data of our new device resulted in a similar significant difference between the two groups.

Besides our near patient TG parameters, the BMI was a predictive indicator for the amount of drainage volume when subjected to this form of analysis. Interestingly, the combination of the peakETP, the lag-time, and the BMI of the patient, proved to be a better predictor of the drainage volume than each parameter separately. In accordance with previous results, the pre-operative fibrinogen concentration shows a similar trend ${ }^{32}$.

We do acknowledge the limitation of the study size and understand that, despite the statistical significance, this study should be extended to larger patient populations. Additionally, further clinical studies are necessary to determine its importance in the monitoring and therapeutic guidance of other bleeding and thrombotic pathologies. A possible limitation of our device is that, due to the external mixing of reagents, a technician and laboratory environment are still 
necessary. We are currently busy with the implementation of the reagents in the chips. Furthermore, we are optimizing a quality control that can be run daily to verify proper functioning of the device. Before implementation of the device in clinical settings, the effect of common interferents such as bilirubin, lipid particles and commonly co-administered drugs should be further investigated. Of note, the haemoglobin and creatinine levels pre-surgery did not correlate with the presurgery TG parameters (data not shown). Indirect effects of co-administered drugs and other surgery-specific effects should be assessed prior to clinical use of our device. In this study, the patient group was too small to draw any conclusions regarding these effects. Of course, anticoagulant drugs will have an influence on the outcome of our test as they influence the coagulation system of the patient. Usually, patients are asked to discontinue this medication before surgery, but any remaining of these medications will influence TG and hence blood loss.

Importantly, despite the small sample size and the described limitations, our data suggest an added clinical value for our TG device being indicative of the bleeding risk after cardiac surgery. A test, such as the near patient TG, that can be performed in the vicinity of the patient and hence gives a fast result, has the added benefit that treatment can be initiated faster. This in turn makes the treatment more effective as time is of utmost importance in a clinical setting ${ }^{33}$. Ultimately, after further validation and simplification, our device may be suitable for use outside a laboratory/hospital environment e.g. in the general practitioner's office. This will reduce the number of routine hospital visits and therewith improve the quality of life of patients. 


\section{References}

1. Santagostino, E. et al. Severe hemophilia with mild bleeding phenotype: molecular characterization and global coagulation profile. J. Thromb. Haemost. 8, 737-743 (2010).

2. Mann, K. G., Brummel, K. \& Butenas, S. What is all that thrombin for? J. Thromb. Haemost. 1, 1504-1514 (2003).

3. Young, G. et al. Thrombin generation and whole blood viscoelastic assays in the management of hemophilia: current state of art and future perspectives. Blood 121, 19441950 (2013).

4. Hemker, P. G. H. C. The calibrated automated thrombogram (CAT): a universal routine test for hyper- and hypocoagulability. Pathophysiol. Haemost. Thromb. 32, 249-53 (2002).

5. Nijhuis, S., Apitz-Castro, R. \& Hemker, H. Thrombin generation in a thin layer of whole blood. J Thromb Haemost 7, 669 (2009).

6. Ninivaggi, M. et al. Whole-Blood Thrombin Generation Monitored with a Calibrated Automated Thrombogram-Based Assay. Clin. Chem. 58, 1252-1259 (2012).

7. Gubala, V., Harris, L. F., Ricco, A. J., Tan, M. X. \& Williams, D. E. Point of Care Diagnostics: Status and Future. Anal. Chem. 84, 487-515 (2012).

8. Goodnough, L. T. \& Hill, C. C. Use of point-of-care testing for plasma therapy. Transfusion (Paris) 52, 56S-64S (2012).

9. Meybohm, P., Zacharowski, K. \& Weber, C. F. Point-of-care coagulation management in intensive care medicine. Crit. Care 17, 218 (2013).

10. Harle, C. C. Point-of-Care Platelet Function Testing. Semin. Cardiothorac. Vasc. Anesth. 11, 247-251 (2007).

11. Hobson, A. R., Agarwala, R. A., Swallow, R. A., Dawkins, K. D. \& Curzen, N. P. Thrombelastography: Current clinical applications and its potential role in interventional cardiology. Platelets 17, 509-518 (2006).

12. Kost, G. J. et al. Point-of-Care Testing for Disasters: Needs Assessment, Strategic Planning, and Future Design. Clin. Lab. Med. 29, 583-605 (2009).

13. Ten Cate, H. Thrombin generation in clinical conditions. Thromb. Res. 129, 367-370 (2012).

14. Thuerlemann, C., Haeberli, A. \& Alberio, L. Monitoring Thrombin Generation by Electrochemistry: Development of an Amperometric Biosensor Screening Test for Plasma and Whole Blood. Clin. Chem. 55, 505-512 (2009).

15. Koch, K. et al. Thrombin generation test in microfluidic systems. J. Appl. Phys. 105, 102012 (2009).

16. Hemker, H. C. et al. Calibrated Automated Thrombin Generation Measurement in Clotting Plasma. Pathophysiol. Haemost. Thromb. 33, 4-15 (2003).

17. Richards, F. J. A Flexible Growth Function for Empirical Use. J. Exp. Bot. 10, 290-301 (1959).

18. Hemker, H. C. \& Kremers, R. Data management in Thrombin Generation. Thromb. Res. 131, 3-11 (2013).

19. Wagenvoord, R., Hemker, P. W. \& Hemker, H. C. The limits of simulation of the clotting system. J. Thromb. Haemost. 4, 1331-1338 (2006).

20. Bolliger, D. \& Tanaka, K. A. Roles of Thrombelastography and Thromboelastometry for Patient Blood Management in Cardiac Surgery. Transfus. Med. Rev. 27, 213-220 (2013).

21. Woodman, R. C. \& Harker, L. A. Bleeding complications associated with cardiopulmonary bypass. Blood 76, 1680-1697 (1990).

22. Christensen, M. C., Krapf, S., Kempel, A. \& von Heymann, C. Costs of excessive postoperative hemorrhage in cardiac surgery. J. Thorac. Cardiovasc. Surg. 138, 687-693 (2009).

23. Schols, S. E. M. et al. Impaired thrombin generation and fibrin clot formation in patients with dilutional coagulopathy during major surgery. Thromb. Haemost. 103, 318-328 (2010). 
24. Davidson, S. State of the Art - How I manage coagulopathy in cardiac surgery patients. Br. J. Haematol. 164, 779-789 (2014).

25. Fowler, A. \& Perry, D. J. Laboratory monitoring of haemostasis. Anaesthesia 70, 68-72 (2015).

26. Tanaka, K. A., Bolliger, D., Vadlamudi, R. \& Nimmo, A. Rotational Thromboelastometry (ROTEM)-Based Coagulation Management in Cardiac Surgery and Major Trauma. J. Cardiothorac. Vasc. Anesth. 26, 1083-1093 (2012).

27. Spahn, D. R. \& Goodnough, L. T. Alternatives to blood transfusion. The Lancet 381, 18551865 (2013).

28. Bosch, Y. et al. Preoperative thrombin generation is predictive for the risk of blood loss after cardiac surgery: a research article. J. Cardiothorac. Surg. 8, 154 (2013).

29. Bosch, Y. P. J. et al. Measurement of thrombin generation intra-operatively and its association with bleeding tendency after cardiac surgery. Thromb. Res. 133, 488-494 (2014).

30. Firanescu, C. E., Martens, E. J., Schönberger, J. P. A. M., Hamad, M. A. S. \& Straten, A. H. M. van. Postoperative blood loss in patients undergoing coronary artery bypass surgery after preoperative treatment with clopidogrel. A prospective randomised controlled study. Eur. J. Cardiothorac. Surg. 36, 856-862 (2009).

31. Carroll, R. C., Chavez, J. J., Snider, C. C., Meyer, D. S. \& Muenchen, R. A. Correlation of perioperative platelet function and coagulation tests with bleeding after cardiopulmonary bypass surgery. J. Lab. Clin. Med. 147, 197-204 (2006).

32. Karlsson, M. et al. Plasma fibrinogen level, bleeding, and transfusion after on-pump coronary artery bypass grafting surgery: a prospective observational study. Transfusion (Paris) 48, 2152-2158 (2008).

33. Görlinger, K. et al. First-line Therapy with Coagulation Factor Concentrates Combined with Point-of-Care Coagulation Testing is Associated with Decreased Allogeneic Blood Transfusion in Cardiovascular Surgery A Retrospective, Single-center Cohort Study. J. Am. Soc. Anesthesiol. 115, 1179-1191 (2011). 


\section{Supplementary information}

Table 2. Distribution of patient characteristics, peri- and post-operative variables. Patients are divided into two groups based on the median of the drainage volume. Group 1 had a drainage volume of $<780 \mathrm{ml}$ group 2 had a drainage volume $>780 \mathrm{ml}$.

\begin{tabular}{|c|c|c|c|c|c|c|c|}
\hline \multirow[t]{3}{*}{ Variable } & \multicolumn{2}{|c|}{ All patients } & \multicolumn{2}{|c|}{ Group 1} & \multicolumn{2}{|c|}{ Group 2} & \multirow{3}{*}{$\begin{array}{l}\text { P-value } \\
\text { Mann- } \\
\text { Whitney U- } \\
\text { test / } \\
\text { Fisher's } \\
\text { exact test }\end{array}$} \\
\hline & \multicolumn{2}{|l|}{$N=33$} & \multicolumn{2}{|l|}{$N=17$} & \multicolumn{2}{|l|}{$N=16$} & \\
\hline & Mean & SD & Mean & SD & Mean & SD & \\
\hline \multicolumn{8}{|l|}{ General characteristics } \\
\hline Age (years) & 66.8 & 8.7 & 67.1 & 10.0 & 66.5 & 7.5 & 0.958 \\
\hline Gender (\% female) & $24 \%$ & & $35 \%$ & & $13 \%$ & & 0.225 \\
\hline Length (cm) & 173.6 & 7.8 & 173.5 & 7.6 & 173.8 & 8.5 & 1.000 \\
\hline Weight (kg) & 82.2 & 13.6 & 87.9 & 10.9 & 76.1 & 13.9 & 0.015 \\
\hline BMI & 27.2 & 3.7 & 29.1 & 2.3 & 25.1 & 3.9 & 0.001 \\
\hline \multicolumn{8}{|l|}{ Intraoperatively } \\
\hline Heparin-total (IU/kg) & 94.6 & 59.1 & 99.2 & 75.3 & 89.8 & 36.8 & 0.510 \\
\hline Protamin-total (mg/kg) & 3.6 & 0.5 & 3.4 & 0.4 & 3.8 & 0.6 & 0.087 \\
\hline Tranexamic acid (mg/kg) & 36.8 & 13.2 & 32.7 & 6.0 & 41.2 & 17.1 & 0.063 \\
\hline Crystalloids (ml/kg) & 14.7 & 9.8 & 11.6 & 7.2 & 17.3 & 11.5 & 0.168 \\
\hline Colloids (ml/kg) & 0.7 & 1.7 & 0.7 & 1.5 & 0.7 & 1.9 & 1.000 \\
\hline Total packed cells (ml/kg) & 0.6 & 2.1 & 0.2 & 0.9 & 1.1 & 2.9 & 0.736 \\
\hline $\mathrm{FFP}(\mathrm{ml} / \mathrm{kg})$ & 0.0 & 0.0 & 0.0 & 0.0 & 0.0 & 0.0 & NA \\
\hline Platelet concentrates (ml/kg) & 0.3 & 1.3 & 0.0 & 0.0 & 0.7 & 1.9 & 0.557 \\
\hline Fibrinogen $(\mathrm{mg} / \mathrm{kg})$ & 1.3 & 5.4 & 0.0 & 0.0 & 2.6 & 7.7 & 0.557 \\
\hline Fluid in Cell Saver (ml) & 439.2 & 230.2 & 474.9 & 240.0 & 401.3 & 220.8 & 0.345 \\
\hline Fluid in Ultrafiltration (ml) & 333.3 & 578.1 & 317.7 & 604.4 & 350.0 & 568.0 & 0.873 \\
\hline Time on bypass (min) & 82.0 & 17.3 & 81.1 & 17.6 & 83.1 & 17.5 & 0.557 \\
\hline Time X-clamping (min) & 59.3 & 16.1 & 59.2 & 17.1 & 59.4 & 15.6 & 0.790 \\
\hline Duration surgery (min) & 205.0 & 45.0 & 205.5 & 49.8 & 204.6 & 40.9 & 0.763 \\
\hline Number of CABG & 3.6 & 1.1 & 3.4 & 1.3 & 3.9 & 0.8 & 0.465 \\
\hline \multicolumn{8}{|l|}{ Postoperatively } \\
\hline Crystalloids (ml/kg) & 27.8 & 10.0 & 26.5 & 9.1 & 29.3 & 11.0 & 0.276 \\
\hline Colloids (ml/kg) & 15.1 & 10.7 & 9.2 & 6.4 & 21.4 & 10.8 & 0.000 \\
\hline Total packed cells (ml/kg) & 3.6 & 7.3 & 0.4 & 1.1 & 6.9 & 9.5 & 0.053 \\
\hline $\mathrm{FFP}(\mathrm{ml} / \mathrm{kg})$ & 1.0 & 3.5 & 0.0 & 0.0 & 2.0 & 4.9 & 0.363 \\
\hline Platelet concentrates (ml/kg) & 1.0 & 2.2 & 0.0 & 0.0 & 2.0 & 2.8 & 0.068 \\
\hline Fibrinogen (mg/kg) & 3.2 & 10.2 & 0.0 & 0.0 & 6.5 & 14.1 & 0.363 \\
\hline Re-thoracotomy (\% of patients) & $9 \%$ & & $0 \%$ & & $18.8 \%$ & & 0.103 \\
\hline $\begin{array}{l}\text { Laboratory parameters befo } \\
\text { surgery }\end{array}$ & & & & & & & \\
\hline
\end{tabular}




\begin{tabular}{|c|c|c|c|c|c|c|c|}
\hline peakETP (nM.min) & 142.2 & 42.4 & 162.8 & 38.1 & 120.3 & 35.9 & 0.002 \\
\hline peak (nM) & 121.8 & 38.4 & 136.7 & 40.4 & 106.0 & 29.6 & 0.011 \\
\hline lag-time (min) & 3.2 & 0.5 & 3.2 & 0.4 & 3.3 & 0.5 & 0.309 \\
\hline ttpeak (min) & 5.3 & 0.7 & 5.3 & 0.7 & 5.3 & 0.7 & 0.958 \\
\hline PPP ETP (nM.min) & $\begin{array}{l}1138 . \\
1\end{array}$ & 268.2 & 1244.5 & 274.1 & 1025.1 & 216.4 & 0.017 \\
\hline PPP peak (nM) & 267.4 & 75.6 & 286.6 & 78.5 & 246.9 & 69.0 & 0.094 \\
\hline PPP lag-time (min) & 4.2 & 1.9 & 4.4 & 2.0 & 4.0 & 1.9 & 0.231 \\
\hline PPP ttpeak (min) & 6.4 & 2.3 & 6.6 & 2.5 & 6.2 & 2.2 & 0.363 \\
\hline Fibrinogen concentration (g/L) & 3.6 & 0.8 & 3.9 & 0.9 & 3.4 & 0.6 & 0.127 \\
\hline \multicolumn{8}{|l|}{ Blood loss } \\
\hline Blood loss during surgery (ml) & $\begin{array}{l}1514 . \\
6\end{array}$ & 699.0 & 1529.4 & 586.5 & 1499.0 & 821.5 & 0.465 \\
\hline Drainage volume (ml) & 928.2 & 485.3 & 585.0 & 123.8 & 1292.8 & 459.1 & 0.000 \\
\hline Total blood loss (ml) & $\begin{array}{l}2442 . \\
8\end{array}$ & 894.2 & 2114.4 & 517.1 & 2791.5 & $\begin{array}{l}1080 . \\
9\end{array}$ & 0.031 \\
\hline
\end{tabular}

BMI, Body Mass Index; CABG, Coronary Artery Bypass Grafting; ETP, Endogenous Thrombin Potential; ttpeak, time-to-peak; FFP, Fresh Frozen Plasma; SD, standard deviation.

Table 3. Distribution of the drainage volume. Patients are divided into two groups based on the median of the indicated parameter.

\begin{tabular}{|c|c|c|c|c|c|}
\hline \multirow[t]{3}{*}{ Variable } & \multirow{2}{*}{\multicolumn{2}{|c|}{$\begin{array}{l}<\text { Median } \\
N=17\end{array}$}} & \multirow{3}{*}{\multicolumn{2}{|c|}{$\begin{array}{l}>\text { Median } \\
\mathrm{N}=16 \\
\text { Mean SD }\end{array}$}} & \multirow{3}{*}{$\begin{array}{l}\text { P-value } \\
\text { Mann-Whitney U- } \\
\text { test }\end{array}$} \\
\hline & & & & & \\
\hline & Mean & SD & & & \\
\hline \multicolumn{6}{|l|}{ General characteristics } \\
\hline Age (years) & 944.7 & 580.2 & 910.6 & 377.7 & 0.683 \\
\hline Length $(\mathrm{cm})$ & 1025.0 & 598.6 & 825.3 & 313.6 & 0.557 \\
\hline Weight (kg) & 1030.9 & 521.5 & 819.1 & 433.3 & 0.157 \\
\hline BMI & 1103.2 & 484.3 & 742.19 & 424.9 & 0.005 \\
\hline \multicolumn{6}{|l|}{ Intraoperatively } \\
\hline Heparin-total (IU/kg) & 825.6 & 407.7 & 1037.2 & 548.2 & 0.276 \\
\hline Protamin-total (mg/kg) & 943.4 & 481.3 & 913.8 & 503.4 & 0.929 \\
\hline Transexamin acid (mg/kg) & 771.5 & 275.5 & 1094.7 & 603.4 & 0.136 \\
\hline Crystalloids (ml/kg) & 933.2 & 514.0 & 922.8 & 569.7 & 0.736 \\
\hline Fluid in Cell Saver (ml) & 928.2 & 302.9 & 928.1 & 635.1 & 0.231 \\
\hline Time on bypass (min) & 814.7 & 344.9 & 1048.8 & 587.7 & 0.292 \\
\hline Duration X-clamping (min) & 898.8 & 470.9 & 959.9 & 513.7 & 0.736 \\
\hline Duration surgery (min) & 887.1 & 385.1 & 971.8 & 583.3 & 0.958 \\
\hline Number of CABG & 778.8 & 302.9 & 1086.9 & 593.6 & 0.118 \\
\hline \multicolumn{6}{|l|}{ Postoperatively } \\
\hline Crystalloids (ml/kg) & 862.4 & 464.8 & 998.1 & 511.7 & 0.382 \\
\hline Colloids (ml/kg) & 738.2 & 313.1 & 1130.0 & 559.9 & 0.019 \\
\hline
\end{tabular}




\begin{tabular}{|c|c|c|c|c|c|}
\hline $\begin{array}{l}\text { Laboratory parameters be } \\
\text { surgery }\end{array}$ & & & & & \\
\hline peakETP (nM.min) & 1086.5 & 480.3 & 760.0 & 444.7 & 0.006 \\
\hline peak (nM) & 1049.7 & 476.3 & 799.1 & 475.3 & 0.031 \\
\hline lag-time (min) & 856.2 & 536.3 & 1004.7 & 428.3 & 0.094 \\
\hline ttpeak (min) & 897.9 & 539.8 & 960.3 & 435.3 & 0.309 \\
\hline PPP ETP (nM.min) & 1070.3 & 558.3 & 777.2 & 350.3 & 0.118 \\
\hline PPP peak (nM) & 1024.1 & 573.6 & 826.3 & 360.5 & 0.382 \\
\hline PPP lag-time (min) & 932.9 & 408.9 & 923.1 & 569.2 & 0.510 \\
\hline PPP ttpeak (min) & 968.2 & 388.7 & 885.6 & 580.9 & 0.146 \\
\hline Fibrinogen concentration $(\mathrm{g} / \mathrm{L})$ & 1114.4 & 565.8 & 730.3 & 282.6 & 0.034 \\
\hline \multicolumn{6}{|l|}{ Blood loss } \\
\hline Blood loss during surgery (ml) & 914.7 & 366.7 & 912.0 & 612.0 & 0.136 \\
\hline Total blood loss (ml) & 726.2 & 236.7 & 1142.8 & 589.3 & 0.041 \\
\hline
\end{tabular}

BMI, Body Mass Index; CABG, Coronary Artery Bypass Grafting; ETP, Endogenous Thrombin Potential; ttpeak, time-to-peak. 


Chapter 3

\title{
Thrombin Generation in Zebrafish Blood
}

\author{
Evelien Schurgers*, Martijn Moorlag*, H. Coenraad Hemker, Theo Lindhout, \\ Hilde Kelchtermans, Bas de Laat \\ * Both authors contributed equally to this work.
}

PlosOne, 2016, e0149135 


\section{Abstract}

Background To better understand hypercoagulability as an underlying cause for thrombosis, the leading cause of death in the Western world, new assays to study ex vivo coagulation are essential. The zebrafish is generally accepted as a good model for human haemostasis and thrombosis, as the haemostatic system proved to be similar to that in man. Their small size however, has been a hurdle for more widespread use in haemostasis related research.

Methods In this study we developed a method that enables the measurement of thrombin generation in a single drop of non-anticoagulated zebrafish blood. Scanning electron microscopy for the visualization of the fibrin network.

Results Pre-treatment of the fish with inhibitors of FXa and thrombin, resulted in a dose dependent diminishing of thrombin generation, demonstrating the validity of the assay. In order to establish the relationship between whole blood thrombin generation and fibrin formation, we visualized the resulting fibrin network by scanning electron microscopy.

Conclusion Taken together, in this study we developed a fast and reliable method to measure thrombin generation in whole blood collected from a single zebrafish. Given the similarities between coagulation pathways of zebrafish and mammalians, zebrafish may be an ideal animal model to determine the effect of novel therapeutics on thrombin generation. Additionally, because of the ease with which gene functions can be silenced, zebrafish may serve as a model organism for mechanistical research in thrombosis and haemostasis. 


\section{Introduction}

Thrombosis remains a leading cause of death in the western world. Aside from mortality, significant morbidity occurs from thrombotic events. The causes of this hypercoagulability are becoming more and more clear with an enhanced knowledge of haemostasis and the development of new coagulation assays. Most of this knowledge results from extensive in vitro biochemical characterization of blood coagulation, whereas studies investigating blood coagulation in vivo are limited.

Due to the availability of knockout technology, genetic studies of thrombosis in mice are popular. Nonetheless, the time consuming and labour-intensive process of generating knockouts restricts these studies. The zebrafish is generally accepted as a good model for mammalian haemostasis and thrombosis due to the presence of coagulation factors, platelet receptors and its response to anticoagulant drugs commonly used in clinical treatment ${ }^{1}$. In addition, haemostatic pathways in zebrafish proved to be similar to those in $\operatorname{man}^{2-4}$. Interestingly, the use of zebrafish enables large scale mutagenesis screening to identify novel genes involved in haemostasis and thrombosis ${ }^{5,6}$.

The small size of zebrafish has been a hurdle in thrombosis and haemostasis research since most of the conventional coagulation assays require large amounts of plasma. Jagadeeswaran et al. optimized a total coagulation activity screening assay using small quantities of zebrafish plasma, by adding human fibrinogen and measuring fibrin formation by turbidimetry to probe thrombin formation ${ }^{7}$. Additionally, they developed an ultra-sensitive kinetic method to identify specific pathway defects in small quantities of plasma. Disadvantages of such assays include the requirement of multiple zebrafish and the use of plasma, not considering the effect of thrombocytes and erythrocytes on coagulation.

At present, mainly end-point assays are used to detect coagulation defects. These assays simply measure the time it takes for a platelet-poor plasma sample to clot, i.e. when the first traces of fibrin are formed. However, fibrin formation already starts in the presence of tiny amounts of thrombin $(\approx 1 \mathrm{nM})^{8}$. Thus, the vast majority of thrombin takes place after fibrin formation ${ }^{9}$, suggesting that clotting time-based assays only measure the initiation and not the propagation phase of coagulation. Importantly, correct functioning of the haemostatic system proved to be dependent on the total amount of thrombin that is formed during coagulation ${ }^{10}$. We recently developed a reliable method to measure thrombin generation in a drop of whole blood, thereby bringing coagulation one step closer to physiology ${ }^{11}$. In this study, we further optimized this method enabling the determination of thrombin generation in a drop of non-anticoagulated whole 
blood obtained from a single zebrafish. Thrombin generation proved to be sensitive to pre-treatment of the fish with inhibitors of FXa and thrombin. Furthermore, we visualized the resulting fibrin network by scanning electron microscopy (SEM) in order to analyse the density and dimensions of the fibrin strands.

\section{Materials and methods}

\section{Reagents}

$20 \mathrm{mM}$ Hepes buffer ( $\mathrm{pH}$ 7.35) containing $5 \mathrm{mg} / \mathrm{ml}$ bovine serum albumin (BSA) and $140 \mathrm{mM} \mathrm{NaCl}$ (BSA5) or $60 \mathrm{mg} / \mathrm{ml}$ bovine serum albumin (BSA60) were prepared as described previously ${ }^{12}$. The rhodamine-based substrate $P_{2} R$ ho was a kind gift of Diagnostica Stago. The calibrator, $\alpha_{2}$ macroglobulin-thrombin $\left(\alpha_{2} M-T\right)$ complex was prepared in-house as described previously ${ }^{12}$. Rivaroxaban (Xarelto) was from Bayer and melagatran was a gift from AstraZeneca.

\section{Blood collection and treatment of zebrafish}

This study was carried out in strict accordance with the recommendations in the guide for the use of laboratory animals of the university of Liège. The protocol was approved by the committee on the ethics of animal experiments of the university of Liege Permit number LA 1610002. Blood was collected from adult (male and female) wild type zebrafish (Danio rerio) as described previously ${ }^{7}$. Briefly, with a small pair of scissors, an incision was made at the lateral side of the fish just posterior of the dorsal fin, thereby transecting the dorsal vein/artery. From the blood welling up in the wound, $5 \mu$ l was collected for further analysis.

For anticoagulant treatment, fish were sedated with a high dose of tricaine solution $(1.6 \mathrm{mg} / \mathrm{ml})$. Fish were dried with paper and weighed. Only fish weighing less than 1 gram were used and injected intraperitoneally with $20 \mu \mathrm{l} / \mathrm{g}$ of the indicated anticoagulant in phosphate buffered saline (PBS) and $0.25 \%$ phenol red (to monitor the injection process). After injection, fish were allowed to recover for 30 minutes after which blood was collected as described above.

\section{Thrombin generation measurement}

For thrombin generation, an adapted protocol was developed based on our whole blood thrombin generation assay ${ }^{11}$. Collected whole blood $(5 \mu \mathrm{l})$ was mixed with 5 $\mu l$ of HEPES buffer containing the $P_{2}$ Rho substrate (final concentration $(\mathrm{fc}) 300$ $\mu \mathrm{M}) .5 \mu \mathrm{l}$ of this mixture was put on a paper disk and covered with mineral oil to prevent evaporation. The lag-time phase of the thrombin generation experiment was started as soon as the incision for the blood withdrawal was made. Calibration was done by adding $5 \mu \mathrm{l}$ of whole blood to $5 \mu \mathrm{l}$ of HEPES buffer containing $\mathrm{P}_{2} \mathrm{Rho}$ (fc $300 \mu \mathrm{M}$ ), a2M-thrombin calibrator (fc $100 \mathrm{nM}$ ) and citrate (fc 9,8 mM). 
Fluorescence was recorded with a fluorescence detector (ESElog, Qiagen) with $\lambda_{\text {ex }}$ $=485 \mathrm{~nm}$ and $\lambda_{\mathrm{em}}=538 \mathrm{~nm}$. All experiments were performed at $37^{\circ} \mathrm{C}$.

Analysis of the fluorescence tracings to yield the thrombogram and corresponding parameters was performed with a modified method, considering only the thrombin generation until the peak is reached. From the resulting thrombogram the following parameters were calculated: lagtime ( $\mathrm{min}$ ), peak (nM, maximal thrombin concentration), peak-endogenous thrombin potential (ETP, nM.min, area under the thrombin curve until the peak is reached), time to peak and velocity ( $\mathrm{nM} / \mathrm{min}$, maximal rate of thrombin generation).

The human samples were analysed using the plasma CAT as previously described ${ }^{12}$ in the presence or absence of either rivaroxaban or melagatran.

\section{Plasma samples for normal pool plasma}

After approval of the local medical ethical board (Medical Ethical Committee of Maastricht University Medical Centre) 24 healthy adult volunteers who did not take any drugs for at least two weeks gave full informed written consent according to the Helsinki declaration. Blood was collected aseptically by antecubital puncture into vacuum tubes ( 1 volume trisodium citrate $0.105 \mathrm{M}$ to 9 volumes blood) (BD Vacutainer System).

For the normal pooled plasma (NPP), blood from the 24 volunteers was prepared by centrifuging the blood at $2900 \mathrm{~g}$ during $10 \mathrm{~min}$ at room temperature. Plasma was aspirated and the procedure was repeated. Plasmas were pooled and further ultra-centrifugation (100000g, $70 \mathrm{~min}$ ) was carried out. Aliquots of $1 \mathrm{ml}$ were stored at $-80^{\circ} \mathrm{C}$ until use.

\section{SEM analysis}

After thrombin generation was determined in whole blood, the clots were prepared for visualization by SEM. The clots were fixated by adding $2.5 \%$ glutaraldehyde (grade I, Sigma Aldrich, St. Louis, Missouri) in PBS (Sorensen's, pH 7.2) (Electron Microscopy Sciences, Hatfield, PA, USA) for 1 hour at room temperature and then placing it at $4^{\circ} \mathrm{C}$ overnight. The following day, the glutaraldehyde solution was removed and the samples were repeatedly $(5 x)$ washed with PBS. As a secondary fixation, the samples were placed in osmiumtetroxide $\left(\mathrm{OsO}_{4}, 1 \%\right)$ diluted in sodium cacodylate $(200 \mathrm{mM}, \mathrm{pH}$ 7.4) (Electron Microscopy Sciences, Hatfield, PA, USA) for 1 hour at room temperature. Consecutively, the clots were dehydrated in ethanol (30\%,50\%, 70\%, 90\% and 3 times at 100\%) for 3 minutes. The samples were then treated with a hexamethyldisilane/ethanol solution for 3 minutes and in hexamethyldisilane (Sigma Aldrich, St. Louis, MO, USA) for 10 minutes. The samples were removed 
Chapter 3

from the wells, left to dry and coated with gold. Analysis was performed on a desktop SEM (Phenom-World, Eindhoven, the Netherlands). 


\section{Statistical analysis}

For statistical analysis of the data GraphPad Prism Software was used. Differences between two groups were evaluated by the one-way ANOVA test or the Mann Whitney $U$ test.

\section{Results}

Development of an assay to measure thrombin generation in zebrafish

In a first set of experiments, thrombin generation was determined in $5 \mu$ whole blood collected from single zebrafish, using a method comparable to whole blood calibrated automated thrombography ${ }^{11}$. The limited blood volume of a single zebrafish, impedes the performance of both a thrombin generation and calibrator measurement. Since calibrator measurements performed on the blood of 28 different fish in 5 independent experiments demonstrated an acceptable variation (mean CV of 14\%), the average calibrator slope per experiment was used for thrombin generation calculations.

Subsequently, thrombin generation was measured in 5 independent experiments, each consisting of 3 to 5 thrombin generation measurements. Thrombin generation curves, together with the average and \%CV of the individual thrombin generation parameters are shown in figure 1 . The thrombin enzyme from zebrafish clearly proved to be capable of cleaving our rhodamine-based substrate. Interestingly, thrombin generation measured in individual fish showed a similar amount of inter-individual variation as in humans ${ }^{11}$. Striking differences with human thrombin generation include the much shorter lag-time, and the increased peak height and velocity index. Taken together, our results demonstrate the feasibility of measuring thrombin generation in whole blood collected from a single zebrafish. 


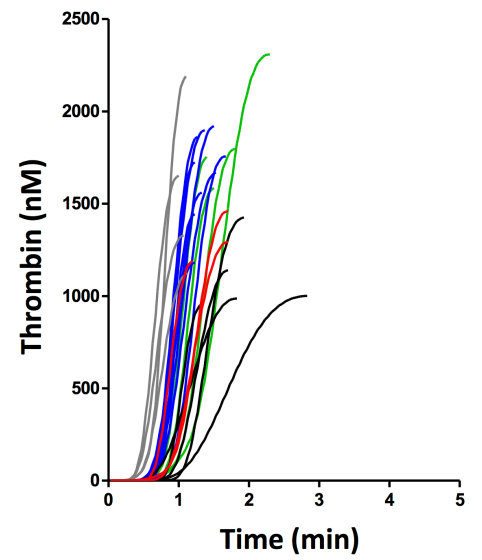

\begin{tabular}{lcc}
\hline & Mean & Mean CV (\%) \\
\hline Peak ETP (nM.min) & 707.46 & 33.78 \\
Peak (nM) & 1504.00 & 17.75 \\
Lag time (min) & 0.50 & 13.05 \\
Time to peak (min) & 1.53 & 17.51 \\
Velocity (nM/min) & 3197.60 & 26.14 \\
\hline
\end{tabular}

Figure 1. Thrombin generation curves and parameters in zebrafish.

Thrombin generation was measured in 5 independent experiments, in a total of 25 zebrafish. (A) Thrombin generation curves are shown. Each colour represents an independent experiment. (B) Mean thrombin generation parameters and their $\mathrm{CV}$ are calculated.

In a next set of experiments, the effect of pre-treatment of the zebrafish with thrombin or FXa inhibitors on thrombin generation parameters was tested. Zebrafish were treated with the indicated compounds and doses for 30 minutes as mentioned before, followed by blood collection and thrombin generation measurements. Thrombin generation curves of the individual zebrafish are shown in figure 2. Results are expressed as the percentage inhibition compared to a control group of vehicle-treated fish $(n=8)$ and are representative for 2 independent experiments. 
A

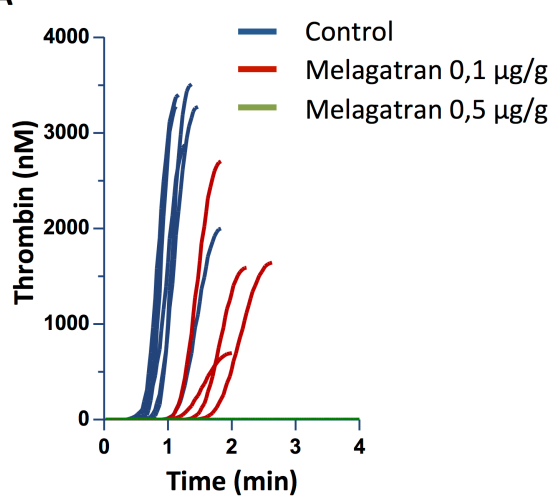

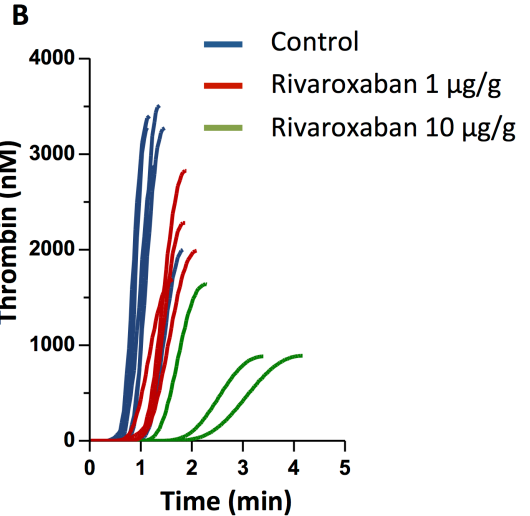

C

\begin{tabular}{lcccccc}
\hline & \multicolumn{2}{c}{$\begin{array}{c}\text { Melagatran } \\
(\mathbf{0 . 1} \boldsymbol{\mu g} / \mathbf{g} ; \mathbf{n}=\mathbf{4})\end{array}$} & \multicolumn{2}{c}{$\begin{array}{c}\text { Rivaroxaban } \\
(\mathbf{1} \boldsymbol{\mu g} / \mathbf{g} ; \mathbf{n}=\mathbf{4})\end{array}$} & \multicolumn{2}{c}{$\begin{array}{c}\text { Rivaroxaban } \\
(\mathbf{1 0} \boldsymbol{\mu g} / \mathbf{g} ; \mathbf{n}=\mathbf{3})\end{array}$} \\
\hline & \% inhibition & $\mathbf{P}$-value* & \% inhibition & P-value & \% inhibition & P-value $^{\text {s }}$ \\
\hline Peak ETP (nM.min) & 27.28 & $\mathrm{~ns}$ & -8.18 & $\mathrm{~ns}$ & 11.13 & $\mathrm{~ns}$ \\
Peak (nM) & 46.58 & $\mathbf{0 . 0 1}$ & 29.25 & $\mathrm{~ns}$ & 63.36 & $<\mathbf{0 . 0 1}$ \\
Lag time (min) & -102.03 & $\mathbf{0 . 0 1}$ & -18.09 & $\mathrm{~ns}$ & -150.41 & $<\mathbf{0 . 0 5}$ \\
Time to peak (min) & -61.40 & $\mathbf{0 . 0 1}$ & -39.13 & $\mathrm{~ns}$ & -143.23 & $<\mathbf{0 . 0 1}$ \\
Velocity (nM/min) & 64.25 & $\mathbf{0 . 0 1}$ & 56.93 & $\mathrm{~ns}$ & 84.97 & $<\mathbf{0 . 0 1}$ \\
\hline
\end{tabular}

Figure 2. Inhibition of thrombin generation in zebrafish by thrombin or FXa inhibition.

Zebrafish were treated with Melagatran (A) or rivaroxaban (B) at the indicated doses for $30 \mathrm{~min}$, followed by thrombin generation measurements. (C) Results are expressed as the percentage inhibition compared to a control group of vehicle-treated fish $(n=4)$. Results are representative for two independent experiments. Statistical differences between treatment and control groups were performed with *Mann-Whitney U-test or \$1-way ANOVA. (ns = not significant)

As to the thrombin inhibition, treatment with melagatran concentrations above 0.5 $\mu \mathrm{g} / \mathrm{g}$ completely blocked thrombin generation, clearly illustrating that the observed fluorescent signal is thrombin- related. A concentration of $0.1 \mu \mathrm{g} / \mathrm{g}$ melagatran significantly inhibited thrombin generation, as evident from the significantly decreased peak height and reduced lag-time, time to peak and velocity index. The dosage needed to inhibit thrombin generation in zebrafish was considerably lower than concentrations used in in vitro experiments with normal pooled plasma. In these experiments a concentration range of 1073 to $3575 \mu \mathrm{g} / \mathrm{g}$ was needed to get a dose dependent inhibition of thrombin generation. Comparable results were obtained upon injection of another thrombin inhibitor, hirudin $(4 \mu \mathrm{g} / \mathrm{g})$ (data not shown). Additionally, inhibition of FXa by rivaroxaban 
treatment (concentrations of 1 and $10 \mu \mathrm{g} / \mathrm{g}$ ) resulted in a similar inhibition of the thrombin generation. The concentration was in contrast with the in vitro results in normal pooled plasma, in which lower concentrations from $72,65 \mu \mathrm{g} / \mathrm{g}$ rivaroxaban already resulted in inhibition of thrombin generation. However, the dosages used in the zebrafish were much closer to the prescribed dose for adults of $20 \mathrm{mg} / \mathrm{day}$ $( \pm 0.29 \mu \mathrm{g} / \mathrm{g})$

\section{SEM analysis of fibrin clots}

Given the accelerated and increased thrombin generation in zebrafish compared to humans, we decided to analyse the fibrin ultrastructure at the end of a thrombin generation experiment by SEM. The mineral oil that was used to prevent evaporation was removed and the fibrin that was formed was fixated for visualization with SEM. A representative image of the fibrin network is shown in figure 3. Analysis of the clot revealed a much denser network compared to humans, composed of thin fibrin fibres. Interestingly, the structure of the network is comparable to a human fibrin network triggered with high tissue factor concentrations (data not shown). The entrapped red blood cells and thrombocytes in the fibrin network, might suggest their participation in the process of coagulation.

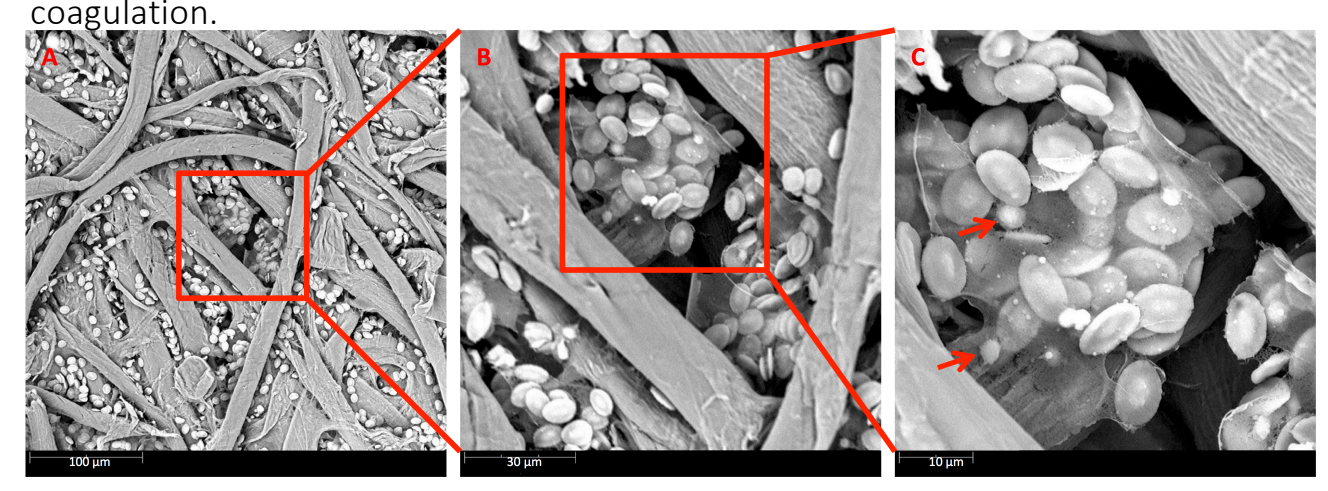

Figure 3. Scanning electron microscopy (SEM) of zebrafish blood clots. (A)

Representative image of SEM analysis of blood clots formed during thrombin generation measurements. (B-C) Sequential enlargements of the fibrin network with entrapped red blood cells. Arrows in (C) indicate platelets.

\section{Discussion}

In this study, we optimized an assay to measure thrombin generation in whole blood obtained from a single zebrafish. A major advantage compared to previous tests, is the use of whole blood instead of plasma, resulting in a quick and reliable screening tool to measure blood coagulation in zebrafish. Furthermore, as the 
measurement starts directly after the blood collection, the use of an anticoagulant that may influence coagulation is avoided.

The suitability of the zebrafish model to study haemostasis depends hugely on the degree of similarity between the zebrafish and mammalian systems. Previous studies have established that, despite the evolutionary distance, the major coagulant and anticoagulant pathways are similar between zebrafish and mammalians, as evident from the presence of a comparable contact activation system, extrinsic pathway and common pathway ${ }^{3}$. The effects of prothrombin deficiency in zebrafish embryos, ranging from early morphological defects and internal bleeding to occasional bleeding in the brain at a later stage, suggest that the mechanism of thrombin signalling is conserved across vertebrates ${ }^{13}$. To our knowledge, our method is the first that allows measurement of thrombin generation in whole blood obtained from a single zebrafish. Thrombin generation experiments in zebrafish showed a similar inter-individual variation as in humans ${ }^{11}$. However, thrombin generation proved to be significantly accelerated and increased compared to humans. With the current blood collection method, using a pair of scissors, exposure of the blood to tissue factor is inevitable. Therefore, the current method is suits well for tests related to the intrinsic coagulation system.

By pre-treating zebrafish with thrombin inhibitors, we clearly provided evidence that the observed fluorescent signals in our assay are the result of thrombin. The treatment with thrombin inhibitors, hirudin and FXa was found to significantly inhibit thrombin generation in zebrafish. Hirudin microinjections into early zebrafish embryos were previously shown to inhibit fibrin forming activity and to cause abnormal development, suggesting a role for thrombin in early development ${ }^{14}$. Furthermore, Jagadeeswaran et al. demonstrated that warfarin has similar effects in zebrafish compared to mammals, illustrating that vitamin $\mathrm{K}$ dependent pathways in fish are comparable to human pathways ${ }^{3,7}$. Interestingly, as all tested anticoagulant therapy was found to diminish thrombin generation in zebrafish, zebrafish may be used as an in vivo model system to test the pharmacokinetic and pharmacodynamic aspects of (novel) therapeutics on thrombin generation.

As to the fibrin formation, the appearance of a dense fibrin network composed of thin fibrin fibres is in line with the accelerated and increased thrombin generation. Indeed, high versus low concentrations of thrombin lead to dense networks of thin fibres versus permeable clots composed of thick woven fibrin strands, respectively ${ }^{8,15,16}$.

In conclusion, we developed a fast and reliable method to measure thrombin generation in whole blood collected from a single zebrafish. Given the huge 
similarities between coagulation pathways of zebrafish and mammalians, zebrafish may be an ideal in vivo model to determine the effect of novel therapeutics on thrombin generation. Additionally, because of the ease with which gene functions can be silenced, zebrafish may serve as a model organism for further mechanistical research in thrombosis and haemostasis.

\section{Acknowledgments}

We thank Prof. P. Jagadeeswaran to teach us the method of blood collection. We are grateful for the excellent collaboration with Dr. M. Winandy and Dr. H. Pendeville for zebrafish housing and care. We thank D. Bergemann for instructions on intraperitoneal injections. 


\section{References}

1. $\quad$ Carradice, D. \& Lieschke, G. J. Zebrafish in hematology: sushi or science? Blood 111, 33313342 (2008).

2. Sheehan, J. et al. Demonstration of the extrinsic coagulation pathway in teleostei: Identification of zebrafish coagulation factor VII. Proc. Natl. Acad. Sci. 98, 8768-8773 (2001).

3. Jagadeeswaran, P. \& Sheehan, J. P. Analysis of Blood Coagulation in the Zebrafish. Blood Cells. Mol. Dis. 25, 239-249 (1999).

4. Jagadeeswaran, P., Sheehan, J. P., Craig, F. E. \& Troyer, D. Identification and characterization of zebrafish thrombocytes. Br. J. Haematol. 107, 731-738 (1999).

5. Driever, W. et al. A genetic screen for mutations affecting embryogenesis in zebrafish. Development 123, 37-46 (1996).

6. Nasevicius, A. \& Ekker, S. C. Effective targeted gene 'knockdown' in zebrafish. Nat. Genet. 26, 216-220 (2000).

7. Jagadeeswaran, P., Gregory, M., Johnson, S. \& Thankavel, B. Haemostatic screening and identification of zebrafish mutants with coagulation pathway defects: an approach to identifying novel haemostatic genes in man. Br. J. Haematol. 110, 946-956 (2000).

8. Wolberg, A. S. \& Campbell, R. A. Thrombin generation, fibrin clot formation and hemostasis. Transfus. Apher. Sci. 38, 15-23 (2008).

9. Hemker, H. C., Dieri, A., Raed, Smedt, E. D. \& Beguin, S. Thrombin generation, a function test of the haemostaticthrombotic system. Thromb. Haemost. 96, 553-561 (2006).

10. Al Dieri, R., de Laat, B. \& Hemker, H. C. Thrombin generation: What have we learned? Blood Rev. 26, 197-203 (2012).

11. Ninivaggi, M. et al. Whole-Blood Thrombin Generation Monitored with a Calibrated Automated Thrombogram-Based Assay. Clin. Chem. 58, 1252-1259 (2012).

12. Hemker, H. C. et al. Calibrated Automated Thrombin Generation Measurement in Clotting Plasma. Pathophysiol. Haemost. Thromb. 33, 4-15 (2003).

13. Day, K., Krishnegowda, N. \& Jagadeeswaran, P. Knockdown of prothrombin in zebrafish. Blood Cells. Mol. Dis. 32, 191-198 (2004).

14. Jagadeeswaran, P., Liu, Y. C. \& Eddy, C. A. Effects of Hirudin (Thrombin Specific Inhibitor) in Zebrafish Embryos: A Developmental Role for Thrombin. Blood Cells. Mol. Dis. 23, 410-414 (1997).

15. Shah, G. A., Nair, C. H. \& Dhall, D. P. Physiological studies on fibrin network structure. Thromb. Res. 40, 181-188 (1985).

16. Ryan, E. A., Mockros, L. F., Weisel, J. W. \& Lorand, L. Structural origins of fibrin clot rheology. Biophys. J. 77, 2813-2826 (1999). 


Chapter 4

\section{On the Causes of Inter-Laboratory Variability in Calibrated Thrombin Generation Measurement.}

Martijn Moorlag, Konstantin Guria, Bas de Laat, H. Coenraad Hemker, Julien Perrin 


\section{Abstract}

Background The thrombin generation capacity of plasma is an important determinant of the individual thrombotic or bleeding tendency, which makes it a promising clinical variable. At this moment however, its implementation in routine clinical practice is hindered by a relatively high variability between different laboratories. Efforts to improve this variability by normalisation have improved the comparability of some parameters and yet worsened others.

Methods we analysed 34 sets of fluorescence curves of the same sample of reference plasma that were obtained under "real life" conditions in 12 different clinical laboratories throughout France, Belgium and Switzerland.

Results Our analysis revealed two major sources of inter-laboratory variability: Errors due to the calibration procedure and errors due to insufficient temperature control. About $30 \%$ of experimental variation can be attributed to the former and about $20 \%$ to the latter.

Conclusions Elimination of these errors reduces the total inter-laboratory variability of the area under the thrombin generation curve from 16.3 to $8.1 \%$ and that of the peak thrombin activity measured from 17.4 to $8.6 \%$. 


\section{Introduction}

\section{Thrombin generation, potential benefits of its introduction into clinical practice}

Measuring the thrombin level in clotting plasma is an established tool in blood coagulation research since around 1950 but the technique was too laborious for routine clinical use $\mathrm{e}^{1,2}$. The evolution of the method to the continuous measurement of thrombin by its cleavage of an added fluorogenic substrate increased throughput to around 24 samples per hour ${ }^{3,4}$. The increased throughput triggered a widespread interest in this approach. Throughout the last decades considerable evidence has been published that the amount of thrombin activity that develops in a sample of clotting blood(-plasma) is an indicator of the risk of bleeding ${ }^{5-8}$ as well as venous thrombosis ${ }^{9-17}$. It also relates to the risk of arterial thrombosis, be it in a less straightforward manner ${ }^{18-20}$. It is a common denominator of the effect of all anticoagulants ${ }^{21-28}$ and, when it is measured in platelet rich plasma, it is inhibited by platelet inhibitors ${ }^{29,30}$. Therefore, it has a promising outlook for use as a clinical parameter.

In calibrated automated thrombography (CAT) the amount of thrombin in the sample in which thrombin is generating (the staple sample), is calculated by comparison to the fluorescence that develops in a separate sample of the same plasma in which a constant known amount of thrombin activity converts the substrate (the calibrator sample). In this way, the value of the calibration factor (CF) can be read at every level of fluorescence. The same plasma needs to be used in both the thrombin generation (TG) and the calibrator experiment because e.g. the colour of individual plasmas influences fluorescence in a different way.

Inter-laboratory variability as an obstacle for clinical implementation The main issue preventing broad clinical use of TG is the relatively high interlaboratory variability of its results ${ }^{31}$. This has been attributed to a lack of automation and because of its sensitivity to pre-analytical variables ${ }^{32}$. Rigorous standardization of the pre-analytical and analytical procedure and normalization relative to a reference plasma indeed improved the $\mathrm{CV}^{33,34}$. A multicentre study from French-speaking countries (France, Belgium, Switzerland) carried out under "real life" conditions in clinical and research laboratories showed that the use of a common reference plasma could improve inter-laboratory variability of most TG parameters except in cases of frank hypo-coagulability ${ }^{35}$. In the present work, we utilized the original fluorescence traces from the common reference plasma used in this study to investigate possible causes of the observed variability.

It has been observed that TG is "inversely" temperature sensitive: the lower the temperature, the higher the thrombin activity found ${ }^{36}$. Consequently, the TG test is 
critically dependent upon the preheating of the samples: insufficient preheating makes the temperature rise during the first minutes of the measurements, leading to an underestimation of the calibrator activity, hence causing an overestimation of the CF, and finally resulting in an underestimation of the thrombin concentration. Moreover, the temperature equilibration process largely coincides with the lag-time in the test sample, and thus is certain to influence this variable. In so far as it extends beyond the lag-time, insufficient temperature control will influence the course of TG as well. The combination of underestimation due to delayed heating of the calibrator and enhancement of TG due to delayed heating of the test sample will result in unpredictable overestimation of the recorded values of Endogenous Thrombin Potential (ETP) and Peak.

The aim of the current study was to evaluate the sources of inter-laboratory variation and to quantify the sources of the variability, notably temperature. Moreover, its aim is to propose the most appropriate strategy for improvement of the assay.

\section{Materials and methods}

\section{Data set description}

Graphs of fluorescence (in arbitrary units (AU)) against time of both calibrator curves and TG curves were kindly provided by the labs that partook in the study of Perrin $^{35}$. In total we obtained the data of 34 separate experiments in which samples of the same reference plasma were measured in 12 different labs on different days. A detailed description of the experimental conditions and protocol can be found in the original article ${ }^{35}$. In short, a unique and common normal reference plasma (reference Plasma Stago ${ }^{\circledR}$, Diagnostica Stago Asnières) was used in a standard TG experiment at 5pM tissue factor (TF) in 34 separate experiments performed in 12 laboratories. In the calibration experiments the $\alpha 2$ macroglobulinthrombin complex ( $\alpha 2 \mathrm{M}-\mathrm{T})$, Thrombin Calibrator ${ }^{\mathrm{TM}}$ (Diagnostica Stago Asnières)) was added as the equivalent of constant thrombin activity. The different participating laboratories used different batches of calibrator. No instruction was given as to preheating of the plates prior to the measurement. The mean values, standard deviations and variation coefficients of thrombin generation parameters for initial data set can be found in table 1. 
Table 1. Mean values, standard deviations and variation coefficients of TG parameters for initial data set.

\begin{tabular}{|l|l|l|l|l|}
\hline & $\begin{array}{l}\text { ETP, } \\
\mathrm{nM} \cdot \mathrm{min}\end{array}$ & Peak, nM & $\begin{array}{l}\text { ttpeak, } \\
\text { min }\end{array}$ & Lagtime, min \\
\hline Mean & 1553.7 & 268.0 & 5.90 & 2.97 \\
\hline $\begin{array}{l}\text { Standard } \\
\text { deviation }\end{array}$ & 252.8 & 46.5 & 0.48 & 0.35 \\
\hline $\begin{array}{l}\text { Variation } \\
\text { coefficient, \% }\end{array}$ & 16.3 & 17.4 & 8.2 & 11.7 \\
\hline
\end{tabular}

\section{Temperature control estimation}

The stability of the temperature throughout the experiment was assessed from the raw fluorescent data of the calibration curves by means of a specially designed program written by us in Mathematica 10.0 (Wolfram Research). The code of the program is provided in Supplementary materials. Its algorithm is based on the use of the graph of the first derivative of fluorescence $(\mathrm{dF} / \mathrm{dt})$ against the fluorescence $(F)$ itself previously defined as the "diagnostic plot" ${ }^{\prime 7,38}$. At constant temperature $\mathrm{dF} / \mathrm{dt}$ is highest at zero time $\left(\mathrm{F}_{0}\right)$ because substrate concentration is highest and the inner filter effect is still absent (figure $1 \mathrm{~A}$ ). An increase of $\mathrm{dF} / \mathrm{dt}$ in the beginning of the experiment can only be attributed to an increase of temperature. In case of a constant temperature, the shape of the diagnostic plot could be described by a decreasing branch of a parabola (for the rationale behind this see $\left.\operatorname{ref}^{37}\right)$.
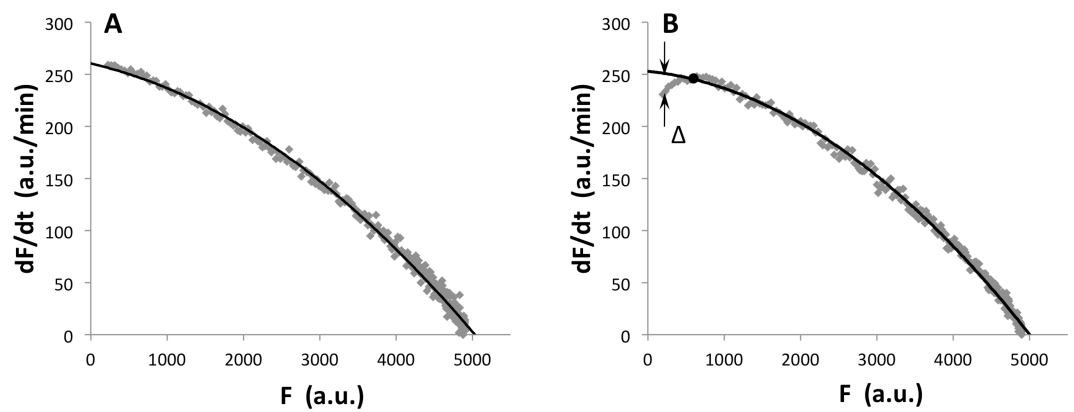

Figure 1. Diagnostic plots for the calibration curves measured after 15 min of preheating (A) and after 2 min of preheating (B).

Bold black point in section (B) corresponds to the end point of the "shaft" of the hockey stick. $\Delta$ is the difference between measured initial value of $\mathrm{dF} / \mathrm{dt}$ and the value obtained from the extrapolation of the "shaft" to $F_{0}$.

The "hockey stick" is not a very precise metaphor here as the "shaft" is not straight, but we will still use it for the sake of visualization of the algorithm used for the evaluation of the magnitude of temperature changes. 
When preheating of the samples is insufficient, it results in diagnostic plots with a characteristic "hockey stick" appearance (figure 1B). The initial uprising part of such a curve, representing the temperature relaxation process, corresponds to the "head" of the "hockey stick" and the descending part corresponds to its "shaft". The "hockey stick" is not a very precise metaphor here as the "shaft" is not straight, but we will still use it for the sake of visualization of the algorithm used for the evaluation of the magnitude of temperature changes.

For each diagnostic plot its part unperturbed by the temperature relaxation processes, i.e. the "shaft" of the hockey stick. The program selected the longest possible "shaft" on the diagnostic plot basing on the criteria that its initial point should lie inside standard deviation range of the residuals of a parabolic approximation fitted to the "shaft". Afterwards the parabolic approximation of the "shaft" was extrapolated backwards to the initial zero time point to calculate the difference $(\Delta)$ between measured initial value of $\mathrm{dF} / \mathrm{dt}$ and the value obtained from the shaft's approximation (figure 2B). As an estimate of the temperature error we used the increase of the $\mathrm{CF}\left(\mathrm{CF}_{\text {inc }}\right)$ at the zero-time point calculated as the ratio of $\Delta$ to the value of $\mathrm{dF} / \mathrm{dt}$ measured at the beginning of the experiment:

$\mathrm{CF}_{\text {inc }}=\frac{\Delta}{\left.\frac{\mathrm{dF}}{\mathrm{dt}}\right|_{\mathrm{t}=0}}$

Finally, for each set of TG curves measured in the same plate the quality of temperature control was estimated by the averaged value of $C F_{\text {inc }}$ calculated for all calibration curves present in that particular plate.

\section{Evaluation of errors induced by calibration}

During a TG experiment $\alpha 2 \mathrm{M}-\mathrm{T}$ forms from the interaction of the inhibitor $\alpha 2$ macroglobulin $(\alpha 2 \mathrm{M})$ with thrombin. $\alpha 2 \mathrm{M}$ is present in sufficient excess over thrombin, $\sim 3$ vs. $0.3 \mu \mathrm{M}$ respectively to safely assume that the reaction is first order. The amount of $\alpha 2 \mathrm{M}-\mathrm{T}$ formed is therefore directly proportional to the amount of thrombin and to the time it is active i.e. the final $\alpha 2 \mathrm{M}-\mathrm{T}$ concentration is proportional to the ETP. The ratio of the $\alpha 2 \mathrm{M}-\mathrm{T}$ end level to the ETP therefore is in theory constant and should be independent of any error that could be introduced by calibration.

We used this fact to remove the effect of possible calibrator activity errors. To this end we used the values of $\alpha 2 \mathrm{M}-\mathrm{T}_{\text {end }}$ as calculated by the Thrombinoscope ${ }^{\circledR}$ software to compare the individual $\alpha 2 \mathrm{M}-\mathrm{T}_{\text {end }}$ value to the average of all $\alpha 2 \mathrm{M}-\mathrm{T}_{\text {end }}$ values from the experimental data from the different laboratories combined. This comparison yielded a correction factor $(R)$ that was applied to calculate an internally calibrated ETP $\left(\mathrm{ETP}_{\text {ic }}\right)$. So: $\mathrm{ETP}_{\text {ic }}=\mathrm{R} \cdot \mathrm{ETP}_{\mathrm{exp}}$ where $\mathrm{R}=\left(\right.$ Average $\left.\alpha 2 \mathrm{M}-\mathrm{T}_{\text {end }}\right) /$ 
(Experimental $\left.\alpha 2 \mathrm{M}-\mathrm{T}_{\text {end }}\right)$. An analogous formula was used to calculate "internally calibrated Peak".

\section{Results}

\section{Continuous calibration, calculation of free thrombin}

As explained under methods, it is possible to correct for calculation errors in the ETP via the ratio between the ETP and the end-level of $\alpha 2 \mathrm{M}-\mathrm{T}$, because the proportion, to which ETP is over- or under-estimated by the calibration procedure, is the same as the proportion to which the final level of $\alpha 2 \mathrm{M}-\mathrm{T}$ is under- or overestimated. This property we used to correct the values of ETP and peak as described under methods. In table 2 it is seen in how far the experimental error is diminished by this procedure. In fact, the CV for the internally calibrated ETP is reduced by almost $30 \%$ as compared with the initial CV with a reduction from $16,3 \%$ to $11,7 \%$. A similar trend is observed for the thrombin peak in which the inter-laboratory CV is reduced from $17,4 \%$ to $11,9 \%$. The remaining error must be the experimental error in the curve of amidolytic activity, $f_{a}(t)$, which includes the error due to insufficient temperature equilibration and possible errors in the calculation of ETP and $\alpha 2 \mathrm{M}-\mathrm{T}$ from the raw data ${ }^{38}$.

\section{Errors due to insufficient temperature equilibration}

For each experiment, the degree of the temperature control quality $\mathrm{CF}_{\text {inc }}$ was calculated. The corrected values of ETP and Peak for all 34 experiments are plotted against $\mathrm{CF}_{\text {inc }}$ (figure $2 \mathrm{C}, \mathrm{D}$ ). The higher the temperature jump in the beginning, the lower the actual temperature was during the experiment. TG increases when the temperature decreases as can be seen when compared to figure $2 \mathrm{~A}, \mathrm{~B}$; it is therefore not surprising to find higher TG values in the cases where there was a higher temperature jump. Figure 2 confirms this relation between TG parameters and $\mathrm{CF}_{\text {inc }}$. Moreover, this relation allowed us to subtract a temperature dependent trend from the data and evaluate the variability of the data after this second correction. 

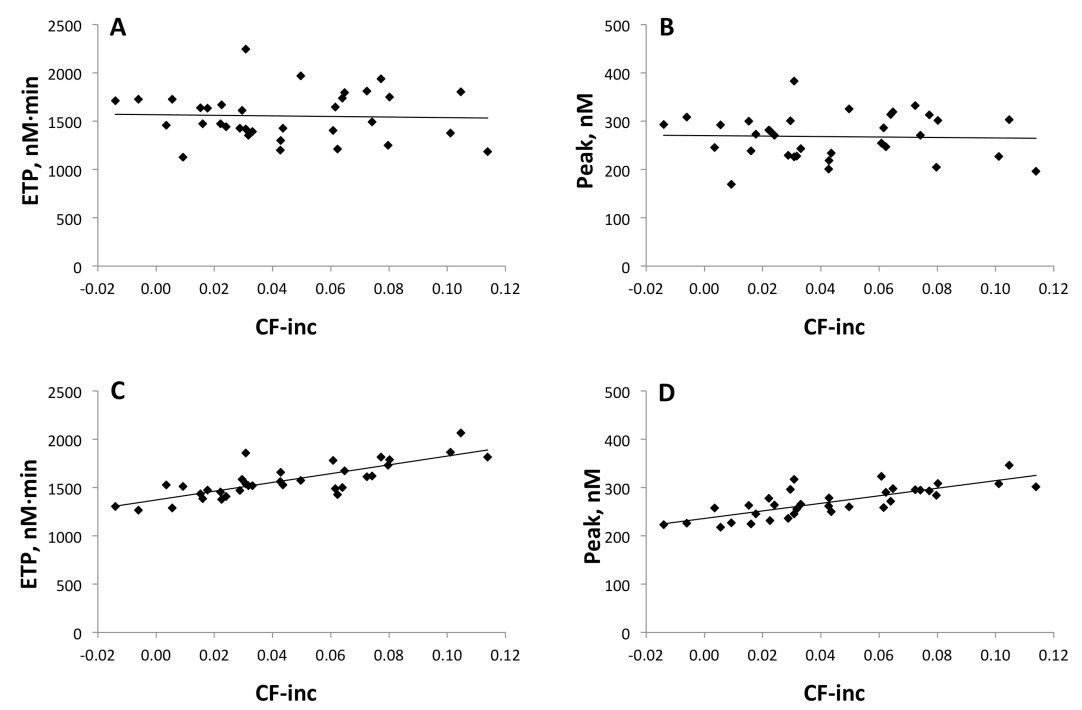

Figure 2. ETP and Peak versus temperature control estimation parameter CF-inc. $(A, B)-$ initial values; $(C, D)$ - values obtained after the elimination of calibrator activity errors.

Moreover, this relation allowed us to subtract a temperature dependent trend from the data and evaluate the variability of the data after this second correction.

Mean values, standard deviations and variation coefficients for corrected data are presented in table 2 . In total the two successive correction procedures both for temperature and for calibration reduced the variability of ETP and Peak by nearly $50 \%$.

Table 2. Mean values, standard deviations and variation coefficients for ETP and Peak, effect of temperature and calibration correction.

\begin{tabular}{|l|l|l|l|l|l|l|}
\hline & ETP & \multicolumn{3}{l|}{ Peak } \\
\cline { 2 - 8 } & initial & i.c. & i.c.+t.c. & initial & i.c. & i.c. + t.c. \\
\hline Mean & 1553.7 & 1571.0 & 1371.0 & 268.0 & 270.5 & 236.0 \\
\hline $\begin{array}{l}\text { Standard } \\
\text { deviation }\end{array}$ & 252.8 & 183.5 & 111.3 & 46.5 & 32.3 & 20.3 \\
\hline $\begin{array}{l}\text { Variation } \\
\text { coefficient, } \\
\%\end{array}$ & 16.3 & 11.7 & 8.1 & 17.4 & 11.9 & 8.6 \\
\hline
\end{tabular}

\section{Discussion}

\section{Normalization}


Normalization seems to be an effective way to reduce the inter-laboratory variation for the assay $y^{31,33,35,39}$. However, it does not seem capable to correct for all different parameters under all different conditions and the remaining variability is still relatively high $\sim 10 \%{ }^{39}$. Normalization could therefore only present a partial solution to the inter-laboratory variation for further clinical implementation, since its major drawback results in the persistence of the error in both the normal and test sample, which reduces both the sensitivity and specificity of the assay. Lastly, since normalization adds experimental conditions (as well as costs and time) to the assay by testing the reference plasma this may further increases the barrier for clinical implementation of TG.

\section{Calibration}

The calibration procedure is necessary to relate observed reaction velocities $(\mathrm{dF} / \mathrm{dt}$ ) to thrombin concentrations i.e. to quantify the amount of thrombin that is formed during an experiment. The classical approach to calibration, is to determine a CF once and use this value for all subsequent time points and experiments. In TG experiments this approach is not feasible, as during the experiment the inner filter effect and substrate consumption continuously increase the CF. Therefore, at every level of fluorescence another CF applies. In the experiments shown here the CF increases by $45 \%$ from the beginning to the end of the experiments. Moreover, the lack of stability of the excitation beam gives erroneous fluctuations of the signal. These could also lead to discrepancies between a predetermined CF and the measured TG signal. Lastly the colour of the plasma leads to a difference in the signal intensity since there are plasma constituents that absorb the energy of the emission wavelength which differ from sample to sample.

In the experimental procedure, the course of total amidolytic activity $f_{a}(t)$ is found, consisting of free thrombin $f_{g}(t)$ and thrombin bound to $\alpha 2 \mathrm{M}$. Because the velocity of formation of $\alpha 2 \mathrm{M}-\mathrm{T}$ in the pseudo first order reaction $\alpha 2 \mathrm{M}+\mathrm{T} \rightarrow \alpha 2 \mathrm{M}-\mathrm{T}$ is proportional to the concentration of free thrombin, with the reaction constant $k$ as the proportionality constant, the total amount of $\alpha 2 \mathrm{M}-\mathrm{T}$ formed is $k$ times the integral of the thrombin-time curve.

The contributions of $\mathrm{T}$ and $\alpha 2 \mathrm{M}-\mathrm{T}$ apart thus can be calculated by numerical solution of the differential equation $f_{a}(t)=f_{g}(t)+k \int_{0}^{t} f_{g}(t) d t$. In the end $(t \rightarrow \infty) \int_{0}^{\infty} f_{g}(t) d t=E T P$ and $k \int_{0}^{\infty} f_{g} d t=k \cdot$ ETP is the end level of $\alpha 2 \mathrm{M}-\mathrm{T}$.

In this procedure, the correct $\mathrm{k}$ is that one that allows the boundary condition that free thrombin becomes zero without ever having been significantly negative. In practice, this means that the point in time has to be estimated at which all free 
thrombin has disappeared. Various strategies can be employed that need not be discussed here; suffice it to say that the ideal solution has not yet been found. The essential point is that if $k$ is underestimated, part of the activity of $\alpha 2 \mathrm{M}-\mathrm{T}$ is attributed to free thrombin and contributes to the ETP, whereas when $k$ is overestimated part of the free thrombin is attributed to $\alpha 2 \mathrm{M}-\mathrm{T}$. Variations in the estimation of $\mathrm{K}$ might contribute to the residual error after compensation for temperature and calibration effects. Improvement in reliability of the estimation of $\mathrm{K}$ could be achieved by mathematical smoothing of the raw data prior to analysis.

Further reduction of the variability caused by calibration of the experiment can be sought in the internal calibration of the sample; for example, by utilizing the $\alpha 2 \mathrm{M}$ $T$ that is formed during the experiment. However, solutions that correct for the colour of the plasma and the inner filter effect have still to be found in order to be able to quantify the thrombin concentration in the sample in a reliable way.

\section{Temperature}

The variation that is introduced by temperature differences throughout the course of the experiment is at large an operator error. If the sample temperature is meticulously tuned to the temperature inside the fluorometer, and/or if the software in the fluorometer enforces the operator to pre-heat the sample long enough, the causes of the "hockey stick" disappear. A problem of uniformity of temperature within the instrument remains. We observed differences of up to $2^{\circ} \mathrm{C}$ in the same plate (manuscript in preparation). This suggests that for this type of experiments fluorometer should be equipped with more adequate temperature control. Currently samples are heated using air, which is typically known to be an insulator, if a more efficient conductor is used e.g. aluminium, the incubation time can be shortened to 5 min (manuscript in preparation). Which is more or less the same time that it takes to prepare the machine prior to the measurement, making the pre-heating step redundant.

\section{Recommendations}

Taking into account our current knowledge it stands to reason to perform a similar study $\mathrm{as}^{35}$ to determine how the suggested improvements to the CAT hold up in "real life" conditions. An improved inter-laboratory variability could expedite the clinical implementation of this versatile method. 


\section{References}

1. Macfarlane, R. G. \& Biggs, R. A Thrombin Generation Test. J. Clin. Pathol. 6, 3-8 (1953).

2. Pitney, W. R. \& Dacie, J. V. A Simple Method of Studying the Generation of Thrombin in Recalcified Plasma. J. Clin. Pathol. 6, 9-14 (1953).

3. Hemker, H. C., Wielders, S., Kessels, H. \& Béguin, S. Continuous registration of thrombin generation in plasma, its use for the determination of the thrombin potential. Thromb. Haemost. 70, 617-624 (1993).

4. Hemker, H. C. et al. Calibrated Automated Thrombin Generation Measurement in Clotting Plasma. Pathophysiol. Haemost. Thromb. 33, 4-15 (2003).

5. Dargaud, Y., Lienhart, A. \& Negrier, C. Prospective assessment of thrombin generation test for dose monitoring of bypassing therapy in hemophilia patients with inhibitors undergoing elective surgery. Blood 116, 5734-5737 (2010).

6. Millet, A. et al. Thrombin generation in patients with acquired haemophilia and clinical bleeding risk. Br. J. Haematol. 153, 136-139 (2011).

7. Bosch, Y. P. J. et al. Measurement of thrombin generation intra-operatively and its association with bleeding tendency after cardiac surgery. Thromb. Res. 133, 488-494 (2014).

8. Bloemen, S., Zwaveling, S., Ten Cate, H., Ten Cate-Hoek, A. \& de Laat, B. Prediction of bleeding risk in patients taking vitamin $\mathrm{K}$ antagonists using thrombin generation testing. Plos One 12, e0176967 (2017).

9. Tans, G. et al. Activated protein $\mathrm{C}$ resistance determined with a thrombin generation-based test predicts for venous thrombosis in men and women. Br. J. Haematol. 122, 465-470 (2003).

10. Hron, G., Kollars, M., Binder, B. R., Eichinger, S. \& Kyrle, P. A. Identification of patients at low risk for recurrent venous thromboembolism by measuring thrombin generation. JAMA 296, 397-402 (2006).

11. Buyue, Y., Whinna, H. C. \& Sheehan, J. P. The heparin-binding exosite of factor IXa is a critical regulator of plasma thrombin generation and venous thrombosis. Blood 112, 32343241 (2008).

12. Sanchez, C., Alessi, M. C., Saut, N., Aillaud, M. F. \& Morange, P. E. Relation between the antithrombin Cambridge II mutation, the risk of venous thrombosis, and the endogenous thrombin generation. J. Thromb. Haemost. JTH 6, 1975-1977 (2008).

13. Lavigne-Lissalde, G. et al. Prothrombin G20210A carriers the genetic mutation and a history of venous thrombosis contributes to thrombin generation independently of factor II plasma levels. J. Thromb. Haemost. JTH 8, 942-949 (2010).

14. Ay, C. et al. Prediction of venous thromboembolism in patients with cancer by measuring thrombin generation: results from the Vienna Cancer and Thrombosis Study. J. Clin. Oncol. Off. J. Am. Soc. Clin. Oncol. 29, 2099-2103 (2011).

15. Anžej Doma, S., Vučnik, M., Božič Mijovski, M., Peternel, P. \& Stegnar, M. Enhanced thrombin generation in women with a history of oral contraception-related venous thrombosis. Thromb. Res. 132, 621-626 (2013).

16. Hylckama Vlieg, A. et al. The risk of a first and a recurrent venous thrombosis associated with an elevated D-dimer level and an elevated thrombin potential: results of the THE-VTE study. J. Thromb. Haemost. 13, 1642-1652 (2015).

17. Martin-Fernandez, L. et al. Genetic Determinants of Thrombin Generation and Their Relation to Venous Thrombosis: Results from the GAIT-2 Project. PloS One 11, e0146922 (2016).

18. Maurer, E. et al. Targeting Platelet GPIb $\beta$ Reduces Platelet Adhesion, GPIb Signaling and Thrombin Generation and Prevents Arterial ThrombosisSignificance. Arterioscler. Thromb. Vasc. Biol. 33, 1221-1229 (2013). 
19. Ten Cate, H. \& Hemker, H. C. Thrombin Generation and Atherothrombosis: What Does the Evidence Indicate? J. Am. Heart Assoc. 5, (2016).

20. Tosi, F. et al. Increased plasma thrombin potential is associated with stable coronary artery disease: An angiographically-controlled study. Thromb. Res. 155, 16-22 (2017).

21. Nagashima, H. Studies on the different modes of action of the anticoagulant protease inhibitors DX-9065a and Argatroban. I. Effects on thrombin generation. J. Biol. Chem. 277, 50439-50444 (2002).

22. Walker, C. P. R. \& Royston, D. Thrombin generation and its inhibition: a review of the scientific basis and mechanism of action of anticoagulant therapies. Br. J. Anaesth. 88, 848863 (2002).

23. Brocal, I., Marco, P., Lucas, J., Verdú, J. \& Tarín, F. Thrombin generation test in patients under anticoagulant therapy with vitamin K antagonists. Thromb. Haemost. 101, 594-595 (2009).

24. Robert, S. et al. Is thrombin generation the new rapid, reliable and relevant pharmacological tool for the development of anticoagulant drugs? Pharmacol. Res. 59, 160-166 (2009).

25. Bloemen, S., Hemker, H. C. \& Al Dieri, R. Large inter-individual variation of the pharmacodynamic effect of anticoagulant drugs on thrombin generation. Haematologica 98, 549-554 (2013).

26. Chowdary, P. et al. Thrombin generation assay identifies individual variability in responses to low molecular weight heparin in pregnancy: implications for anticoagulant monitoring. Br. J. Haematol. 168, 719-727 (2015).

27. Tripodi, A. et al. How the direct oral anticoagulant apixaban affects thrombin generation parameters. Thromb. Res. 135, 1186-1190 (2015).

28. D’Alessio, A. et al. Long Term Low Molecular Weight Heparin Anticoagulant Therapy Modulates Thrombin Generation and D-dimer in Patients with Cancer and Venous Thromboembolism. Cancer Invest. 35, 490-499 (2017).

29. Reverter, J. C. et al. Inhibition of platelet-mediated, tissue factor-induced thrombin generation by the mouse/human chimeric 7E3 antibody. Potential implications for the effect of c7E3 Fab treatment on acute thrombosis and 'clinical restenosis'. J. Clin. Invest. 98, 863-874 (1996).

30. Keularts, I. M., Béguin, S., de Zwaan, C. \& Hemker, H. C. Treatment with a GPIIb/IIla antagonist inhibits thrombin generation in platelet rich plasma from patients. Thromb. Haemost. 80, 370-371 (1998).

31. Dargaud, $Y$. et al. Effect of standardization and normalization on imprecision of calibrated automated thrombography: an international multicentre study. Br. J. Haematol. 139, 303309 (2007).

32. Loeffen, R. et al. Preanalytic variables of thrombin generation: towards a standard procedure and validation of the method. J. Thromb. Haemost. JTH 10, 2544-2554 (2012).

33. Dargaud, Y. et al. Standardisation of thrombin generation test - which reference plasma for TGT?: An international multicentre study. Thromb. Res. 125, 353-356 (2010).

34. Dargaud, Y. et al. Proposal for standardized preanalytical and analytical conditions for measuring thrombin generation in hemophilia: communication from the SSC of the ISTH. J. Thromb. Haemost. 15, 1704-1707 (2017).

35. Perrin, J. et al. Large external quality assessment survey on thrombin generation with CAT: further evidence for the usefulness of normalisation with an external reference plasma. Thromb. Res. 136, 125-130 (2015).

36. Hemker, H. C., De Smedt, E. \& Hemker, P. W. During coagulation, thrombin generation shifts from chemical to diffusional control. J. Thromb. Haemost. 3, 2399-2400 (2005).

37. Hemker, H. C., Hemker, P. W., Al Dieri, R. \& others. The technique of measuring thrombin generation with fluorescent substrates: 4 . The H-transform, a mathematical procedure to 
obtain thrombin concentrations without external calibration. Thromb. Haemost. 101, 171177 (2009).

38. Hemker, H. C. \& Kremers, R. Data management in Thrombin Generation. Thromb. Res. 131, 3-11 (2013).

39. Dargaud, Y. et al. Evaluation of a standardized protocol for thrombin generation measurement using the calibrated automated thrombogram: An international multicentre study. Thromb. Res. 130, 929-934 (2012). 

Chapter 5

The Influence of Temperature on Thrombin Generation

Martijn Moorlag, H. Coenraad Hemker

Manuscript in preparation 


\section{Abstract}

Background Calibrated automated thrombography (CAT) is a global haemostasis assay and its outcome correlates well with bleeding and thrombosis risk. The assay itself has been proven to be highly sensitive for temperature, making temperature control of the experiment a necessary precondition for reproducible results. Temperature fluctuations throughout the experiment as well as between the wells in the plate have been shown to add to the variation in the results.

Objectives In this study we aim to identify and where possible quantify the influence of temperature on thrombin generation (TG). Moreover, by comparing the difference in response between defibrinated normal pooled plasma (NPP) and regular NPP we aim to further elucidate whether the control mechanisms of thrombin formation are diffusion limited.

Methods TG was measured at a range over temperatures from $30.5^{\circ} \mathrm{C}$ until $38.5^{\circ} \mathrm{C}$ with $2^{\circ} \mathrm{C}$ intervals in NPP and defibrinated NPP. The TG parameters where calculated using the new insights presented in chapter 6 . Prothrombin conversion and thrombin inactivation were assessed.

Results The TG parameters in NPP plasma where decreased at increasing temperature $-3.2 \% /{ }^{\circ} \mathrm{C}$ for the peak and $-4.4 \% /{ }^{\circ} \mathrm{C}$ for the ETP. Defibrinated plasma showed no temperature response for the peak and a $-1.4 \% /{ }^{\circ} \mathrm{C}$ decrease for the ETP. The calibration reaction increased with temperature by $3.8 \% /{ }^{\circ} \mathrm{C}$.

No anomalies have been observed as a result of the lower substrate concentration.

Conclusions TG parameters are decreased at increasing temperatures as a result of the difference in response of the pro- and anticoagulant processes of TG. Calibration of the TG increased the response to temperature changes in the TG parameters peak and ETP as a result of the opposite response to temperature between $T G$ and calibrator. 


\section{Introduction}

Coagulation assays, the Calibrated Automated Thrombogram (CAT) as described by Hemker ${ }^{1}$ being no exception, try to mimic the human physiological conditions as closely as possible. We all understand that concessions have to be made, because we cannot (as yet) represent the full physiology of the human body in in vitro coagulation tests. However, the one physiological aspect that most of the test really stringently adhere to is the representation of the human core body temperature, which is generally excepted to be around $37^{\circ} \mathrm{C}$ with a healthy state being considered to be between $36,1^{\circ} \mathrm{C}$ and $37,2^{\circ} \mathrm{C}^{2}$.

Coagulation is a highly complex mechanism that can easily be influenced by many factors one of these being temperature ${ }^{3,4}$. As a consequence, perfect thermostability is a condition sine qua non for reproducible measurement of thrombin generation (TG). It is therefore almost intuitively that we perform the test at $37^{\circ} \mathrm{C}$. Unfortunately, as is shown in chapter 4, temperature also happens to be one of the sources of error of the test results of TG and therewith one of limiting factors of comparison of results between laboratories.

The control of temperature in any device is often taken for granted and is generally considered to be the responsibility of the manufacturer. For the CAT it has been shown that both the instability of temperature throughout the 96 well plate ${ }^{5}$ as well as the temperature throughout the thrombin generation (TG) experiment ${ }^{3}$ influence the outcome of the TG experiment. It is not the purpose of this chapter to further elaborate on the technicalities of temperature control, but since they are important to understand, they are briefly presented in an appendix to this chapter, therewith accompanied by a brief description of an amelioration for the CAT to improve temperature stability and control.

TG is the result of prothrombin conversion and thrombin inactivation. If both processes would have approximately the same temperature coefficient, TG would be largely temperature independent and temperature control requirements would be easily met. Previously it has been shown, however, that lowering the temperature increases thrombin generation ${ }^{4}$. The assumption was that prothrombin conversion in the presence of fibrin is diffusion limited and therefore much less sensitive to lowering of temperature than thrombin inactivation ${ }^{4}$, which, like most (bio)chemical reactions will decrease its velocity (in a very rough approximation) by a factor two for every $10^{\circ} \mathrm{C}$ drop in temperature. Oddly enough, this observation is not supported by the clinic where hypothermia $\left(<32^{\circ} \mathrm{C}\right)$ in trauma patients, is associated with an inadvertent bleeding risk ${ }^{6}$. 
In the CAT method thrombin concentrations are calculated by comparing the fluorescence in the well where thrombin generates to that of the fluorescence that is generated in the calibrator well, where a known amount of $\alpha_{2}$ macroglobulinthrombin $\left(\alpha_{2} \mathrm{~m}-\mathrm{T}\right)$ complex converts the same substrate under the same experimental conditions (3). A difference in the temperature between the calibrator and TG well would therefore lead to either an over or underestimation of the thrombin concentration.

In order to investigate the extend of the influence of temperature disturbances on thrombin generation, we took a more fundamental approach by performing TG experiments at different temperatures. These experiments give us a good understanding of how the enzymatic reactions of the blood coagulation system respond to changes in temperature. And allow us to extrapolated and speculate on the influence of the more stochastic temperature fluctuations during the TG experiment.

\section{Material and methods}

TG was measured in normal pooled platelet poor plasma (PPP) at $5 \mathrm{pM}$ tissue factor (TF). A bespoke temperature controller was built into the fluorometer. It consists of a PID controller that steers a resistive heater attached to an aluminium mould. The aluminium decreases the overall temperature variation throughout the 96 wells plate to $<0.1^{\circ} \mathrm{C}$. The appendix to this chapter can be consulted for further information on the technical details behind the construction of the temperature controller.

\section{Fluorescence based measurement of thrombin activity}

The development of fluorescence intensity from 7-Amino-4-methylcoumarin (AMC) was measured in a 96-well plate fluorometer (Ascent reader, Thermolabsystems OY, Helsinki Finland) equipped with a 390/460 filter set (excitation/emission) and a dispenser. Immulon 2HB, flat-bottom 96-well plates (Dynex) are used. six readings are done per minute and experiments have been carried out in quadruplicate unless otherwise indicated. Per temperature two separate runs of experiments have been performed.

To each well, $80 \mu \mathrm{L}$ normal pooled plasma is added. Wells in which TG is measured receive $20 \mu \mathrm{L}$ of buffer I, containing 30 or $600 \mathrm{pM}$ of recombinant tissue factor (TF) and $24 \mu \mathrm{M}$ phospholipid preparation. Wells in which constant thrombin-like activity is measured receive $20 \mu \mathrm{L}$ of the $\alpha 2 \mathrm{M}$-thrombin $(\alpha 2 \mathrm{M}-\mathrm{T}$ ) solution which has a thrombin-like activity of $600 \mathrm{nM}$, resulting in a final concentration of $100 \mathrm{nM}$ thrombin like activity in the well. Thrombin generation (TG) was initiated by the addition of $20 \mu \mathrm{l}$ of ZGGR-AMC $(150 \mu \mathrm{M})$ and $\mathrm{CaCl}_{2}(16.6 \mathrm{mM})$. 
The dispenser of the fluorometer is flushed with mili q water, emptied and then flushed with a prewarmed solution of the substrate and calcium in buffer $I$ (referred to as FluCa) as stated in the Thrombinoscope manual. The "FluCa trigger" solution, a mixture of fluorogenic substrate and $\mathrm{CaCl}_{2}$ is prepared as follows. To $1782 \mu \mathrm{L}$ of buffer $\mathrm{B}$, at $37^{\circ} \mathrm{C}, 200 \mu \mathrm{L}$ of $1 \mathrm{M} \mathrm{CaCl}_{2}$ is added, then $18 \mu \mathrm{L}$ of a DMSO solution of $50 \mathrm{mM}$ ZGGR-AMC is squirted in and immediately vigorously mixed on a vortex. The pre-heating temperatures of the dispensing liquids have been adjusted to the measurement temperatures accordingly. At the start of the experiment, the instrument dispenses $20 \mu \mathrm{L}$ of the substrate/calcium mixture to all the wells, registers this as zero time, shakes them for $10 \mathrm{~s}$ and starts reading.

\section{Reagents}

Innovin recombinant human Tissue Factor (rTF) was used at final concentrations of $5 \mathrm{pM}$ and 100pM (Dade-Behring, Marburg, Germany). The calibrator $\left(\alpha_{2} \mathrm{~m}-\mathrm{T}\right)$ was prepared as previously described by Hemker et al. ${ }^{7}$. Procoagulant phospholipids (PL), containing 60\% dioleoyl PC, 20\% dioleoyl PS and 20\% dioleoyl PE, were prepared as described by ${ }^{8}$. ZGGR-AMC was purchased at Bachem (Basel, Switzerland) and dissolved in DMSO to a stock concentration of $50 \mathrm{mM}$.

\section{Buffers}

Buffer I used in the preparation of dilutions of the reagents: $20 \mathrm{mM}$ Hepes, 140 $\mathrm{mM} \mathrm{NaCl}, 0.02 \% \mathrm{NaN}_{3}$ and $5 \mathrm{mg} / \mathrm{mL} \mathrm{BSA}$ at $\mathrm{pH} 7.35$.

Buffer II Used for the solution of the fluorogenic substrate: $20 \mathrm{mM}$ Hepes, $0.02 \%$ $\mathrm{NaN}_{3}$ and $60 \mathrm{mg} / \mathrm{mL} \mathrm{BSA}$ at $\mathrm{pH}$ 7.35. The buffers are filtered using a corning filter system $250 \mathrm{~mL}$ with $0.2 \mu \mathrm{m}$ PES membrane and stored at $-20^{\circ} \mathrm{C}$.

\section{Plasma preparation}

Blood was obtained through antecubital venepuncture (1 volume trisodium citrate $0.13 \mathrm{M}$ to 9 volumes blood) in a $10 \mathrm{ml}$ vacutainer tube (BD, San Jose CA, USA) from apparently healthy volunteers after agreement to informed consent in concordance with the declaration of Helsinki. The blood was centrifuged twice at $2,821 \mathrm{~g}$ at room temperature for 10 minutes ( $\mathrm{min}$ ). Platelet-poor plasma was collected, and a pool (NP) was prepared and stored in $1 \mathrm{ml}$ containers at $-80^{\circ} \mathrm{C}$.

\section{Defibrination of normal pool plasma}

Plasma was defibrinated using $1 \mathrm{U} / \mathrm{ml}$ ancrod (NIBSC, South Mimms, UK). The dried ancrod powder was dissolved in milli q water so that $17 \mu \mathrm{l}$ could be added to 983 $\mu \mathrm{l}$ of citrated normal pool plasma to get the $1 \mathrm{U} / \mathrm{ml}$ final concentration. A clot was allowed to form during a $10 \mathrm{~min}$ incubation at $37^{\circ} \mathrm{C}$, and a subsequent $10 \mathrm{~min}$ incubation on ice. The fibrin clot was removed by winding it on a plastic spatula. 


\section{Data handling}

The raw data of optical density or fluorescence measurements of thrombin activity were exported to Excel (Microsoft Excel 2003) and corrected for substrate consumption and non-linearity of fluorescence with the concentration of the fluorophore by the method further described in chapter 6 of this book. The thrombin decay constants and the subsequent curves for prothrombin conversion and thrombin decay where determined according to the method of Kremers ${ }^{9}$.

The TG parameters used to represent the temperature effects are limited to the Calibrator level, the PEAK and the ETP. The Lagtime and Time to Peak are not included as they give an incomplete overview of the action of thrombin over time and are therefore considered to be less representative to elucidate the full scope of the influence of temperature on TG.

\section{Results}

\section{Temperature dependencies}

In the first column of tables 1 and 2 the temperature dependency of the calibration reaction. both in NPP (table 1) and in defibrinated NPP (table 2), are shown. The average increase in calibrator activity is around $4 \%$ per $1^{\circ} \mathrm{C}$, with little more increase in the calibrator from $36.5^{\circ} \mathrm{C}$ to $38.5^{\circ} \mathrm{C}$. This differs from the temperature dependencies of the TG reactions that are shown in columns 2-5 of tables 1 and 2, moreover there are also distinctive differences between the NPP and the defibrinated NPP experiments

The TG activity is represented here by the PEAK and the ETP values, in the NPP both values decrease with an increase of temperature, table 1 . columns 2 and 3 . The PEAK value decreases with an average of $3.2 \%$ per ${ }^{\circ} \mathrm{C}$ and the ETP with $4.4 \%$ per ${ }^{\circ} \mathrm{C}$. No stagnation of the decrease of Peak and ETP values is observed from 36.5 to $38.5^{\circ} \mathrm{C}$. The decrease of PEAK and ETP values is aggravated when the data are multiplied with their respective calibration factors, table 1 columns 4 and 5. This can be explained by the opposing temperature trends between the TG and the calibration experiments, this causes the lowest temperatures to be corrected less and the highest more. 
Table 1: Temperature dependence of the $T G$ experiment from $30.5^{\circ} \mathrm{C}$ until $38.5^{\circ} \mathrm{C}$ measured in NPP.

\begin{tabular}{|l|l|l|l|l|l|}
\hline \multirow{2}{*}{$\begin{array}{l}\text { Temp } \\
{ }^{\circ} \mathrm{C}\end{array}$} & \multirow{2}{*}{$\begin{array}{l}\text { Calibrator } \\
\text { slope }\end{array}$} & \multicolumn{3}{|l|}{$\mathrm{dF} / \mathrm{dt}$} & \multicolumn{2}{l|}{ Thrombin } \\
\cline { 3 - 6 } & $\begin{array}{l}\text { Peak Max } \\
\text { FU }\end{array}$ & $\begin{array}{l}\text { ETP } \\
\text { FU*min }\end{array}$ & $\begin{array}{l}\text { Peak } \\
\text { nmol/L }\end{array}$ & $\begin{array}{l}\text { ETP } \\
\text { nmol .min/L }\end{array}$ \\
\cline { 2 - 6 } & Average & Average & Average & Average & Average \\
\hline 30.5 & $145 \pm 1.9$ & $310 \pm 2.5$ & $1568 \pm 12.5$ & $224 \pm 1.8$ & $1140 \pm 9.1$ \\
\hline 32.5 & $163 \pm 6.4$ & $297 \pm 5.6$ & $1409 \pm 26.7$ & $187 \pm 3.5$ & $892 \pm 16.9$ \\
\hline 34.5 & $176 \pm 3.7$ & $277 \pm 3.4$ & $1301 \pm 16.1$ & $163 \pm 2.1$ & $772 \pm 9.5$ \\
\hline 36.5 & $185 \pm 2.6$ & $268 \pm 6.5$ & $1175 \pm 28.5$ & $145 \pm 3.5$ & $639 \pm 15.5$ \\
\hline 38.5 & $186 \pm 2.7$ & $225 \pm 4.4$ & $1039 \pm 20.2$ & $120 \pm 2.4$ & $552 \pm 10.7$ \\
\hline$\left(\% /{ }^{\circ} \mathrm{C}\right)$ & +3.8 & -3.2 & -4.4 & -4.6 & -5.6 \\
\hline
\end{tabular}

Table 2: Temperature dependence of the TG experiment from $30.5^{\circ} \mathrm{C}$ until $38.5^{\circ} \mathrm{C}$ measured in defibrinated NPP.

\begin{tabular}{|l|l|l|l|l|l|}
\hline \multirow{3}{*}{$\begin{array}{l}\text { Temp } \\
{ }^{\circ} \mathrm{C}\end{array}$} & Calibrator & \multicolumn{2}{|l|}{$\mathrm{dF} / \mathrm{dt} \mathrm{FU}$} & \multicolumn{2}{l|}{ Thrombin } \\
\cline { 2 - 6 } & Slope & $\begin{array}{l}\text { Peak Max } \\
\text { FU }\end{array}$ & $\begin{array}{l}\text { ETP } \\
\text { FU.min }\end{array}$ & $\begin{array}{l}\text { Peak } \\
\text { nmol/L }\end{array}$ & $\begin{array}{l}\text { ETP } \\
\text { nmol.min/L }\end{array}$ \\
\cline { 2 - 6 } & Average & Average & Average & Average & Average \\
\hline 30.5 & $154 \pm 2.3$ & $175 \pm 2.7$ & $730 \pm 11.1$ & $125 \pm 1.9$ & $589 \pm 8.9$ \\
\hline 32.5 & $167 \pm 7.1$ & $167 \pm 4.7$ & $683 \pm 19.3$ & $106 \pm 3.0$ & $495 \pm 14.0$ \\
\hline 34.5 & $190 \pm 3.1$ & $169 \pm 2.9$ & $680 \pm 11.5$ & $97 \pm 1.6$ & $448 \pm 7.6$ \\
\hline 36.5 & $200 \pm 2.1$ & $171 \pm 2.5$ & $678 \pm 9.9$ & $91 \pm 1.3$ & $399 \pm 5.8$ \\
\hline 38.5 & $205 \pm 1.9$ & $163 \pm 2.8$ & $652 \pm 11.3$ & $83 \pm 1.4$ & $337 \pm 5.8$ \\
\hline$\left(\% /{ }^{\circ} \mathrm{C}\right)$ & +3.8 & +0.001 & -1.4 & -2.8 & -4.5 \\
\hline
\end{tabular}

In defibrinated NPP the effect of a diminishing Peak and ETP is much less pronounced with no decrease of the Peak and a $1.4 \%$ per $1^{\circ} \mathrm{C}$ decrease of the ETP, table 2 columns 2 and 3 . Again this effect is amplified by the calibrator table 2 columns 4 and 5, where the multiplication with the calibration factor enhances the effect to 2.8 and $4.5 \%$ per $1^{\circ} \mathrm{C}$ decrease for the Peak and ETP respectively. The Lag-time in both instances, NPP and defibrinated NPP, decreases with increasing temperature from $1.16 \mathrm{~min}$ at $30.5^{\circ} \mathrm{C}$ to $0.83 \mathrm{~min}$ at $38.5^{\circ} \mathrm{C}$, suggesting a more procoagulant state at higher temperatures. 
An approximation of the decay constants for NPP and defibrinated NPP can be found in table 3 an increase in the constants can be observed with increasing temperature.

Table 3. Approximated decay constants for TG experiments at a range of temperatures

\begin{tabular}{|l|l|l|}
\hline Temperature & $\begin{array}{l}\text { Decay constant defibrinated } \\
\text { NPP }\end{array}$ & $\begin{array}{l}\text { Decay constant } \\
\text { NPP }\end{array}$ \\
\hline 30.5 & -0.71 & -0.42 \\
\hline 32.5 & -0.85 & -0.49 \\
\hline 34.5 & -0.90 & -0.56 \\
\hline 36.5 & -1.04 & -0.63 \\
\hline 38.5 & -1.04 & -0.72 \\
\hline
\end{tabular}

Figure 1 illustrates the prothrombin conversion curves that have been obtained using the method by Kremers et al. ${ }^{9}$ and the decay constants from table 3. It shows an overall slight increase in prothrombin conversion with time. In defibrinated NPP, figure 2, the increase in prothrombin conversion is more pronounced.

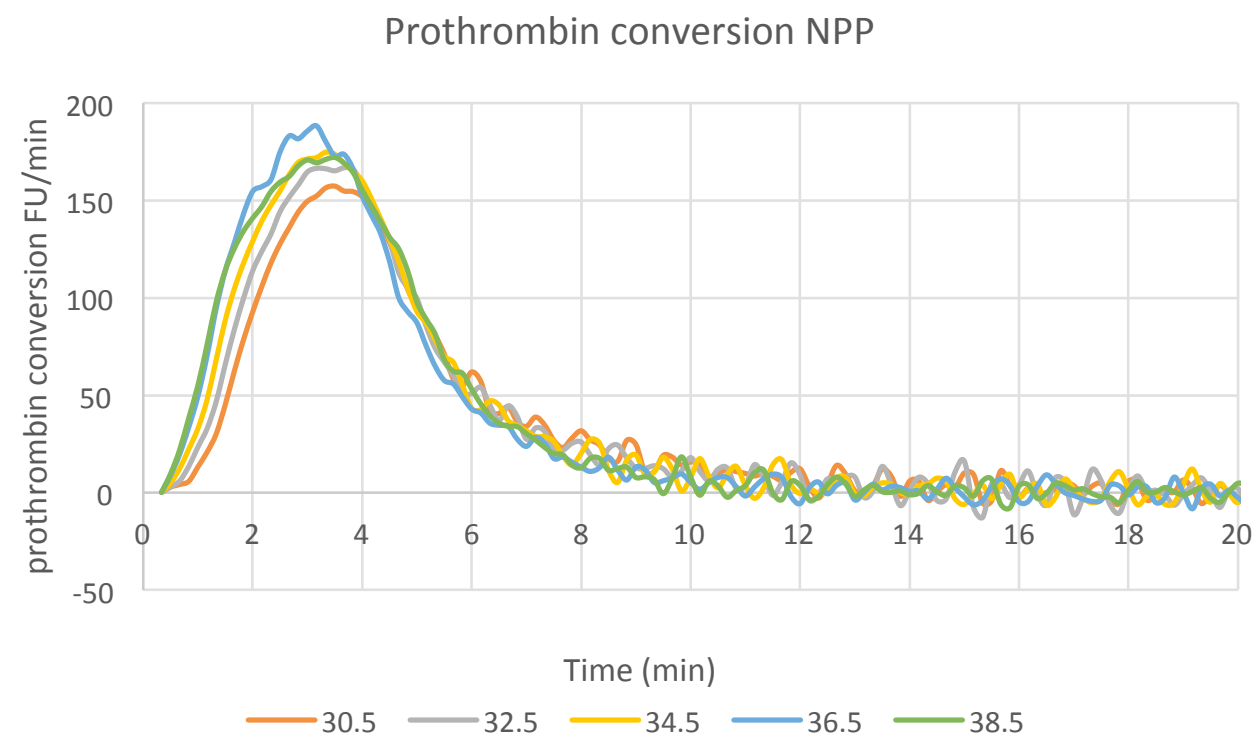

Figure 1: approximation of prothrombin conversion in NPP measured at $5 p M$ TF. 


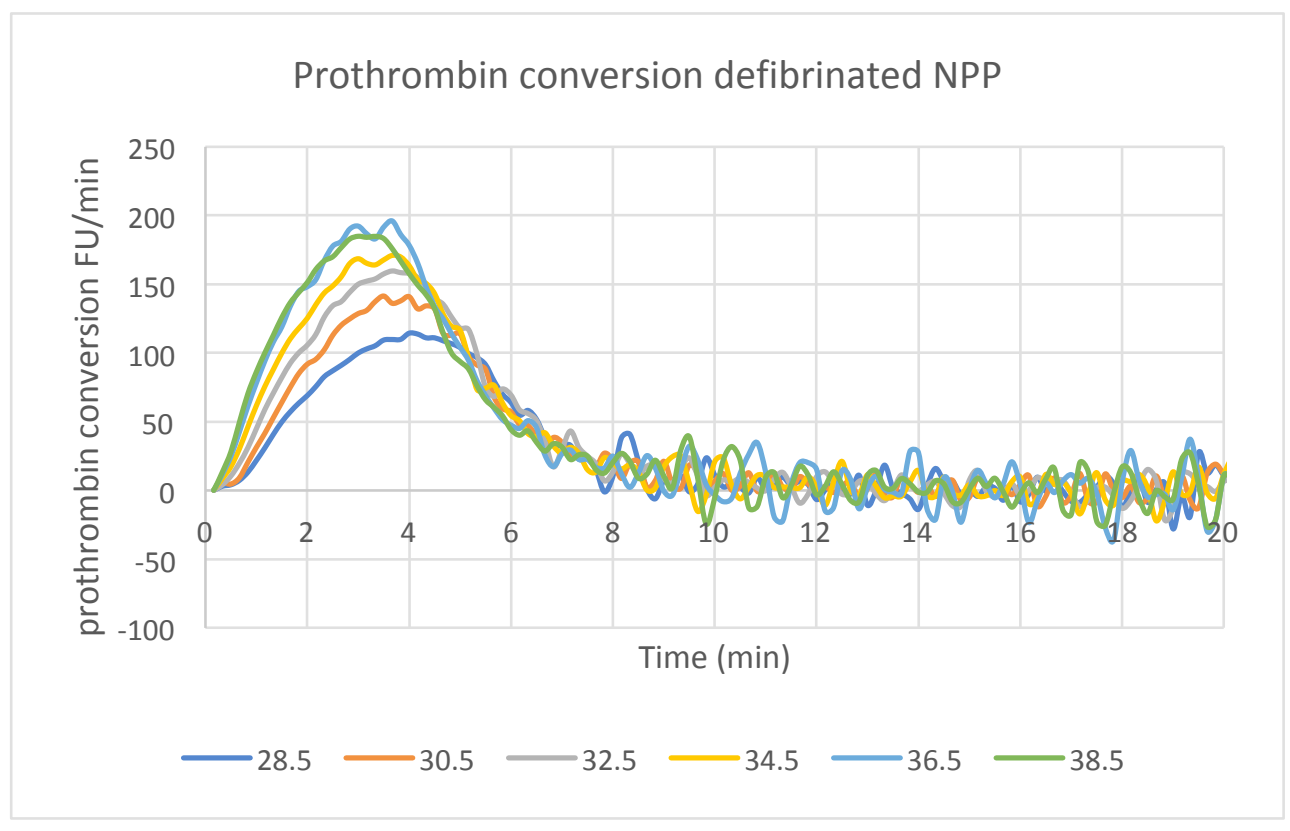

Figure 2: approximation of prothrombin conversion in NPP measured at 5pM TF.

\section{Substrate concentration}

The maximum level of fluorescence that was attained in these experiments with a substrate concentration of $150 \mu \mathrm{M}$ was $3200 \mathrm{FU}$. The highest level of fluorescence over the functional part of the TG curve was measured at $2000 \mathrm{FU}$ for the $100 \mathrm{pM}$ TF concentration at $28.5^{\circ} \mathrm{C}$.

\section{Discussion}

Humans are homeothermic (warm blooded) species, they generally have a core body temperature of $37^{\circ} \mathrm{C}$. Several mechanisms of heating and cooling are applied to sustain this steady state thermal equilibrium. These mechanisms combined with the irregular geometry of the human body, lead to a complicated threedimensional temperature distribution. This causes a temperature gradient that is not just longitudinal but also axial. Moreover, this gradient is greatly influenced by the outside temperature. To assume that $37^{\circ} \mathrm{C}$ is therefore the most representative temperature to measure coagulation is by itself up for debate.

Heat loss is, in part, regulated through conduction in the peripheral blood flow. The large vessels that lie deeper in the extremities are in parallel with one another. 
This allows the warmer outward flowing arterial blood to give off heat to the inward flowing venous blood. This so-called counter-current principle causes a steeper axial temperature gradient. When thrombosis, that is often found in veins of the lower legs, is considered, it just as likely as not that it occurs under lower temperatures $<35^{\circ} \mathrm{C}$ then at $37^{\circ} \mathrm{C}$. And if the temperature was lower, a different dynamic of the coagulation system led to its formation.

Moving beyond the temperature differences it can be further narrowed down to the site of an injury where a temperature gradient can very well be expected between the underlying tissue and the ruptured skin and vessels that are exposed to the air. Heat loss of the blood and tissue as they are exposed to air is very likely to be expected at the site where the plug has to be formed to halt bleeding.

Our results show that thrombin generation increases considerably when the temperature drops. In the $30.5-38.5^{\circ} \mathrm{C}$ interval the ETP increases by nearly $5.6 \%$ per degree and the peak by $4.6 \%$. Thereby providing a possibly explanation for the degree of variation that can be a result of a temperature gradient within the plate and/or throughout the experiment.

The increase of thrombin generation with decreasing temperature is much more apparent in the presence of a fibrin web, this contradicts the previously made assumption that in the presence of a fibrin web prothrombin conversion is diffusion limited and therefore less temperature dependent than thrombin inactivation, which is a bi-molecular reaction in free solution ${ }^{4}$. However, the prothrombin conversion curves and decay constants illustrate that both prothrombin conversion and thrombin decay increase with increasing temperature. The overall result of a decrease in TG with increasing temperature can therefore only be explained by the fact that the procoagulant reactions are influenced less by temperature than the decay reactions. This again hints to the possibility of diffusion limited reaction rates in thrombin formation but these results are however not conclusive enough to either confirm or reject the previously posed hypothesis in the work by Hemker et al. ${ }^{4}$.

The contradictory results of the TG with lowering temperatures are a good example of the surplus value of measuring beyond the formation of a clot. Whilst clot formation i.e. the clotting time are delayed with decreasing temperature, overall TG as represented by the PEAK and the ETP are increased, indicating a more prothrombotic phenotype. Interestingly it is believed that bleeding is a result of hypothermia in trauma patients, the idea that this bleeding is amongst other things a result of delayed reaction rates of the clotting enzymes is supported by clotting times ${ }^{6}$ but now contradicted by TG results. The result of the differences between these reactions in NPP is that the overall lower amount of thrombin is 
able to cleave more substrate over a longer time at lower temperatures where it is cleared less rapidly relative to its formation.

The differences in the influence of temperature between the TG- and the calibrator well and deviations from the standard $\left(37^{\circ} \mathrm{C}\right)$ temperature will influence the results of a CAT experiment. A multiplication by a calibration standard that was also under the influence of the same temperature fluctuation can therefore aggravate the variation that is observed in either of the two parameters alone.

Being the first series of experiments to be performed with a $150 \mu \mathrm{M}$ substrate concentration, albeit out of necessity, no adverse consequences where observed throughout the experiment. Moreover, the maximum fluorescence of the functional part of the TG curve never surpassed more than $60 \%$ of the maximum fluorescence attainable for this substrate concentration in any of the experiments. The defibrinated samples do reach the maximum fluorescence over the course of 60 min that the experiment is run. However, this is due to the large contribution of $\alpha 2 \mathrm{M}-\mathrm{T}$, that clears a larger portion of thrombin in the absence of fibrin, the relevant part of the TG curve has finished long before this value is reached. Therefore, the most important precondition for the calculation method that was used and that will be further elaborated upon in chapter 6 still seems to be fulfilled. 


\section{References}

1. Hemker, H. C. et al. Calibrated Automated Thrombin Generation Measurement in Clotting Plasma. Pathophysiol. Haemost. Thromb. 33, 4-15 (2003).

2. SF Morrison. Regulation of body temperature. in Medical physiology chap 59 (Elsevier).

3. De Smedt, E. \& Hemker, H. C. Thrombin generation is extremely sensitive to preheating conditions. J. Thromb. Haemost. 9, 233-234 (2011).

4. Hemker, H. C., De Smedt, E. \& Hemker, P. W. During coagulation, thrombin generation shifts from chemical to diffusional control. J. Thromb. Haemost. 3, 2399-2400 (2005).

5. Liang, Y., Woodle, S. A., Shibeko, A. M., Lee, T. K. \& Ovanesov, M. V. Correction of microplate location effects improves performance of the thrombin generation test. Thromb. J. 11, 12 (2013).

6. Wolberg, A. S., Meng, Z. H., Monroe III, D. M. \& Hoffman, M. A systematic evaluation of the effect of temperature on coagulation enzyme activity and platelet function. J. Trauma Acute Care Surg. 56, 1221-1228 (2004).

7. Hemker, H. et al. The calibrated automated thrombogram (CAT): a universal routine test for hyper-and hypocoagulability. Pathophysiol. Haemost. Thromb. 32, 249253 (2002).

8. Rosing, J., Tans, G., Govers-Riemslag, J., Zwaal, R. \& Hemker, H. C. The role of phospholipids and factor $V a$ in the prothrombinase complex. J. Biol. Chem. 255, 274-283 (1980).

9. Kremers, R. M. W., Wagenvoord, R. J. \& Hemker, H. C. The effect of fibrin(ogen) on thrombin generation and decay. Thromb. Haemost. 112, 486-494 (2014). 


Chapter 6

The relation between thrombin concentration and reaction velocity as measured from the conversion of fluorogenic substrate.

Martijn Moorlag, Saartje Bloemen, Konstantin Guria, Piet W. Hemker, Nikolaus Binder, Bas de Laat, H. Coenraad Hemker. Manuscript in preparation 


\section{Abstract}

Background The course of the formation and decay of thrombin in clotting plasma can be measured from the conversion of a thrombin-substrate rendering a fluorescent product. The intensity of the measured fluorescence contains all information from which the exact course of thrombin concentration can be obtained. However, presently, there is no ideal solution for the calculation of a thrombin generation curve from the time course of fluorescence intensity. The two main factors complicating the calculation of the ideal fluorescence are the inner filter effect and substrate consumption.

Methods calibrated automated thrombogram TG experiments where performed in normal pooled plasma, icteric plasma and a range of mixtures of the normal and the icteric plasma. Optical density measurements of these plasma samples and the mixtures where performed in a spectrophotometer.

Results When the fluorescence intensity at $300 \mu \mathrm{M}$ AMC is normalized to 1000 $\mathrm{AU}$ and 2090 is used as the value of $F_{\text {max }}$ the formula $F_{\text {ideal }}=-F_{\text {max }} \ln \left(1-F_{\text {exp }} /\right.$ $\left.F_{\text {max }}\right)$ can be used to eliminate the inner filter effect for any fluorometer of the type used for the experiments. After such correction the decrease of reaction velocity appeared to follow Michaelis-Menten kinetics.

The colour of the plasma causes decrease of fluorescence intensity proportional to the optical density at the excitation and emission wavelengths.

Conclusions The IFE can be eliminated with the formula: $F_{\text {ideal }}=-F_{\text {max }} \ln \left(1-F_{\text {exp }} / F_{\text {max }}\right)$ in any fluorometer of the type used after normalization of the fluorescence intensity at $300 \mu \mathrm{M} \mathrm{AMC}$ to $1000 \mathrm{AU}$ and using 2090 as the value of $F_{\max }$. The conversion of substrate by $\alpha_{2} \mathrm{M}-\mathrm{T}$ can be described by the classical Michaelis-Menten parabola $\frac{d P}{d t}=E \cdot k_{\text {cat }} . \mathrm{S} /(\mathrm{Km}+\mathrm{S})$ Where $\mathrm{V}_{\max }$ is the maximal velocity at infinite substrate concentration and $\mathrm{Km}$ the concentration at

which $\frac{d P}{d t}=\frac{1}{2} V_{\text {max }}$. 


\section{Introduction}

The course of the formation and decay of thrombin in clotting plasma can be measured from the conversion of a thrombin-substrate rendering a fluorescent product. The intensity of the measured fluorescence contains all information from which the exact course of thrombin concentration can be obtained. However, presently, there is no ideal solution for the calculation of a thrombin generation curve from the time course of fluorescence intensity. This is caused by the fact that the calibration factor that converts the observed reaction velocity into an enzyme concentration changes during the reaction.

If it were to be stable, the rate of fluorescence increase would be linearly proportional to the thrombin concentration and a simple first derivative of the fluorescence trace would represent the thrombin generation curve. Moreover, in this ideal scenario one calibration factor, relating a known thrombin concentration to the reaction velocity would suffice to obtain a TG-curve that represents thrombin concentration from the trace. However, in real life, the measured reaction-velocity is not proportional to the concentration, as is easily seen from the fluorescence trace that is observed when a fixed amount of thrombin activity is added to the reaction mixture, i.e. in a TG calibrator experiment.

Two mechanisms are thought to be responsible for this phenomenon: The inner filter effect (IFE) which causes the fluorescence intensity to decrease proportionally to the increasing concentration of fluorescent product. Secondly, substrate consumption (SC), which causes the reaction velocity to decrease proportionally with enzyme concentration as the reaction proceeds.

Moreover, the same amount of fluorescent product will give a lower signal in heavily coloured (e.g. icteric plasmas) than in lighter ones, therefore a reaction velocity appears to be lower in darker plasmas. This does not change the proportionality between observed reaction velocity and thrombin concentration in the course of the experiment but it does thwart comparison between plasmas, notably with a normal reference plasma.

Although it is certain that the abovementioned phenomena will have the effects discussed, it has never been proven that the curvature of the fluorescence trace observed in a calibrator-experiment is indeed explained by them. Neither is it known what the contribution is of IFE and SC to the observed curvature, nor whether in plasma they obey the relations that theory predicts. Notably, it is not known whether the enzymatic conversion of the fluorescent product can be described by the Michaelis-Menten relation or that more complicated enzyme kinetics apply. 
In the following chapter we have tried to analyse the causes of the non-linearity between enzyme concentration and observed reaction velocity in the course of the reaction. Knowledge of this relation is of practical interest. Present day attempts to extract a thrombin generation curve from a fluorescence trace are based on the parallel measurement of calibration experiment in which a known fixed amount of thrombin activity represented by the $\alpha 2$ macroglobulin-thrombin-complex $(\alpha 2 \mathrm{M}$ T), is compared to the "staple experiment" in which thrombin is generated ${ }^{1}$. An algorithm than compares the fluorescence curve from the staple well to that in the calibrator well and corrects for IFE and SC. Because the two experiments are carried out in the same plasma the colour of the plasma does not influence the results. Such algorithms work by fitting a mathematical function to the calibrator trace and from that function calculate the appropriate calibration factor at every level of fluorescence (see further ${ }^{2}$ ). Such comparisons are not based upon insight in the mechanisms (IFE, SC and plasma colour) that cause the distortion of the signal and they can be an important source of error in practice (see chapter 4 of this book). It is our aim to provide a calculation method that is based on insight in the underlying phenomena responsible for the non-linear relationship between observed reaction rate and thrombin-concentration curvature, which allows for reconnaissance of the sources of error and hence their elimination.

\section{Materials \& Methods}

\section{Chemicals/buffers}

Fluorogenic substrate, Z-Gly-Gly-Arg-7-amino-4-methylcoumarin (ZGGR-AMC), was obtained from Bachem (Bubendorf, Switzerland). AMC, the fluorogenic product part of the substrate, was obtained from Sigma Aldrich (Saint Louis, Missouri, USA). The a2Macroglobulin-Thrombin ( $\alpha 2 \mathrm{M}-\mathrm{T}$ ) complex that is used as a calibrator was prepared in house as previously described ${ }^{1}$. Two different Hepes buffers where prepared both contained: Hepes $20 \mathrm{mM}, \mathrm{pH} 7.35,140 \mathrm{mM} \mathrm{NaCl}, 0.02 \% \mathrm{NaN}_{3}$ to the $1^{\text {st: }}: 60 \mathrm{~g} / \mathrm{l}$ bovine serum albumin (BSA) (Sigma Aldrich, A-7030) was added and to the $2^{\text {nd }}: 5 \mathrm{~g} / \mathrm{BSA}$.

\section{Plasma preparation}

Blood was obtained through antecubital venipuncture ( 1 volume trisodium citrate $0.13 \mathrm{M}$ to 9 volumes blood) from healthy individuals who consented to participate in this study. BD vacutainer tubes were used for the collection of the blood samples (BD vacutainer system, Roborough, Plymouth, UK). Platelet poor plasma was prepared by centrifuging twice at 2,900 $\mathrm{g}$ for $10 \mathrm{~min}$ at room temperature, PPP was preferably prepared within $2 \mathrm{~h}$ after venipuncture. 


\section{Fluorometer/spectrophotometer}

Fluorescence measurements were performed either in a 96-well plate fluorometer (Ascent reader, Thermolabsystems OY, Helsinki Finland) equipped with a 390/460 filter set (excitation/emission) and a dispenser using Immulon 2HB, flat-bottom 96well plates (Dynex) or in a Ceveron Alpha device (Technoclone, Vienna, Austria). All optical density (OD) measurements were performed in plastic disposable cuvettes in a Shimadzu UV-1800 (Shimadzu, Kyoto, Japan). Measurements were calibrated on a "blank" demineralised water sample.

\section{Correcting for the inner filter effect}

When a series of AMC-concentrations is measured in a fluorometer, the increase of fluorescence is not linear with the AMC concentration, due to the inner filter effect (IFE), i.e. the absorption of light by fluorophore already present. The relation between the measured fluorescence $\left(F_{\text {exp }}\right)$ and the concentration of the fluorophore $(C)$ is known to be:

$\mathrm{F}=A C e^{-b C}$

with $\mathrm{F}=$ fluorescence, $\mathrm{C}=$ concentration and $\mathrm{A}$ and $\mathrm{b}$ constants $^{3}$ the derivation of this formula can be found in the supplementary materials to this chapter.

This formula (1) predicts a maximum in the fluorescence-concentration relation that in the practice of a thrombin generation measurement is never reached. In practice, the higher $30 \%$ of the fluorescence range is to be avoided, because the first derivatives that are calculated from the differences between high fluorescence values are beset with noise making them erratic and hence reaction velocities cannot be reliably calculated. Therefore, we can accept an approximation that proves its suitability within the first $2 / 3$ of the fluorescence range. In the standard setup of CAT experiments $417 \mu \mathrm{M}$ of substrate is used of which about $60 \%$ is converted (see figure 1). As can be appreciated from figure 1 , over the range of the lower $2 / 3$ of fluorescence intensities formula (1) can be conveniently replaced by:

$$
F_{\text {exp }}=F_{\max }\left(1-e^{-k C}\right)
$$




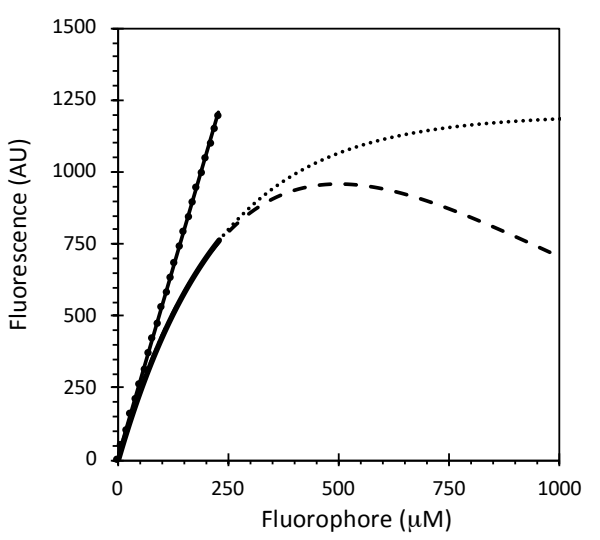

Figure 1: The relation between concentration of fluorophore and fluorescence.

Dashed line: Relation according to formula (1). Dotted line: Relation according to formula (2). Drawn line: Interval over which the discrepancy between the two formulas was $<0.5 \%$. Straight line: data after application of the correction formula.

We verified the extent to which our experimental data obey this theory. We determined the fluorescence values over the range of 0-300 $\mu \mathrm{M}$ fluorophore in five different fluorometers (see experimental section) and fitted both formula (1) and formula (2) to these points. We then calculated the theoretical fluorescence values and compared them among each other and with the experimental values (figure 2). No systematic deviation from identity was found.
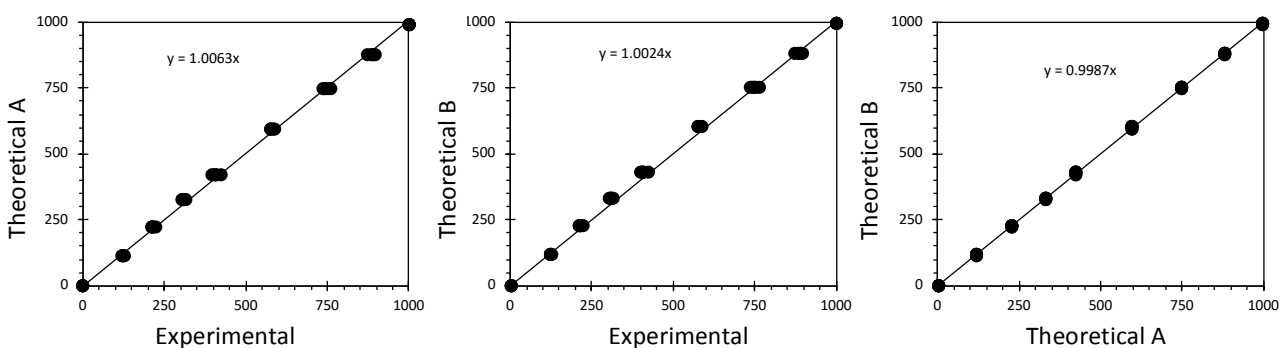

Figure 2. The relation between experimental and calculated fluorescence.

The left frame: fit of experimental points to formula (1). $A=4.9 ; B=0.0013$. Middle frame: fit of experimental points to formula (2). $F_{\max }=1790 ; k=0.0027$. Right frame: Comparison of the calculated values.

In practice we want to eliminate the IFE by calculating $F_{\text {ideal }}$ i.e. the fluorescence value that is linearly proportional to the concentration of fluorophore: $F_{\text {ideal }}=$ $H . C$; where $\mathrm{H}$ is the calibration constant that converts arbitrary fluorescence units in $\mu \mathrm{M}$ fluorophore. This requires $\mathrm{C}$ to be eliminated from the set of equations: 


$$
F_{\text {exp }}=A C e^{-b C} \text { and } F_{\text {ideal }}=H . C
$$

This calculation involves the ProductLog function (also known as the Lambert W or omega function) it cannot be expressed in elementary functions and is not available in the usual spreadsheet and calculation programs ${ }^{4}$. When we use formula (2), however elimination of $\mathrm{C}$ from the equations $F_{\text {exp }}=F_{\max }\left(1-e^{-k C}\right)$ and $F_{\text {ideal }}=$ H.C is readily possible and yields:

$F_{\text {ideal }}=F_{\text {max }} \ln \left(1-F_{\text {exp }} / F_{\text {max }}\right)$

The straight line with dots in figure 1 represents the values of $F_{\text {ideal }}$ resulting from the application of this formula to the data obtained with formula (1) (line) and (2) (dots). In figure 1, the dashed line represents fluorescence as a function of the fluorophore concentration as found from formula (1), with $A=5$ and $b=0.002$. The dotted curve in figure 2 is overlaid with a drawn line in the lower $2 / 3$ of fluorescence values. The dashed line is the result of the application of formula (3) to the dotted line. The straight line reflects the ideal proportionality of fluorescence and concentration of fluorophore. It is seen that for fluorescence values up to $67 \%$ of the theoretical maximum the application of formula (3) approaches this ideal proportionally. The error that is introduced by our approximation of the Lambert- $W$ formula remains $<1 \%$ in the useful part of the curve. Again, we stress that in practice, it can easily be avoided that fluorescence attains levels in which our approximation does no longer hold. Even at maximal substrate concentration $(417 \mu \mathrm{M})$ the amount of product formed during a normal thrombin generation experiment does not attain that level unless the experiment is prolonged far beyond the usual time range. Later (Chapter 8) we will show that it is preferable to use significantly lower substrate concentrations in which case fluorescence intensities remain far from the critical zone.

\section{Results}

We measured the fluorescence of a concentration series of AMC in pooled normal plasma in five different Ascent fluorometers (in fourfold) and found that, apart from the value of $A$, unique for every instrument, they all responded in the same way. If the fluorescence units found for a fixed concentration of fluorophore (e.g. $300 \mu \mathrm{M}$ ) is normalised to a fixed value (e.g. $1000 \mathrm{AU}$ ) than the experimental curves superimpose (figure 3). The experimental points fitted to the equation $F_{\text {exp }}=$ $3.6 C e^{-0,0009 C}$ as well as to the formula $F_{\text {exp }}=1790\left(1-e^{-0,0027 C}\right)$. From the latter we deduced the correction formula $F_{\text {ideal }}=-1900 \ln \left(1-F_{\text {exp }} / 1900\right)$ to convert the experimental fluorescence values into values that were proportional to the concentration, so that $C=-(1900 / 3.6) \ln \left(1-F_{\text {exp }} / 1900\right)$ (figure 3$)$. 


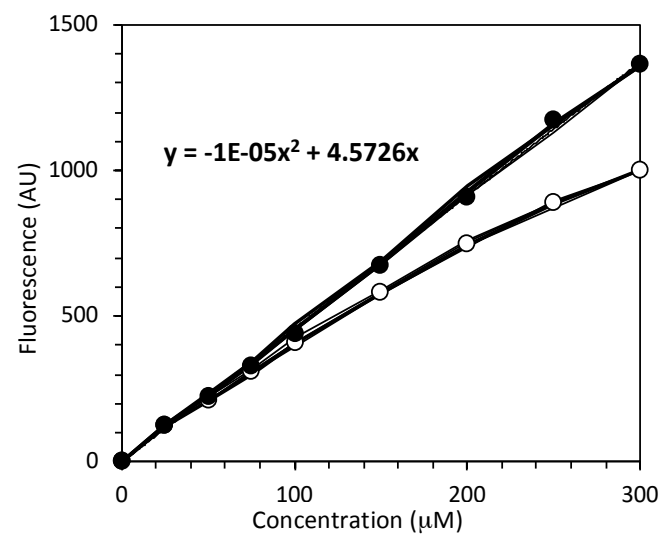

Figure 3. The relation between fluorescence and the concentration of fluorophore.

Data from five fluorometers normalized to $1000 \mathrm{AU}$ units of fluorescence at $300 \mu \mathrm{M}$ of fluorophore. O Average of individual experimental data. Average points after correction with formula (3). Drawn lines join individual data both before and after correction. $F_{\max }$ is found by minimizing the quadratic term in a fitted parabolic trendline.

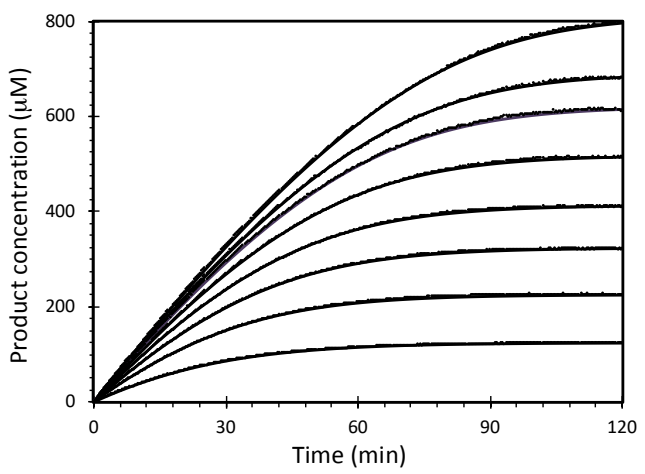

Figure 4. Development of product by $100 \mathrm{nM}$ thrombin at a series of concentrations of ZGGR-AMC. From top to bottom: $800-100 \mu \mathrm{M}$ ZGGR-AMC at $100 \mu \mathrm{M}$ intervals. Dots: Experimental points, lines fitted progress curve assuming Michaelis-Menten kinetics.

In a Ceveron fluorometer, instead of adding fluorophore, we ran calibration experiments with $100 \mathrm{nM}$ of $\alpha_{2} \mathrm{M}-\mathrm{T}$ at a series of concentrations of ZGGR-AMC until the substrate was completely consumed. Consequently, the end levels represent the fluorescence resulting from the fluorophore at the original substrate concentrations (figure 5). 


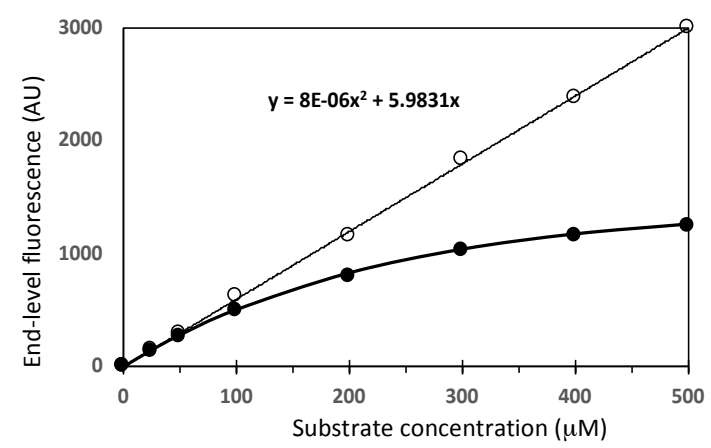

Figure 5. The inner filter effect and its correction

- End-level fluorescence. $\bigcirc$ Corrected points. Thick drawn line: Theoretical Fit. Thin drawn line: Linear regression line. Two times SD falls within the diameter of the points.

The theoretical curve that fitted the experimental points was $F_{\text {exp }}=5.8 C e^{-0.001695 C}$ and the, undistinguishable approximative curve was $F_{\text {exp }}=1420\left(1-e^{-0.0044 C}\right)$ consequently, the idealised values can be found as $F_{\text {ideal }}=1420 * \operatorname{Ln}\left(1-\frac{F_{\text {exp }}}{1400}\right)$ and $C=F_{\text {ideal }} / 6.187$. Using these values, the curves in figure 6 were obtained.

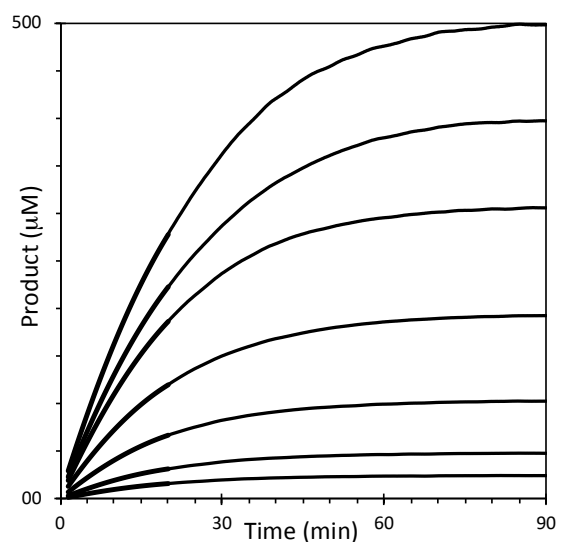

Figure 6. Formation of product in a Ceveron fluorometer.

A series of concentrations of ZGGR-AMC are tested. From top to bottom: 500, 400, 300 200,100, 50, $25 \mu \mathrm{M}$. Bold: Quadratic fit to the curve

\section{Substrate consumption}

The kinetic constants of the enzyme-substrate interaction can be derived from the curves of figure 6 . Firstly, the initial velocities were obtained from a parabolic fit to the first $2 / 3$ of the product range (Chapter 7 ). When these are plotted against the concentration, a hyperbola is found with $\mathrm{Km}=555 \mu \mathrm{M}$ and $\mathrm{V}_{\max }=22$ and a $\mathrm{k}_{\text {cat }}$ of $0.22 \mathrm{~min}^{-1}$ (figure 7). From the Lineweaver-Burk plot it becomes clear that the 
intercepts with the axes are so near to the origin, that only the slope, i.e. $\mathrm{Km} / \mathrm{k}_{\text {cat }}=25.2 \mu \mathrm{M} / \mathrm{min}$ can be obtained with some precision. A more precise measurement would require measurements at higher substrate concentrations
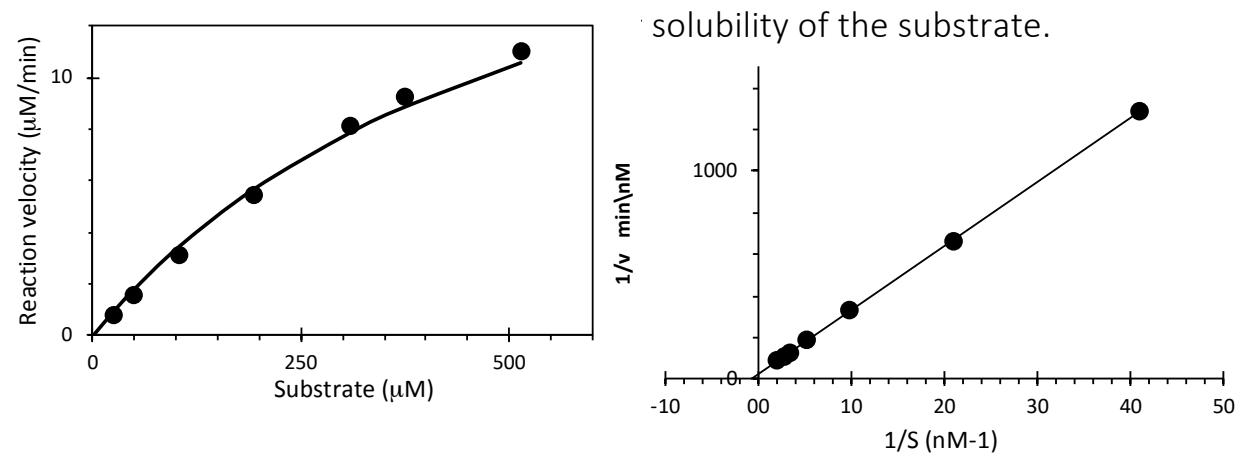

Figure 7. Initial velocity as a function of substrate concentration.

Left frame: Dots: Experimental points; Line: fitted hyperbola. Right frame: LineweaverBurke plot of the same data

From the course of product in time (figure 6), the substrate concentration can be deduced (i.e. the initial concentration - product) as well as the reaction velocity (i.e. the slope of the curve) at any point in time. Such concentration-velocity pairs can be plotted in a graph similar to the left frame of figure 7 . The reaction velocities have been calculated over one-minute intervals, subjecting them more to experimental noise than the initial velocities. Figure 8 represents the results obtained from the ensemble of curves shown in figure 6 . The broad grey line is the hyperbola on basis of the initial velocities. We conclude that the system obeys Michaelis-Menten kinetics over the whole range of the experiment.

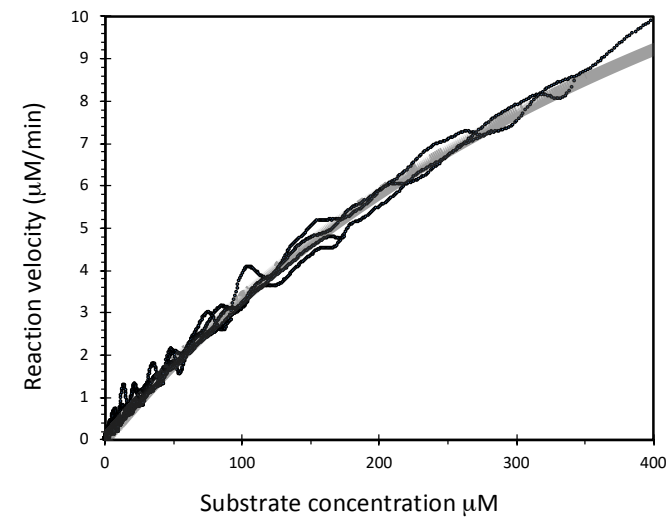

Figure 8. Concentration-reaction velocity plot derived from data in figure 6.

Black points: concentration-velocity pairs from the seven curves in figure 6. Grey line: Hyperbola according to the formula $v=22 . S /(555+S)$ 
A similar experiment has been carried out in an Ascent fluorometer. From the initial rates we found the kinetic constants to be $\mathrm{Km}: 280 \mu \mathrm{M}$ and $\mathrm{k}_{\mathrm{cat}} 0,11 \mathrm{~min}^{-1}$.

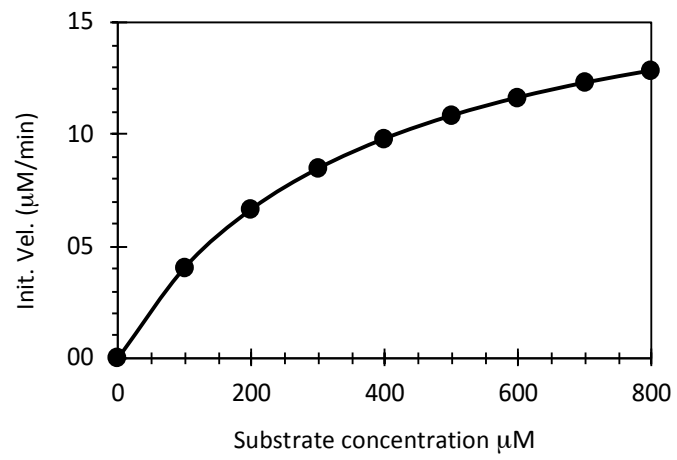

Figure 9. Initial velocity as a function of substrate concentration.

Dots: Experimental points. Line: fitted hyperbola.

Again, plotting the slopes of the lines in figure 4 against the remaining substrate (i.e. the initial substrate concentration minus the product concentration at the point where the slope was calculated) we obtain points that scatter closely around the parabola $v=11 S /(280+S)$ (figure 10$)$.

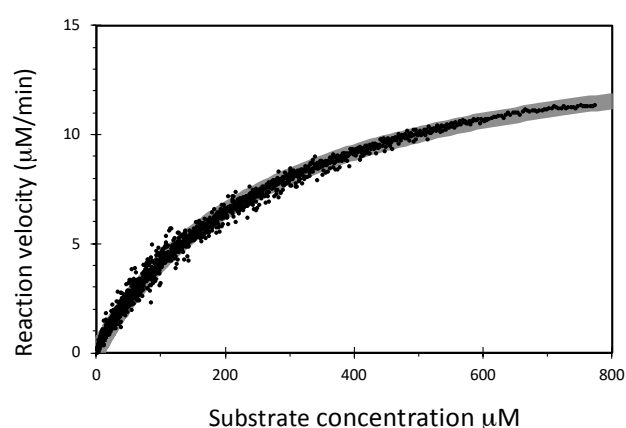

Figure 10. Concentration-reaction velocity plot derived from data in figure 4.

Black points: concentration-velocity pairs from the seven curves in figure 2. Grey line: Hyperbola according to the formula $v=76 . S /(1200+S)$

In figure 10, like in figure 8 the points from the curves at different substrate concentrations are entered together. The individual curves follow MichaelisMenten kinetics obtained at different concentrations as illustrated in figure 4, the dots represent the experimental data and the superimposing lines the theoretical progress curves.

\section{The effect of light absorption}

Absorption of the excitation- and emission light by plasma elements other than the fluorophore obviously diminishes the fluorescence yields. We took icteric plasma 
(Gilbert syndrome) and mixed it with NPP in the proportions 4:5, 3:5, 2:5 and 1:5. Figure 11 shows the OD of these mixtures measured against buffer.

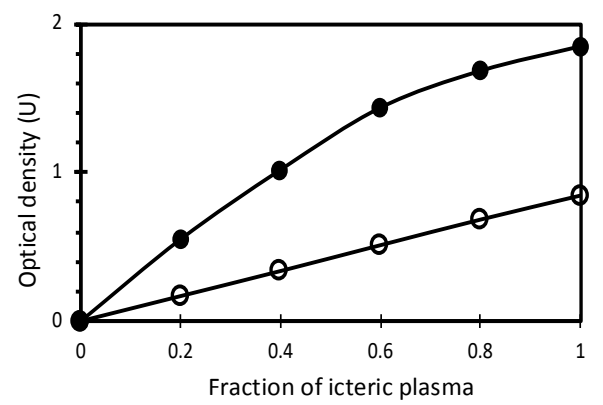

Figure 11. Optical density of a dilution range of icteric plasma in NPP at excitation and emission wavelengths.

$\mathrm{OD}$ measured against normal plasma at $460 \mathrm{nM}$ (black points) and at $390 \mathrm{nM}$ (white points).

When we ran calibrator experiments in these mixtures the results in figure 12 were obtained. It appears that light absorption by the plasma causes a proportional decrease of the signal.

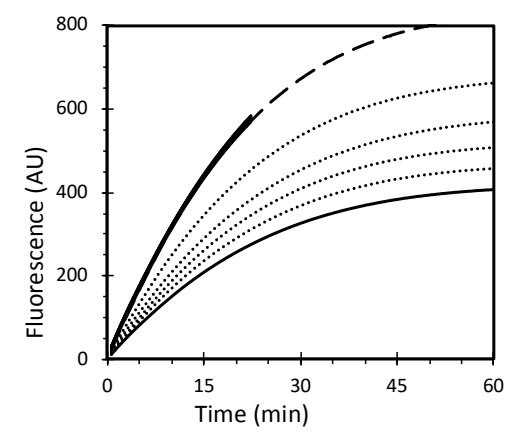

Figure 12. Compensating for colour differences between plasmas.

Upper dashed line: NPP. Lower drawn line: icteric plasma. Intermediate, dotted lines 4:5, 3:5, 2:5 and 1:5 mixtures of icteric and normal plasma. Drawn lines around the upper dashed line: icteric plasma and mixtures normalized to the initial rate of the normal plasma, up to $80 \%$ of the maximum fluorescence.

\section{Discussion}

Apart from the concentration of fluorescent product the fluorescent signal is dependent on three other phenomena:

The inner filter effect (IFE). The relation between the concentration of fluorescent product and the intensity of emitted light is not linear because fluorescent molecules in the solution adsorb both excitation and emission-light. The velocity of 
substrate conversion, as read from fluorescence increase, will therefore be underestimated proportionally (not linearly!) to the amount of fluorescent product that increases as the substrate is converted during the course of the TGexperiment.

Substrate consumption. The velocity of an enzymatic reaction is determined by the concentration of enzyme as well as by the concentration of substrate, so the same enzyme concentration will cause increasingly lower reaction velocities as substrate is consumed in the course of the experiment.

Optical properties of the plasma. The same concentration of fluorescent product measured in different plasmas, at equal excitation intensity, will not show the same emission intensity because plasmas may differ as to optical density at the excitation and emission wavelength and the presence of substances that interact and possibly quench the fluorescence of the product.

These three complications cause every individual plasma to have a different relation between measured reaction velocity and thrombin concentration, at every level of fluorescence. If these effects are not compensated for, then, the persistent $\alpha 2$ macroglobulin-thrombin $(\alpha 2 \mathrm{M}-\mathrm{T})$ complex that forms during any TGexperiment, will eventually consume all substrate and zero activity is measured (see e.g. ${ }^{5}$ ) not because there is no enzymatic activity any more but because the substrate is exhausted. From such curves one cannot calculate what part of the enzymatic activity is due to free thrombin and what to $\alpha 2 \mathrm{M}-\mathrm{T}$ formed during the reaction (see further ${ }^{2}$ ). Therefore, the ETP i.e. the area under the curve of free thrombin cannot be estimated. Moreover, the peak thrombin generation is overestimated by the amount of $\alpha 2 \mathrm{M}$-thrombin then formed. As it is in the beginning of the reaction the amount of activity will be modest and proportional to the area under the curve at the moment of the peak. This explains that with this makeshift approach valid and valuable results can still be obtained if only the peak value is used (e.g. ${ }^{6}$ )

In calibrated automated thrombography (CAT) the formation of fluorescence product in the sample well is run in parallel with a calibration experiment in the same plasma ${ }^{1,7}$. The latter shows the reaction velocity at a fixed known thrombin concentration at every level of fluorescence in that specific plasma. This, in principle copes with all three problems, but it appeared that the comparison between curves is in itself an important source of error (chapter 4).

An apparently different approach to calibration is used in the STGenesia laboratory automaton recently launched by Diagnostica Stago ${ }^{8}$. Here, the fluorescence intensity from a known fixed amount of fluorescent product is measured in a separate sample. In this way differences in fluorescence yield between samples are 
equalled out. Subsequently all samples can be compared to a reference experiment in normal (pooled) plasma in which the course of free thrombin is determined as in the CAT method. This approach has the advantage that no reaction has to be run in the calibration sample but relies on the silent assumption that enzyme kinetic constants $\left(\mathrm{Km}, \mathrm{k}_{\mathrm{cat}}\right)$ are comparable between plasmas, which remains to be proven. Both techniques have the drawback that two samples are required instead of one, albeit for a static measurement in the STGenesia. Another drawback common to both methods in their present embodiment, is that the calculations are not based on the physics and chemistry that govern the IFE and substrate consumption, but rather on algorithms that make the calibrator trace appear independent of IFE and SC which thereafter are applied to the trace from the sample. Moreover, a lack of transparency in the commercial embodiments of these techniques hide these algorithms making the causes for their errors difficult to be traced.

In this chapter we have tried to find the explanation of the observed variability of the calibration factor in the course of the experiment and between plasmas in terms of the proposed confounders (SC and IFE) in the hope to eventually be able to correct for them on basis of the underlying causes rather than by an ad hoc algorithm.

\section{The inner filter effect}

While optical density varies linearly with the concentration of the light adsorbing product over a broad range this is not the case with fluorescence. Molecules near to the source of excitation light adsorb that light and thus shield molecules deeper in solution from being exited (Image 1). Likewise, light emitted from molecules deep in the solution will risk being adsorbed before reaching the detector. The inner filter effect therefore is dependent upon the optical density caused by the fluorescent molecule at both the excitation and emission wavelength. When the fluorescent molecule, measured along the light path of the excitation- and emission light, causes an $\mathrm{OD}$ at the excitation and emission wavelength that sum up to $>0.040$, the inner filter effect will start to play a role ${ }^{3}$.

As can be most easily appreciated from the law of Lambert-Beer: $A=\log _{10}\left(\frac{I_{0}}{I}\right)=$ E.I.c ${ }^{9}$, the inner filter effect is thus dependent upon the concentration of the fluorophore (c) and the optical path length (I), the constant $(\varepsilon)$ cannot be influenced. The easiest way to eliminate the IFE therefore is to use a setup with a low optical pathlength and low concentrations of fluorophore. When the reaction takes place in a soaked filter paper, as in the whole blood TG measurement ${ }^{10}$, the light-path is so short that the inner filter effect does not play a role. It is also possible to lower the substrate concentration. The currently used concentration of 
$417 \mu \mathrm{M}$, results from the seminal publication ${ }^{7}$, where the highest concentration was used that solubility allowed. As will be discussed in chapter 8 , recent insights show that, and why, lower concentrations may serve equally well.

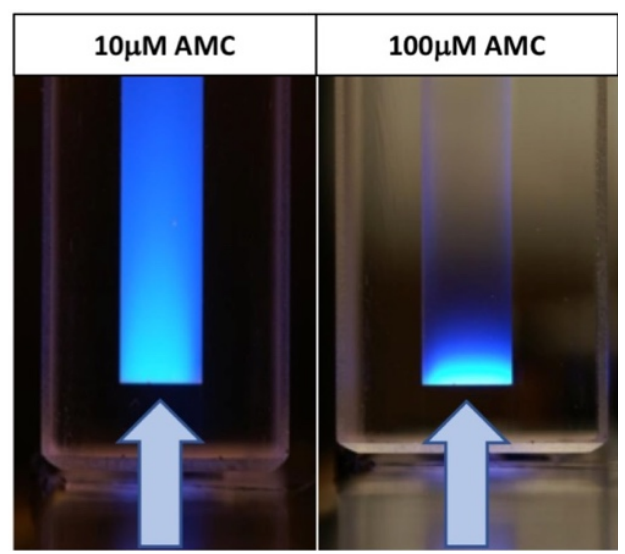

Image 1. The inner filter effect.

It is seen that the higher concentration of AMC adsorbs the incident light and consequently emits less fluorescence.

The relation between fluorescence and the concentration of the fluorescent molecule (C) is given by formula (1): $\mathrm{F}=A C e^{-b C}$, where " $\mathrm{A}$ " is an instrument dependent constant and " $b$ " a constant that is proportional to the specific optical density of the fluorophore. Using this relation, one can calculate the ideal fluorescence $\left(F_{\text {ideal }}\right)$ that would result if no inner filter effect would be present, but this requires use of the product-log function (also known as the Lambert-W function) that is not readily available in common spreadsheets.

In this chapter we show that in the range of fluorescence values used for obtaining a thrombogram, the formula above can be replaced by $F_{\text {exp }}=F_{\max }(1-$ $\left.e^{-k C}\right)$, which allows elimination of the IFE by the formula $F_{\text {ideal }}=-F_{\text {max }} \ln \left(1-F_{\text {exp }} / F_{\text {max }}\right)$. As shown this way of eliminating the IFE works in any fluorometer of this type after normalization of the fluorescence intensity at $300 \mu \mathrm{M}$ AMC to $1000 \mathrm{AU}$ and using 2090 as the value of $F_{\max }$. Similarly, 2.09 times the fluorescence intensity at $300 \mu \mathrm{M}$ AMC can be used for $F_{\text {max }}$. Moreover, from experiments as in figures 3 and 5, the ratio between corrected fluorescence intensity and product concentration is found, which allows for the use of real concentrations in the enzyme kinetic evaluation of an experiment.

$F_{\text {max }}$ thus, is an instrument-dependent constant and can be applied to any experiment used in that instrument, provided that the form of the sample holder, the volume of sample and the excitation light source in it, remain constant. 
The formula used here for converting experimental into ideal values is not the exact solution but can safely be applied to the range of fluorescence values encountered in practice. At concentrations of fluorophore that are (much) higher than those used in practice, fluorescence will reach an optimum and decrease again, as predicted by the formula $F_{\text {exp }}=A C e^{-b C}$.

In summary: The non-proportionality of fluorescence caused by the IFE can be restored to proportionality by a simple mathematical operation involving a constant that is instrument dependent.

\section{Substrate consumption}

Our experiments show that the conversion of substrate by $\alpha_{2} \mathrm{M}-\mathrm{T}$ can be described by the classical Michaelis-Menten parabola $\frac{d P}{d t}=E \cdot k_{c a t} . \mathrm{S} /(\mathrm{Km}+\mathrm{S})$ Where $\mathrm{V}_{\max }$ is the maximal velocity at infinite substrate concentration and $K_{M}$ the concentration at which $\frac{d P}{d t}=\frac{1}{2} V_{\max }$. The fact that all reactions measured during the course of an experiment, i.e. in the presence of (sometimes considerable concentrations of) product, fit in the same hyperbola as that measured from initial rates in the absence of significant product (figures $7 \& 9$ ), shows that there is no, or at least a negligible, inhibition by excess product, an otherwise common phenomenon in enzyme kinetics. This entails that, once the IFE has been corrected for, we can calculate the relation between reaction velocity and enzyme concentration as $E=\frac{d P}{d t}(\mathrm{Km}+\mathrm{S}) /\left(k_{c a t}\right.$. S) i.e. independent of a calibrator curve, as soon as the kinetic constants $\mathrm{Km}$ and $k_{\text {cat }}$ are known.

At the start of the experiment, up to the moment that $55-66 \%$ of the substrate has been consumed the progress curve can be fitted by a parabola (figure 6) (see chapter 6 ). The parabolic relation shows that the reaction velocity is proportional to the substrate concentration, i.e. that the reduced form of the Michaelis-Menten formula applies, i.e. $\frac{d P}{d t}=E . S$. $k_{c a t} / \mathrm{Km}$. To calculate $E$ from $\frac{d P}{d t}$ a single constant, $k_{\text {cat }} / \mathrm{Km}$, suffices.

\section{Plasma colour}

The detection of fluorescence, although seemingly straightforward, is highly dependent on the optical density (OD) of the solution that is being measured. The magnitude of the OD is described by the law of Lambert-Beer: $A=\log _{10}\left(\frac{I_{0}}{I}\right)=$ E.I. ${ }^{9}$. This formula illustrates that the OD is dependent on a constant: $\varepsilon$, also known as the molar attenuation coefficient, the path length (I) and the concentration (c). The colour of the plasma relates to the constant known as the 
molar extinction coefficient in this formula, it is an intrinsic property of the substrate and the constituents of the plasma. As has been observed variation in the optical density of the plasma can be as large as $10 \%$, justifying the need for a correction factor. Luckily as can be appreciated from the Lambert-Beer formula the correction is rather straight forward as only the change in molar attenuation coefficient has to be compensated for, making the solution not more than a simple multiplication.

\section{In conclusion}

Substrate consumption and inner filter effect, that have been held responsible for the non-linearity of the fluorescence curve resulting from a fixed enzymatic activity have been shown to indeed explain this phenomenon. A formula has been found to compensate for the inner filter effect. This allows to transform any fluorescence trace into a trace of formed product, hence the remaining substrate concentration at any point in time is known. It has also been shown that Michaelis-Menten kinetics apply to the substrate consumption process. This means that from a fluorescence trace the concentration of enzyme can be calculated if the kinetic constants are known. How this can be used to directly calculate the thrombin generation curve from a fluorescence trace without referring to a simultaneous calibrator experiment in the same plasma will be the subject of a forthcoming article. 


\section{References}

1. Hemker, H. C. et al. Calibrated Automated Thrombin Generation Measurement in Clotting Plasma. Pathophysiol. Haemost. Thromb. 33, 4-15 (2003).

2. Hemker, H. C. \& Kremers, R. Data management in Thrombin Generation. Thromb. Res. 131, 3-11 (2013).

3. Lakowicz, J. Principles of Fluorescence Spectroscopy. 1, (2006).

4. Valluri, S. R., Jeffrey, D. J. \& Corless, R. M. Some applications of the Lambert W function to physics. Can. J. Phys. 78, 823-831 (2000).

5. Turecek, P. L. et al. Factor VIII Inhibitor-Bypassing Agents Act by Inducing Thrombin Generation and Can Be Monitored by a Thrombin Generation Assay. Pathophysiol. Haemost. Thromb. 33, 16-22 (2003).

6. Hron, G., Kollars, M., Binder, B. R., Eichinger, S. \& Kyrle, P. A. Identification of patients at low risk for recurrent venous thromboembolism by measuring thrombin generation. JAMA 296, 397-402 (2006).

7. Hemker, H. et al. The calibrated automated thrombogram (CAT): a universal routine test for hyper-and hypocoagulability. Pathophysiol. Haemost. Thromb. 32, 249-253 (2002).

8. Calzavarini, S. et al. Validation of the automated ST Genesia for thrombin generation assay: evaluation of variability and reference ranges in a cohort of healthy donors. in SWISS MEDICAL WEEKLY 148, 29S-29S (EMH SWISS MEDICAL PUBLISHERS LTD FARNSBURGERSTR 8, CH-4132 MUTTENZ, SWITZERLAND, 2018).

9. Swinehart DF. The beer-lambert law. J. Chem. Educ. 39, 333 (1962).

10. Ninivaggi, M. et al. Whole-Blood Thrombin Generation Monitored with a Calibrated Automated Thrombogram-Based Assay. Clin. Chem. 58, 1252-1259 (2012). 


\section{Supplementary information}

Formula 3 has been obtained as follows: Knowing that the first derivative of the function is a straight line it should have the form of the following first order differential equation:

$F_{\text {exp }}^{\prime}=C_{1}-C_{2} \cdot f$

Which can be solved:

$F_{\text {exp }}(t)=-\alpha \cdot e^{-\beta t}+\gamma$

Because the curve is a straight line that passes through the origin:

$F_{\text {exp }}(0)=0->F_{\text {exp }}(t)=\alpha\left(1-e^{-\beta t}\right)$

If we take the first derivative from this function:

$F^{\prime}{ }_{\text {exp }}=\alpha \cdot \beta \cdot e^{-\beta t}$

And we plot the line through the origin:

$F^{\prime} \exp (0)=\alpha \cdot \beta$

We can then take the formula for the ideal fluorescence curve without inner filter effect:

$F_{\text {ideal }}(t)=F^{\prime} \exp (0) \cdot t=\alpha \cdot \beta \cdot t$

When we fill this formula in our original equation we get as a solution the equation that turns our experimental fluorescence into the ideal fluorescence curve.

$F_{\text {ideal }}=-\alpha \cdot \ln \left(1-\frac{F_{\text {exp }}}{\alpha}\right)$

Where $\alpha=F_{\max }$. 

Chapter 7

Indirect Measurement of Initial Reaction Rates from Progress Curves, its Application to Investigate the Influence of Albumin on the Interaction of Thrombin and the $\alpha 2$ macroglobulin-Thrombin Complex With the Fluorogenic Substrate ZGGR-AMC.

Martijn Moorlag, Saartje Bloemen, Piet W. Hemker, Coenraad Hemker. Manuscript in preparation 


\section{Abstract}

Background Modern techniques for measuring thrombin generation (TG) are based on the principle that a slow-acting thrombin specific Z-Gly-Gly-Arg-7-amino4-methylcoumarin (ZGGR-AMC) substrate is added to the clotting plasma. Solubility of higher concentrations of the ZGGR-AMC substrate is only attainable at high $(60 \mathrm{mg} / \mathrm{ml})$ albumin concentrations, this fact suggests that the substrate binds to albumin. Albumin is the most abundant protein in blood plasma. It constitutes nearly two thirds of plasma proteins on a weight basis $3.5-5 \mathrm{~g} / \mathrm{dl}$ on a total protein concentration of $5.9-7.8 \mathrm{~g} / \mathrm{dl}$. Since the final determination of the thrombin concentration in the TG experiment depends on two reactions, the TG and the calibrator, an influence of albumin on both or either of these reactions can be a cause for experimental variation.

Methods TG-like measurements where performed using both thrombin and $\alpha_{2} \mathrm{M}$ $\mathrm{T}$ to cleave the substrate in a buffer system that contained a concentration range of albumin, as well as a concentration range of ZGGR-AMC substrate.

Results Simulation of Michalis-Menten kinetics in the range of constants and substrate concentrations to be expected in our experiments, showed that, for all practical purposes the first $2 / 3$ part of a product formation can be fitted to a parabola. This allows estimation of the initial rate of a reaction from the linear component in a parabolic fit. From such initial rate kinetics, it appeared that has hardly an influence on the kinetic constants of thrombin but a large influence of the $\mathrm{Km}$ of $\alpha_{2} \mathrm{M}-\mathrm{T}$.

Conclusions Initial rate measurement is preferably done by fitting a parabola to the first $2 / 3$ of a progress curve rather than by fitting a tangent to that curve at the origin. It is likely that the substrate binds to albumin and that thrombin can convert both bound and free substrate whereas $\alpha_{2} \mathrm{M}$-T only attacks the free form. 


\section{Introduction}

Modern techniques for measuring thrombin generation (TG) are based on the principle that a slow-acting thrombin specific substrate is added to the clotting plasma ${ }^{1}$. It is essential that sufficient amounts of substrate remain, as long as the experiments last. The concentration of $417 \mu \mathrm{M}$ that is currently being used, stems from the original publication ${ }^{2}$, where the highest soluble concentration was used. Solubility of higher $(>200 \mu \mathrm{M})$ substrate concentrations is only attainable at high $(60 \mathrm{mg} / \mathrm{ml})$ albumin concentrations, provided the exclusion of other solvents e.g. DMSO. This fact already suggests that the Z-Gly-Gly-Arg-7-amino-4methylcoumarin (ZGGR-AMC) substrate used binds to albumin. Binding to albumin poses a number of questions: is the bound substrate still available to be split by thrombin and/or the $\alpha_{2}$-Macroglobulin-Thrombin complex $\left(\alpha_{2} M-T\right)$ ? If not, will albumin-bound substrate serve as a storage-pool for free substrate and therewith reduce the apparent concentration at which half maximal reaction rate is attained, i.e. will it increase the apparent $\mathrm{Km}$ ? If the albumin-bound substrate still can be split, does the binding affect the turnover number $\left(k_{\text {cat }}\right)$ ? Does thrombin behave differently from $\alpha_{2} \mathrm{M}-\mathrm{T}$ in this respect? These are all questions that are of a direct practical interest when measuring TG.

Albumin is the most abundant protein in blood plasma. It constitutes nearly two thirds of plasma proteins on a weight basis $3.5-5 \mathrm{~g} / \mathrm{dl}$ on a total protein concentration of $5.9-7.8 \mathrm{~g} / \mathrm{dl}^{3}$. Albumin is known for its capacity to carry other molecules, notably those with a hydrophobic character such as steroid hormones and fatty acids ${ }^{4}$.

The calibrated automated thrombography (CAT) procedure depends upon the comparison of the activity of thrombin (in the TG experiment) to that of A2M-T in the calibrator. If the ratio of activities is influenced by albumin, the outcome of the comparison will be dependent upon the albumin content of the plasma. Quantitative assessment of the albumin effect - if any - therefore is required to solve the practical question whether it is likely that the variation of the albumin content of a sample can have a significant influence on the outcome of a TG experiment.

This question can in principle be solved by classical initial rate enzyme kinetics. In experiments carried out on a 96-well plate fluorometer, initial rates are notoriously difficult to estimate correctly for different reasons. For one, due to the movement of the plate from the injection site to the measurement site, measurement starts at an unknown moment after the start of the reaction. Another reason is that the first few measurements are often unstable, e.g. due to temperature equilibration. We therefore developed a method to estimate the initial rate from the 
fluorescence trace not from a tangent to the trace at zero time, as is usually done, but by fitting a parabola to the first part of the trace and calculating its linear component.

\section{Materials and methods}

\section{Reagents}

ZGGR-AMC substrate was purchased from Bachem (Bubendorf, Switzerland). Bovine serum albumin (BSA) was obtained from Sigma Aldrich (Saint Louis, Missouri, USA). The $\alpha_{2} \mathrm{M}-\mathrm{T}$ complex was prepared as described previously (1). The BSA containing buffer was prepared with $20 \mathrm{mM}$ Hepes, $0.02 \% \mathrm{NaN}_{3}$ and $60 \mathrm{mg} / \mathrm{ml}$ BSA at pH 7.35 (BSA 60) $)^{5}$. Buffer A contained $20 \mathrm{mM}$ Hepes, $140 \mathrm{mM} \mathrm{NaCl}$ and $0.02 \% \mathrm{NaN}_{3}$ at a pH of 7.35 (which is basically the $5 \mathrm{mg} / \mathrm{ml} \mathrm{BSA}$ buffer used to dissolve reagents in TG experiments, but omitting the BSA). The latter was used to dilute the BSA-containing buffer to the desired concentrations. The ZGGR-AMC substrate was dissolved in dimethyl sulfoxide (DMSO) to a concentration of 100 $\mathrm{mM}$. Further dilutions of the substrate were made using the BSA 60 buffer, whilst correcting to maintain a constant DMSO concentration.

\section{Experimental set-up}

Experiments were performed in an Ascent reader fluorimeter (Thermo Labsystems, Helsinki, Finland) using an experimental set-up resembling TG assays. Thrombin and $\alpha_{2} \mathrm{M}$-thrombin were added $(20 \mu \mathrm{l})$ in final concentrations between 0 and 100 nM. BSA concentrations were $15,30,40,50$ and $60 \mathrm{mg} / \mathrm{ml}$ (concentration in $2 / 3$ of final volume, i.e. $80 \mu \mathrm{l}$ ). ZGGR-AMC was added in final concentrations of between 50 and $417 \mu \mathrm{M}(20 \mu \mathrm{l})$. The reagents were incubated for $10 \mathrm{~min}$ at $37^{\circ} \mathrm{C}$ and after addition of the substrate, followed by $5 \mathrm{~s}$ of shaking, the measurement was initiated.

\section{Inner filter effect correction}

Fluorescence data were corrected for the inner filter effect via the formula: $F_{\text {corrected }}=-3000 \ln \left(1-\frac{F_{\text {exp }}}{3000}\right) ; 3000$ being the correction factor specific for the fluorimeter used (see chapter 6). From the corrected levels after complete conversion of the substrate the ratio between product concentration and fluorescence was calculated to be 6.29. The kinetic constants were determined from the initial reaction rates using the direct linear plot according to Eisenthal and Cornish-Bowden ${ }^{6}$. 


\section{Results}

Theory and numerical experiments

Assuming Michealis-Menten enzyme kinetics, the course of product formation in an experiment in which a given, stable concentration of enzyme $(E)$ acts on a given, known amount of added substrate $\left(S_{0}\right)$ is given by the formula:

$\frac{d P}{d t}=k_{c a t} E\left(S_{0}-P(t)\right) /\left(K_{m}+S_{0}-P(t)\right)$

The solution of this equation involves the product-log or Lambert-W function, since this function is not readily available in spreadsheets the possibility of its application in practice remains to be desired.

Conventional initial rate enzyme kinetics circumvents this problem by choosing experimental conditions such that product formation can be neglected, in which case formula A reduces to:

$\frac{d P}{d t}=k_{c a t} E\left(S_{0}\right) /\left(K_{m}+S_{0}\right)$

Due to the instability that is often observed in the first minutes of an experiment in a 96-well plate fluorimeter, and because of the delay in recording the experiment due to dispensing and shaking, this approach is less suited to implement in this type of experiment; which however is the mainstay of the TG measurement.

To circumvent this problem, we reasoned that after the unmeasurable initial rate there has to be a phase in which the velocity decreases proportionally to the amount of product formed. The reason is that in the beginning of an experiment the numerator of formula $\mathrm{A}: k_{\text {cat }} E\left(S_{0}\right)-P(t)$ decreases proportionally with $\mathrm{P}(\mathrm{t})$, but the denominator: $K_{m}+S_{0}-P(t)$ does not.

As long as: $P(t) \ll K_{m}+S_{0}$ the effect of product formation on the denominator can be neglected and formula $A$ reduces to:

$\frac{d P}{d t}=k_{c a t} E\left(S_{0}-P(t)\right) /\left(K_{m}+S_{0}\right)$

As long as: $P(t) \ll K_{m}+S_{0}$ the velocity of the reaction will be uniformly decelerated, much like motion under gravity, but decreasing instead of increasing. This means that the linear component of a parabola fitted to the progress curve of product will give the initial rate. The progress of product formation then is given by:

$P(t)=1 / 2 S_{0} H^{2} t^{2}+S_{0} H t+C$, where $H=\left(V /\left(K_{m}+S_{0}\right)\right)$ 
A fluorescence-time curve in which the inner filter effect does not play a role or has been corrected for, will represent product formation but for a factor $\kappa$, the number of arbitrary fluorescence units per $\mu \mathrm{M}$ of product.

When we fit a parabola $\left(F=-a^{2} t+b t+c\right)$ to the fluorescence-time curve we will find $a=1 / 2 \mathrm{~S}_{0} \mathrm{H}^{2} . \kappa$ and $b=\mathrm{S}_{0} \mathrm{H} \kappa$. The third constant $c$ is the level of fluorescence at $\mathrm{t}=0$.

In order to see in how far the product progress curve is represented by a parabola, we constructed such curves by simple numerical integration in a spreadsheet (Table 1) 
Table 1. Spreadsheet for the numerical calculation of the course of product.

(The IF statement for the remaining substrate prevents negative substrate concentrations.)

\begin{tabular}{|c|c|c|c|c|}
\hline A & $\mathrm{B}$ & $\mathrm{C}$ & $\mathrm{D}$ & $\mathrm{E}$ \\
\hline \multicolumn{5}{|l|}{1} \\
\hline 2 & Enzyme = & 100 & & \\
\hline 3 & $\mathrm{Km}=$ & 150 & & \\
\hline 4 & $\mathrm{k}_{\mathrm{cat}}=$ & 0,08 & & \\
\hline 5 & $\begin{array}{l}\text { Initial } \\
\text { substrate= }\end{array}$ & 150 & & \\
\hline 6 & Time step= & 0,5 & & \\
\hline 7 & Time & Substrate & Product & Reaction velocity \\
\hline 8 & 0 & $=\mathrm{C} 5$ & $\begin{array}{l}=(C \$ 6- \\
c 9)^{*} \mathrm{C} \$ 6\end{array}$ & $=(C \$ 3 * C \$ 5 * C 9 /(C \$ 4+C 9))$ \\
\hline 9 & 0,5 & $\begin{array}{l}=I F((C 9- \\
E 9)<0 ; 0 ;(C 9-E 9))\end{array}$ & $\begin{array}{l}=(C \$ 6- \\
C 10)^{*} C \$ 6\end{array}$ & $=(C \$ 3 * C \$ 5 * C 10 /(C \$ 4+C 10))$ \\
\hline 10 & 1 & $\begin{array}{l}=I F((C 10- \\
E 10)<0 ; 0 ;(C 10- \\
E 10))\end{array}$ & $\begin{array}{l}=(C \$ 6- \\
c 11)^{*} \mathrm{C} \$ 6\end{array}$ & $=(C \$ 3 * C \$ 5 * C 11 /(C \$ 4+C 11))$ \\
\hline 11 & 1,5 & $\begin{array}{l}=I F((C 11- \\
E 11)<0 ; 0 ;(C 11- \\
E 11))\end{array}$ & $\begin{array}{l}=(C \$ 6- \\
c 12)^{*} \mathrm{C} \$ 6\end{array}$ & $=(C \$ 3 * C \$ 5 * C 12 /(C \$ 4+C 12))$ \\
\hline 12 & 2 & $\begin{array}{l}=I F((C 12- \\
E 12)<0 ; 0 ;(C 12- \\
E 12))\end{array}$ & $\begin{array}{l}=(C \$ 6- \\
C 13)^{*} \mathrm{C} \$ 6\end{array}$ & $=(C \$ 3 * C \$ 5 * C 13 /(C \$ 4+C 13))$ \\
\hline 13 & 2,5 & $\begin{array}{l}=I F((C 13- \\
E 13)<0 ; 0 ;(C 13- \\
E 13))\end{array}$ & $\begin{array}{l}=(C \$ 6- \\
C 14)^{*} \mathrm{C} \$ 6\end{array}$ & $=(C \$ 3 * C \$ 5 * C 14 /(C \$ 4+C 14))$ \\
\hline 14 & 3 & $\begin{array}{l}=I F((C 14- \\
E 14)<0 ; 0 ;(C 14- \\
E 14))\end{array}$ & $\begin{array}{l}=(C \$ 6- \\
C 15)^{*} C \$ 6\end{array}$ & $=(C \$ 3 * C \$ 5 * C 15 /(C \$ 4+C 15))$ \\
\hline 15 & 3,5 & $\begin{array}{l}=I F((C 15- \\
E 15)<0 ; 0 ;(C 15- \\
E 15))\end{array}$ & $\begin{array}{l}=(C \$ 6- \\
c 16)^{*} \mathrm{C} \$ 6\end{array}$ & $=(C \$ 3 * C \$ 5 * C 16 /(C \$ 4+C 16))$ \\
\hline 16 & 4 & $\begin{array}{l}=I F((C 16- \\
E 16)<0 ; 0 ;(C 16- \\
E 16))\end{array}$ & $\begin{array}{l}=(C \$ 6- \\
c 17)^{*} \mathrm{C} \$ 6\end{array}$ & $=(C \$ 3 * C \$ 5 * C 17 /(C \$ 4+C 17))$ \\
\hline 17 & etc. & etc. & etc. & etc. \\
\hline
\end{tabular}




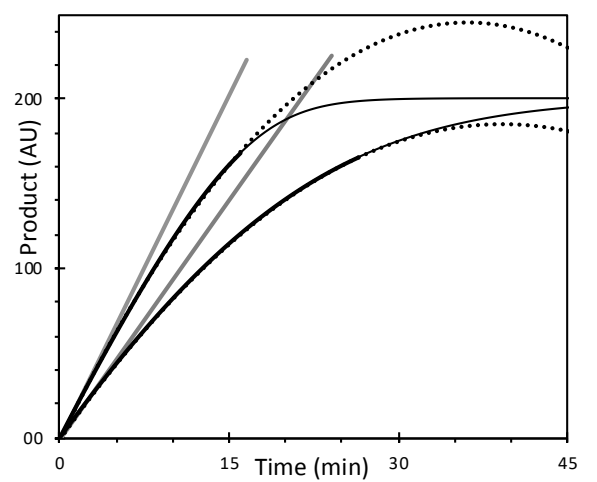

Figure 1. Model of parabolic fit.

Thin lines: Course of product. Thick lines: First two-thirds of the product course. Dotted lines: Parabolic fit to the first two-thirds of the product curve. Grey Lines: Idealized product curves (see text) $\mathrm{Km}=50$ (upper curve) and 150 (lower curve) $\mathrm{E}=100, \mathrm{~S}_{0}=200, \mathrm{k}_{\text {cat }}=0.08$.

In figure 1 two such simulations are shown together with the parabolic fit to the product line until $2 / 3$ of the substrate has been converted. To judge the goodness of fit we made plots of the product values against the parabolic fit (figure 2). It is seen that up to a level where $67 \%$ of the substrate is converted the two curves are for all practical purposes identical.
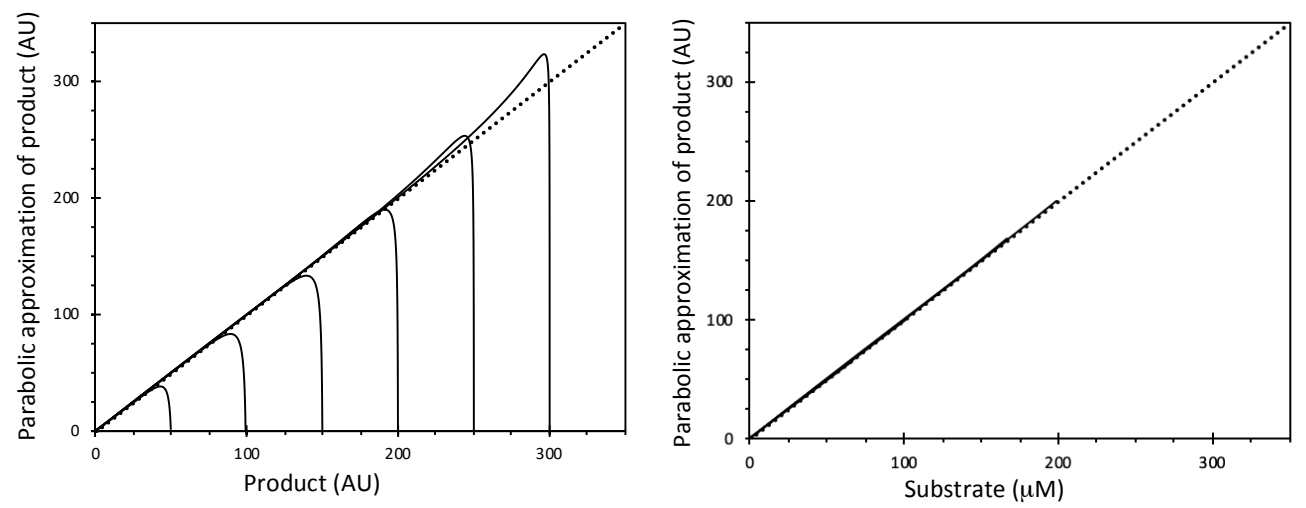

Figure 2. Goodness of fit of the parabolic approximation.

The simulated product curves are plotted against their parabolic approximations. Left frame: Complete range of product values. Right frame: Product values up till $2 / 3$ of the substrate is consumed.

We repeated this numerical experiment for a large number of combinations of the four variables and it turned out to be invariant for $k_{\text {cat }}$ and enzyme concentration and for the ratio of initial substrate concentration to $\mathrm{Km}$.

The next question is how to "straighten up" a progress curve, i.e. how to convert the actual product concentrations $\left(P_{\exp }\right)$ into product concentrations that would 
have been observed if substrate consumption would not have played a role ( $\left.P_{\text {ideal }}\right)$. This is an important question because the transition $\mathrm{P}_{\exp } \rightarrow \mathrm{P}_{\text {ideal }}$ can be applied to any experimental fluorescence trace in which the enzyme concentration varies in time (e.g. a TG curve). The result is an ideal trace, of which the first derivative is proportional to the course of enzyme (thrombin) concentration; Provided that the inner filter effect does not play a role or is corrected for. The formula to bring about this transition is:

$F(t)_{\text {ideal }}=\frac{2 F(t)_{\text {exp }}}{1}+\left(\operatorname{Sqrt}\left(1+2 \mathrm{HF}(t)_{\text {exp }}\right)\right)$ with $H=V /\left(K_{M}+S_{0}\right) \mathrm{E}$

In figure 1 the grey lines represent the simulated product curves after transformation with this formula.

\section{Biochemical experiments}

We determined the course of fluorescence at 50,100, 200, 300 and $417 \mu \mathrm{M}$ of ZGGR-AMC in buffer $A$ in a range of albumin concentrations: 15, 30, 40, 50 and 60 $\mathrm{mg} / \mathrm{ml}$, using thrombin $(100 \mathrm{nM})$ and $\alpha 2 \mathrm{M}-\mathrm{T}$ complex $(175 \mathrm{nM})$ as the enzymes. Figure 3 shows the experimental curves at $417 \mu \mathrm{M}$ substrate in their original form (left frame), with $2 / 3$ of the substrate converted together with the fitted parabolas (middle frame) and after correction for the curvature using formula D. We checked that the initial velocities were proportional to the enzyme concentrations over a range of $10-300 \mathrm{nM}$ (results not shown). It is seen that parabolas fit perfectly until $2 / 3$ of the substrate has been converted, as predicted by theory.
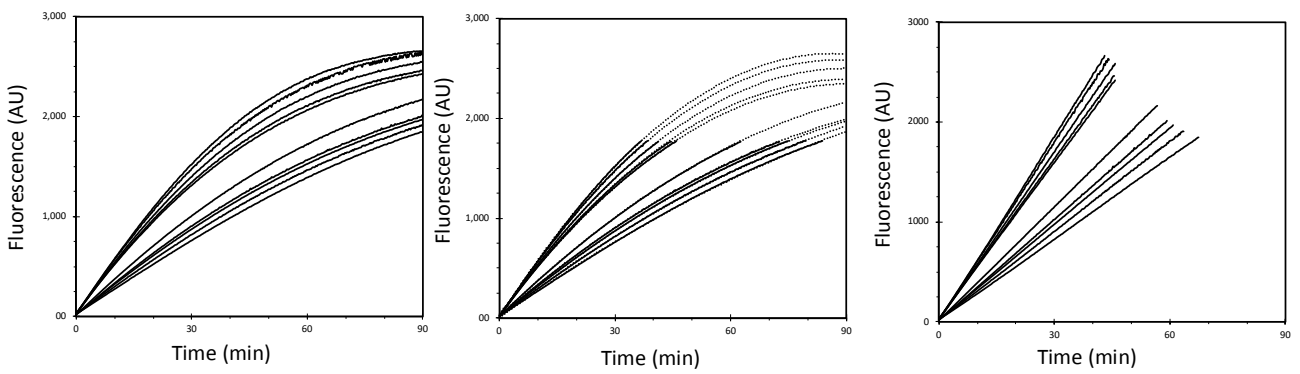

Figure 3. Fluorescence curves at $417 \mu \mathrm{M}$ substrate.

Left frame: Raw experimental data; middle frame $2 / 3$ of the experimental curves with fitted parabolas. right frame: data "idealized" with formula D. Upper bundle: $\alpha_{2} \mathrm{M}-\mathrm{T}$ at 175 $\mathrm{nM}$. Lower bundle: Thrombin at $100 \mathrm{nM}$. In each bundle the albumin concentrates were $15,30,40,50$ and $60 \mathrm{mg} / \mathrm{ml}$ (top to bottom).

The initial velocities thus found, expressed in $\mu \mathrm{M}$ substrate per $\mathrm{nM}$ enzyme per minute, are presented in table 2 . 
Table 2. Initial velocities of substrate conversion.

\begin{tabular}{|c|c|c|c|c|c|c|}
\hline $\begin{array}{l}\alpha_{2} M- \\
\text { Thrombin }\end{array}$ & 15 & 30 & 40 & 50 & 60 & $\begin{array}{l}\text { Albumin } \\
\text { (mg/ml) }\end{array}$ \\
\hline $\begin{array}{l}\text { Substrate } \\
(\mu \mathrm{M})\end{array}$ & \multirow[b]{2}{*}{14.36} & \multirow[b]{2}{*}{12.47} & \multirow[b]{2}{*}{11.36} & \multirow[b]{2}{*}{10.22} & \multirow[b]{2}{*}{9.31} & \\
\hline 50 & & & & & & \\
\hline 100 & 25.62 & 22.64 & 20.92 & 19.02 & 17.70 & \\
\hline 200 & 42.27 & 38.69 & 35.66 & 32.66 & 30.74 & \\
\hline 300 & 51.51 & 48.74 & 46.42 & 43.62 & 41.59 & \\
\hline 417 & 61.01 & 58.58 & 55.06 & 52.38 & 50.72 & \\
\hline \multicolumn{7}{|l|}{ Thrombin } \\
\hline $\begin{array}{l}\text { Substrate } \\
(\mu \mathrm{M})\end{array}$ & 15 & 30 & 40 & 50 & 60 & $\begin{array}{l}\text { Albumin } \\
(\mathrm{mg} / \mathrm{ml})\end{array}$ \\
\hline 50 & 8.09 & 7.06 & 6.86 & 6.67 & 6.40 & \\
\hline 100 & 14.70 & 12.64 & 12.47 & 12.06 & 11.71 & \\
\hline 200 & 24.50 & 21.84 & 20.93 & 20.01 & 19.62 & \\
\hline 300 & 31.35 & 28.32 & 25.36 & 27.25 & 24.52 & \\
\hline 417 & 37.66 & 33.57 & 32.13 & 29.88 & 27.54 & \\
\hline
\end{tabular}

From the data in table 2 we calculated $\mathrm{Km}$ and $\mathrm{k}_{\text {cat }}$ both for thrombin and $\alpha_{2} \mathrm{M}-\mathrm{T}$ (Table 3 and figure 4). As can be appreciated from the table, albumin hardly has an influence on the kinetic constants of thrombin but a large influence of the $\mathrm{Km}$ of $\alpha_{2} \mathrm{M}-\mathrm{T}$.

Table 3. Kinetic constants of $\alpha_{2} M-T$ and thrombin.

\begin{tabular}{|l|l|l|l|l|l|}
\hline Albumin $(\mathrm{mg} / \mathrm{ml})$ & 15 & 30 & 40 & 50 & 60 \\
\hline$\alpha_{2} \mathrm{M}-\mathrm{T} \mathrm{Km}(\mu \mathrm{M})$ & 327 & 421 & 464 & 541 & 632 \\
\hline$\alpha_{2} \mathrm{M}-\mathrm{T} \mathrm{kat}_{\text {cat }}\left(\mathrm{m} \mathrm{in}^{-1}\right)$ & 1.73 & 1.87 & 1.87 & 1.92 & 2.04 \\
\hline $\begin{array}{l}\text { Thrombin Km } \\
(\mu \mathrm{M})\end{array}$ & 408 & 446 & 417 & 392 & 352 \\
\hline $\begin{array}{l}\text { Thrombin k } \\
\left(\mathrm{min}^{-1}\right)\end{array}$ & 1.18 & 1.11 & 1.02 & 0.94 & 0.83 \\
\hline
\end{tabular}



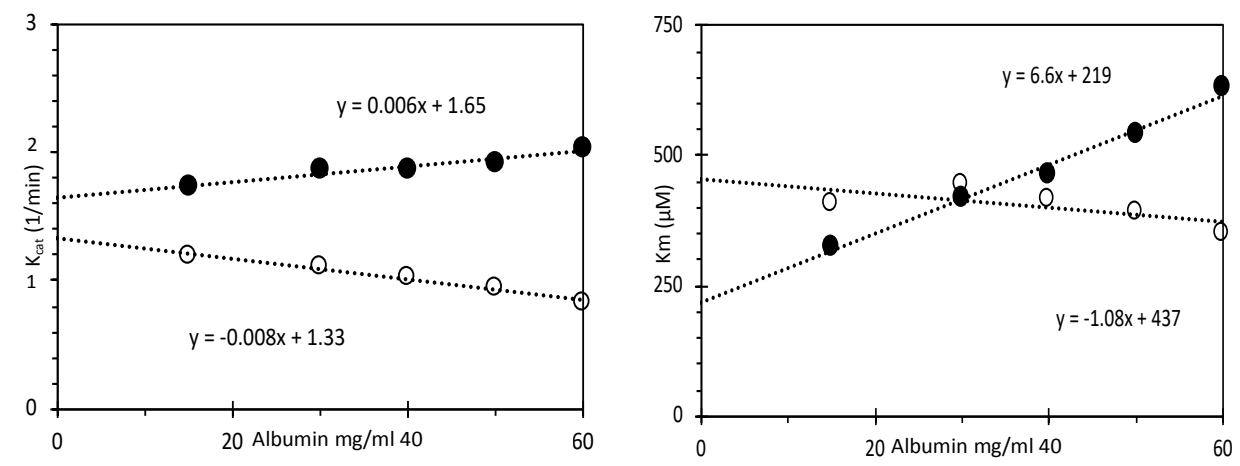

Figure.4. The influence of albumin on the kinetic constants of substrate conversion by thrombin and by $\alpha_{2} M-T$.

Formula $D$ predicts that the square of the linear coefficient $\left(b=S_{0} H\right)$ divided by the quadratic coefficient $\left(a=\mathrm{S}_{0} \mathrm{H}^{2}\right)$ will be equal to $\mathrm{S}_{0}$. As a check on the developed theory we calculated $S_{0}$ as $b^{2} / 2 a$ (see formula $D$ above) and compared it to the known added values of $\mathrm{S}_{0}$ (figure.5).

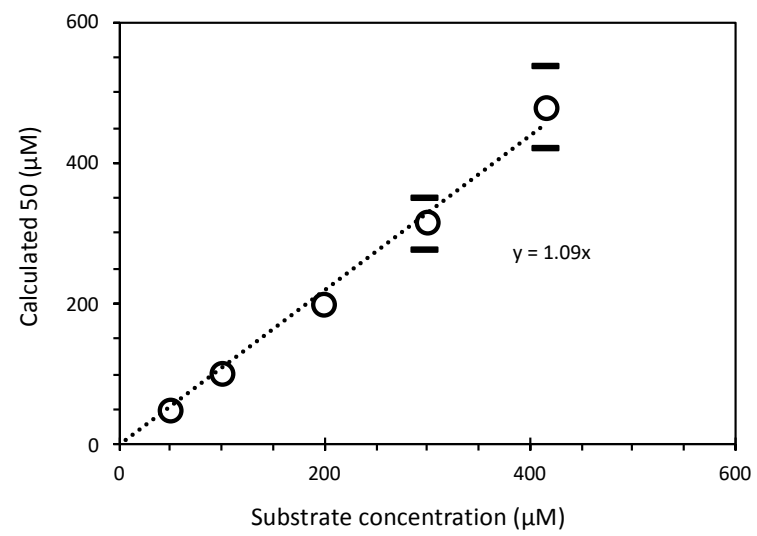

Figure 5. Added and calculated values of $S_{0}$.

The added concentrations of substrate (abscissa) are plotted against the concentrations calculated on basis of the fitted parabola (see text). The horizontal lines indicate 1XSD. When not shown they approached closely the limits of the circle. 
Figure 6 displays the values of $\mathrm{H}$ for thrombin and the corresponding ones for $\alpha_{2} \mathrm{M}-\mathrm{T}$ at all substrate- and albumin concentrations.

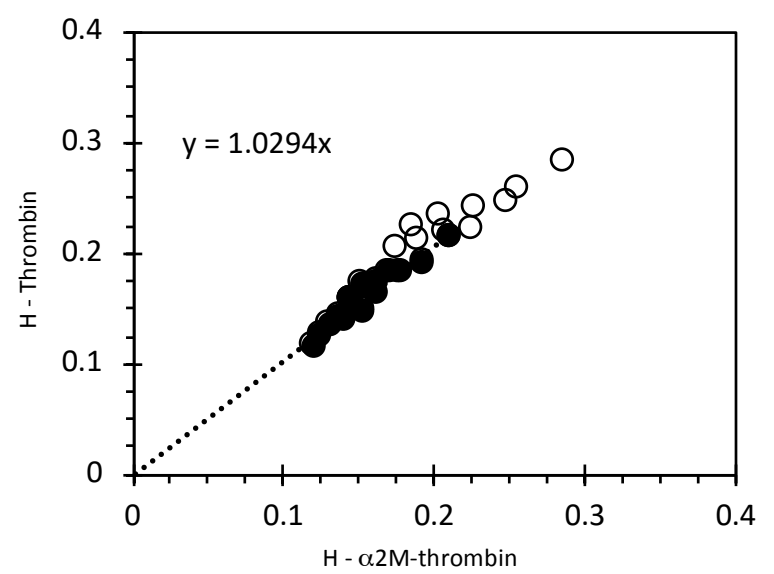

Figure 6. Comparison of the correction constants (H) of thrombin and $\alpha 2 M$-thrombin. Open points: Albumin $<200 \mathrm{mg} / \mathrm{ml}$. Closed points: Albumin $>100 \mathrm{mg} / \mathrm{ml}$

\section{Discussion}

In this article we studied the effect of albumin on the kinetic constants of the conversion of the ZGGR-AMC substrate, in doing so we noticed that measuring a correct initial velocity in a 96 well setup is difficult due to the instability of the fluorescence trace during the first few minutes of the measurement. Realising that this also must be a serious source of error when calibrating TG-curves, we developed an initial rate measurement on basis of a parabolic fit to the initial part of the curve.

In TG measurement the main causes for experimental errors are a: inadequate calibration procedures and $b$ : the combination of effects, among which temperature, that influence the initial rate found in the measurement rendering it unrepresentative for the kinetics of the remainder of the process (see chapter 4). Estimating the correct initial rate of the calibrator is a key procedure in all calculations necessary to establish exact thrombin concentrations. We therefore wanted to establish a more reliable method to calculate initial rates.

We made use of the fact that substrate consumption although it affects both the numerator and the denominator of the Michaelis-Menten formula (formula A), it is only the numerator that diminishes proportionally with the remaining substrate. The change in the numerator is much smaller because there, an invariable large constant $(\mathrm{Km})$ is added to the diminishing substrate concentration. If the change in the denominator is neglected, the formulas predict a parabolic progress curve. In order to see to which extent this approximation can be used in practice, we 
performed numerical simulation experiments over a large range of kinetic constants and substrate concentrations and found that this can be used at least during the first $2 / 3$ of substrate consumption. In practice the TG experiment will be finished already before this level of substrate consumption is reached.

In order to measure the influence of albumin on the conversion of the substrate by both thrombin and $\alpha_{2} \mathrm{M}-\mathrm{T}$, plasma could not be used. Firstly, because it contains a high concentration of albumin and secondly because of the presence of antithrombins, therefore we used an albumin containing buffer instead. Additionally, this allowed us to investigate the influence of albumin on the kinetics of substrate conversion at the same time. We show that experimental curves, in the range below $67 \%$ substrate consumption, indeed can be faithfully represented by a fitted parabola: $P=a t^{2}+b t+c$. In this fit $c$ is the non-zero start of the curve, due to the fact that in this type of experiments the start of the reaction does not exactly coincide with the start of the experiment. Whether $\mathrm{c}$ is subtracted from the observed fluorescence value or not does not influence the calculations.

Logically one should discard the first minutes of the experimental trace because this is the part that is most disturbed and it renders direct measurement of the initial velocity dubious. In practice we saw that this does not significantly influence the values of the quadratic- and the linear parameters ( $a$ and $b$ ). When, in a next article, we will apply this technique to the calibration of TG curves, we will use only that part of the calibrator curve that covers the time and fluorescence values of the TG-curve, i.e. the part that starts after the lag-time of TG and that reaches up to the highest level of fluorescence attained by the TG experiment.

Theory shows that the quadratic and the linear parameters are closely related:

$a=\frac{1}{2} S_{0} H^{2}$. and $b=S_{0} H$. From this it follows that $\frac{b^{2}}{2 a}=S_{0}$. This we checked and found a linear relationship, but with the calculated values being $9 \%$ higher (figure 5). We attribute this to an unknown second order effect and find the difference not big enough to refute the underlying theory, that, we recall, remains an approximation.

Additionally, we demonstrate that the formula that we developed to correct for substrate consumption indeed renders perfectly straight lines, i.e. a reaction velocity identical to the initial rate. Previously, we proposed the formula $F_{\text {ideal }}=$ . $\operatorname{arctanh}\left(\frac{F_{\text {exp }}}{}\right)$ to compensate for both the inner filter effect and substrate consumption ${ }^{5}$. The procedure requires an educated guess of the constant $\alpha$. In practice that $\alpha$ is used that renders the calibrator curve a straight line, which involves a trial and error procedure or a mathematical optimization program. This 
is a weak point of the "arctanh" approach. Anyhow, as we stressed before ${ }^{5}$ there is no exact theoretical basis for this procedure.

The present approach is quite different in this respect, the parameter $(\mathrm{H})$ required for straightening ("idealizing") the calibration curve is calculated directly from the curve itself. If in the application of the "parabolic" approach to the calculation of thrombin concentrations, the value of $\mathrm{H}$ found in the calibrator curve is sufficiently close to that in the TG curve cannot be determined directly. We therefore calculated the values of $\mathrm{H}$ for thrombin and the corresponding ones for $\alpha_{2} \mathrm{M}-\mathrm{T}$ at all substrate- and albumin concentrations (figure 6) and found them close enough to be interchangeable, especially at the higher albumin concentrations

This can be explained by assuming that the substrate binds reversibly to albumin, but thrombin is capable of cleaving the bound substrate whereas $\alpha_{2} \mathrm{M}-\mathrm{T}$ is not. This is corroborated by the fact that substrate that is irreversibly bound to albumin cannot be split by $\alpha_{2} \mathrm{M}-\mathrm{T}^{7}$. In that case the relation between apparent $\mathrm{Km}(\mathrm{Km}$ app $)$ and real $\mathrm{Km}\left(\mathrm{Km}_{\text {real }}\right)$ is given by $K m_{\text {app }}=K m_{\text {real }} .(K d+A) / K d$, where $\mathrm{Kd}$ is the dissociation constant between substrate and albumin and $\mathrm{A}$ the albumin concentration. From the data in figure 5 (left frame) we then find a $\mathrm{Km}_{\text {real }}$ of around $220 \mu \mathrm{M}$ and a $\mathrm{Kd}$ of around $30 \mathrm{mg} / \mathrm{ml}$.

These data cannot be quantitatively applied to the plasma situation because in plasma many ligands may compete for binding of albumin with the substrate and also other (lipo-)proteins may bind the substrate. If the situation in our experiments and in plasma is roughly comparable, however, we may expect that in the normal range $(35-50 \mathrm{mg} / \mathrm{ml}) 40-50 \%$ of the substrate is in free form. 


\section{References}

1. Hemker, H. C. et al. Calibrated Automated Thrombin Generation Measurement in Clotting Plasma. Pathophysiol. Haemost. Thromb. 33, 4-15 (2003).

2. Hemker, H. et al. The calibrated automated thrombogram (CAT): a universal routine test for hyper-and hypocoagulability. Pathophysiol. Haemost. Thromb. 32, 249253 (2002).

3. Arneson, W. \& Brickell, J. Clinical chemistry : a laboratory perspective. (F.A. Davis Co., 2007).

4. Peters, T. Serum Albumin. in Advances in Protein Chemistry (eds. Anfinsen, C. B., Edsall, J. T. \& Richards, F. M.) 37, 161-245 (Academic Press, 1985).

5. Hemker, H. C., Hemker, P. W., Al Dieri, R. \& others. The technique of measuring thrombin generation with fluorescent substrates: 4. The H-transform, a mathematical procedure to obtain thrombin concentrations without external calibration. Thromb. Haemost. 101, 171-177 (2009).

6. Eisenthal, R. \& Cornish-Bowden, A. The direct linear plot. A new graphical procedure for estimating enzyme kinetic parameters. Biochem. J. 139, 715-720 (1974).

7. Wagenvoord, R. J., Deinum, J., Elg, M. \& Hemker, H. C. The paradoxical stimulation by a reversible thrombin inhibitor of thrombin generation in plasma measured with thrombinography is caused by a2-macroglobulin-thrombin. J. Thromb. Haemost. 8, 12811289 (2010). 

Chapter 8

Correction of the Effect of the Fluorogenic Thrombin Substrate Used in Calibrated Automated Thrombography on Physiological Thrombin Generation.

Romy M.W. Kremers, Martijn Moorlag, Rob J. Wagenvoord, H. Coen Hemker, Bas de Laat. Manuscript in preparation 


\section{Abstract}

Background Calibrated automated thrombography (CAT) is a global haemostasis assay and its outcome correlates well with bleeding and thrombosis risk. Thrombin generation $(T G)$ is measured using the fluorogenic thrombin substrate ZGGR-AMC. The presence of any thrombin substrate causes a certain amount of thrombin to be substrate-bound, depending on the kinetics of the substrate. Therefore, every thrombin substrate will affect the process of TG itself.

Methods TG was measured with 100-600 $\mu$ M ZGGR-AMC in pooled normal plasma. Prothrombin conversion and thrombin inactivation were calculated. Computational modeling was used to predict the course of TG in the absence of ZGGR-AMC in pooled normal plasma and in 25 healthy subject TG curves.

Results ZGGR-AMC increases the ETP and peak height in a dose-dependent manner. Lag-time and time-to-peak are prolonged at increasing ZGGR-AMC concentration, and the same effect was observed when measuring clotting times. ZGGR-AMC does not affect prothrombin conversion. All effects seen on TG can be explained by the dose-dependent attenuation of inactivation. A computational model of thrombin inactivation was used to "filter out" the effect of ZGGR-AMC on TG. Subsequently, TG curves corrected for interference by ZGGR-AMC are significantly lower than experimental TG curves in healthy subjects.

Conclusions Fluorogenic substrate ZGGR-AMC interferes with physiological TG by attenuating only thrombin inactivation. Computational modelling was successfully used to predict physiological TG curves, i.e. in the absence of ZGGRAMC. TG experiments performed with lower substrate concentrations provide a more accurate representation of the physiological condition. 


\section{Introduction}

Thrombin is the key enzyme in coagulation and therefore the target of most global diagnostic tests ${ }^{1-3}$. In routine clinical diagnostic tests such as the prothrombin time (PT) and the activated partial thromboplastin time (aPTT), the time is measured until the first traces of thrombin and a fibrin clot are formed. However, the bulk amount of thrombin is generated after the initial clot formation and it is this parameter, the total amount of thrombin generation (endogenous thrombin potential or ETP) that has been shown to be predictive of the bleeding or thrombosis risk ${ }^{4-11}$. A clotting time measures only $1 \%$ of the total amount of thrombin formed and does not consider anticoagulant processes, such as the inhibition of thrombin by antithrombin or the function of the APC system ${ }^{4,12}$. Moreover, whereas clotting times are insensitive to the activity of some anticoagulants, such as heparin, all known anticoagulant medication, including the new oral anticoagulants, have been shown to decrease thrombin generation $(\mathrm{TG})^{4,5,13-19}$.

Over the last decades, the continuous measurement of thrombin generation (TG) in plasma by calibrated automated thrombinography (CAT) has become indispensable to thrombosis and haemostasis research ${ }^{4,5,10,12,13}$. A complication of the CAT method which is often mentioned, is that the fluorogenic substrate Z-GlyGly-Arg-7-amino-4-methylcoumarin (ZGGR-AMC), which is added to probe thrombin activity, affects the process of thrombin generation itself ${ }^{20-23}$. The presence of any thrombin substrate causes part of the thrombin to be substrate bound (depending on the value of $\mathrm{Km}$ ) and therefore less thrombin is available for interaction with its inhibitors antithrombin (AT) and $\alpha_{2}$ Macroglobulin $\left(\alpha_{2} M\right)$, or for the feedback activation of FV and FVIII.

It is often questioned to which extent these effects of the fluorogenic substrate ZGGR-AMC affect the TG curve. Therefore, we aim to quantify the effect of the ZGGR-AMC concentration on thrombin generation and fibrin clot formation. We have recently developed a technique to split a TG curve into its underlying processes: prothrombin conversion and thrombin inactivation ${ }^{24}$. The thrombin decay rate is predicted computationally, based on the AT, $\alpha_{2} \mathrm{M}$ and fibrinogen level of a concentration, and considers the ZGGR-AMC concentration. An advantage of this computational model is that the input variables ( $A T, \alpha_{2} M$, fibrinogen and ZGGR-AMC concentrations) can be varied to perform in silico experiments. In our case, we aim to predict the course of thrombin generation in the absence of ZGGRAMC. 


\section{Materials and methods}

\section{Sample collection and handling}

Blood was collected from healthy volunteers by venipuncture after informed consent in concordance with the declaration of Helsinki. Platelet poor plasma (PPP) was prepared by centrifuging twice at $2821 \cdot \mathrm{g}$ for 10 minutes, and stored as individual samples, or pooled and stored as normal pooled plasma (NPP) at $-80^{\circ} \mathrm{C}$.

\section{Materials}

ZGGR-AMC was purchased at Bachem (Bubendorf, Switzerland) and dissolved in DMSO. Calibrator ( $\alpha_{2} \mathrm{M}-\mathrm{T}$ complex) was prepared as described by Hemker et al. ${ }^{4}$. Innovin recombinant human Tissue Factor (rTF) was used (Dade-Behring, Marburg, Germany). Procoagulant phospholipids (PL), containing: 60\% dioleoyl PC, 20\% dioleoyl PS, and $20 \%$ dioleoyl PE, were prepared as described elsewhere ${ }^{25}$. Chromogenic thrombin substrate S2238 was synthesized in house. Unfractionated heparin and bovine serum albumin were purchased at Sigma-Aldrich (Saint Louis, Missouri, USA). Bovine thrombin was purified in house as described by Church $^{26}$ and bovine antithrombin according to the protocol of Thaler $^{27}$. Staphylocoagulase was purified in house as described by Hendrix et $\mathrm{al}^{28}$.

\section{Measurement of coagulation factor levels}

Functional AT levels were determined by titration with thrombin as previously described (intra-assay $C V=5 \%$ and inter-assay $C V=13 \%)^{24}$. Functional $\alpha_{2} M$ levels were determined as previously described (intra-assay CV $=3 \%$ and inter-assay CV = $9 \%)^{24}$. Prothrombin levels were measured in plasma and serum samples as previously described (intra-assay CV $=4 \%$ and inter-assay CV $=11 \%)^{24}$. Plasma fibrinogen levels were measured by the Clauss method ${ }^{29}$.

\section{Measurement of clotting times}

Clotting assays were performed using a START coagulometer (Diagnostica Stago, Gennevilliers, France). $100 \mu \mathrm{L}$ NPP was incubated with $25 \mu \mathrm{L}$ tissue factor ( 1 or 5 pM f.c.) and phospholipids ( $4 \mu \mathrm{M}$ f.c.) at $37^{\circ} \mathrm{C}$ for 3 minutes. Coagulation was triggered by the addition of $25 \mu \mathrm{L}$ trigger mix containing calcium ( $100 \mathrm{mM})$ and 0 , $600,1200,1800,2400,3000$ or $3600 \mu M$ ZGGR-AMC. After triggering, the clotting time was recorded.

\section{Thrombin generation in plasma}

Calibrated Automated Thrombography (CAT) was performed as previously described $^{4}$. All wells contained $80 \mu \mathrm{l}$ plasma and $20 \mu \mathrm{l}$ of tissue factor (TF; 1 or 5 pM f.c.) and phospholipids (PL; $4 \mu \mathrm{M}$ f.c.). To calibrator wells, $20 \mu \mathrm{l}$ of calibrator was added instead. NPP was spiked with fluorogenic substrates to obtain final concentrations of $100,200,300,400,500$ or $600 \mu \mathrm{M}$ ZGGR-AMC in the well. 
Thrombin generation was initiated by the addition of $20 \mu \mathrm{l} \mathrm{CaCl} 2(16.6 \mathrm{mM})$. The TG fluorescence data was converted to thrombin generation curves, as described elsewhere $^{30}$ and used to perform additional computational analysis to extract prothrombin conversion curves ${ }^{24}$.

\section{Measurement of thrombin inactivation in plasma}

Thrombin inactivation was quantified by adding a fixed amount of human thrombin $(500 \mathrm{nM}$ f.c.; $20 \mu \mathrm{l})$ to non-recalcified, preheated $\left(37^{\circ} \mathrm{C}\right)$ plasma. The course of the thrombin inactivation was monitored via the cleavage of fluorogenic thrombin substrates ZGGR-AMC (100-600 $\mu \mathrm{M}$ f.c.; $20 \mathrm{ul})$. The course of thrombin concentration in time was calculated from the fluorescence trace in the same way as thrombin generation curves $^{30}$.

\section{Calculation of prothrombin conversion and thrombin inactivation}

Thrombin decay was predicted by the previously described and validated computational model ${ }^{24}$. This model consists of a set of ordinary differential equations, which describe the rate of thrombin removal over time, based on the plasma $\mathrm{AT}, \alpha_{2} \mathrm{M}$ and fibrinogen levels and the current thrombin concentration. At any moment during the course of the thrombin generation process, the net velocity of thrombin formation $(\mathrm{d}(\mathrm{T}) / \mathrm{dt})$ is the result of the velocity of prothrombin conversion $-d(P) / d t)$ and thrombin decay by its inhibitors (d(T-inh)/dt) (Eq. 1). Therefore, if TG is measured in a sample and thrombin decay can be predicted, prothrombin conversion can be calculated (Eq. 2).

Eq. 1. $\frac{d(T)}{d t}=-\frac{d(P)}{d t}-\frac{d(T-i n h)}{d t}$

Eq. 2. $-\frac{d(P)}{d t}=\frac{d(T)}{d t}+k_{A T} \bullet[A T]_{t} \bullet\left[T_{\text {free }}\right]_{t}+k a_{2} M \bullet\left[a_{2} M\right]_{t} \bullet\left[T_{\text {free }}\right]_{t}$

The apparent values of $k_{A T}$ and $k_{a 2 M}$ are dependent on the fluorogenic substrate concentration, which are determined in the thrombin inactivation experiment described above. Therefore, the influence of the fluorogenic substrate on thrombin inactivation was previously included in the computational mode ${ }^{24}$. Thrombin binds to ZGGR-AMC in an equilibrium of $\mathrm{S} /(\mathrm{Km}+\mathrm{S})$, causing only the fraction $\mathrm{Km} /(\mathrm{Km}+\mathrm{S})$ of the total amount of thrombin to be available for interaction with its inhibitors (Eq. 3). The value of $\mathrm{Km}$ in plasma-like buffer was previously determined to be $358 \mu \mathrm{M}^{24}$.

Eq.3. $\left[T_{\text {free }}\right]_{t}=K m /\left(K m+S_{t}\right) \cdot[T]_{t}$ 


\section{In silico experimentation}

In the current work we show that ZGGR-AMC affects thrombin inactivation, but not prothrombin conversion (figures 2-3). Subsequently, prothrombin conversion curves calculated from TG curves measured at varying concentrations of fluorogenic substrate do not differ significantly with the exception that the lagtime is prolonged at increasing ZGGR-AMC concentrations. Therefore, it is possible to use the prothrombin conversion curve in combination with the predicted thrombin inactivation rate in the absence or presence of substrate to demonstrate the effect of fluorogenic substrates on thrombin generation in an in silico experiment. The lag-time in the absence of substrate was extrapolated from the data obtained at increasing ZGGR-AMC concentrations.

\section{Statistics}

The Statistical Package for the Social Sciences (SPSS) was used to determine the statistical significance of the results. The distribution of the data was tested with a Shapiro-Wilk test and the statistical significance of differences between ZGGR-AMC concentrations was determined by an ANOVA or Kruskal-Wallis analysis, accordingly.

\section{Results}

Thrombin generation was measured in pooled normal plasma containing increasing concentrations of the fluorogenic thrombin substrate ZGGR-AMC (figure 1A-B). The ETP and peak height at both 1 and $5 \mathrm{pM}$ TF were increased with increasing ZGGR-AMC concentration (figure $1 \mathrm{C}$-D). The lag-time and time-to-peak were significantly prolonged at increasing ZGGR-AMC concentrations, in pooled normal plasma triggered with $1 \mathrm{pM} \mathrm{TF}$, but not $5 \mathrm{pM}$ TF (figure 1E-F). The prolongation of the TG lag-time was reflected in a concentration-dependent prolongation of the clotting time in pooled normal plasma containing increasing concentrations of ZGGR-AMC (table 1).

Table 1. The effect of the ZGGR-AMC concentration on the tissue factor induced clotting time.

The mean ( \pm standard deviation) of 4 experiments is shown.

\begin{tabular}{|l|l|l|l|l|l|l|l|l|}
\hline $\begin{array}{l}\text { [ZGGR-AMC] } \\
(\mu \mathrm{M})\end{array}$ & 0 & $\begin{array}{l}10 \\
0\end{array}$ & $\begin{array}{l}20 \\
0\end{array}$ & $\begin{array}{l}30 \\
0\end{array}$ & $\begin{array}{l}40 \\
0\end{array}$ & $\begin{array}{l}50 \\
0\end{array}$ & $\begin{array}{l}60 \\
0\end{array}$ & $\begin{array}{l}\text { p-value } \\
\text { (ANOVA) }\end{array}$ \\
\hline $\begin{array}{l}\text { Clotting time } \\
\text { at 1 pM TF }\end{array}$ & $\begin{array}{l}123 \\
\pm 9\end{array}$ & $\begin{array}{l}126 \\
\pm 4\end{array}$ & $\begin{array}{l}131 \\
\pm 8\end{array}$ & $\begin{array}{l}131 \\
\pm 3\end{array}$ & $\begin{array}{l}132 \\
\pm 3\end{array}$ & $\begin{array}{l}130 \\
\pm 1\end{array}$ & $\begin{array}{l}142 \\
\pm 2\end{array}$ & 0.027 \\
\hline $\begin{array}{l}\text { Clotting time } \\
\text { at 5 pM TF }\end{array}$ & \pm 0 & 60 & 65 & 62 & 66 & 65 & 70 & 0.003 \\
(sec) & \pm 3 & \pm 3 & \pm 3 & \pm 2 & \pm 1 & \pm 1 & \\
\hline
\end{tabular}


Chapter 8 

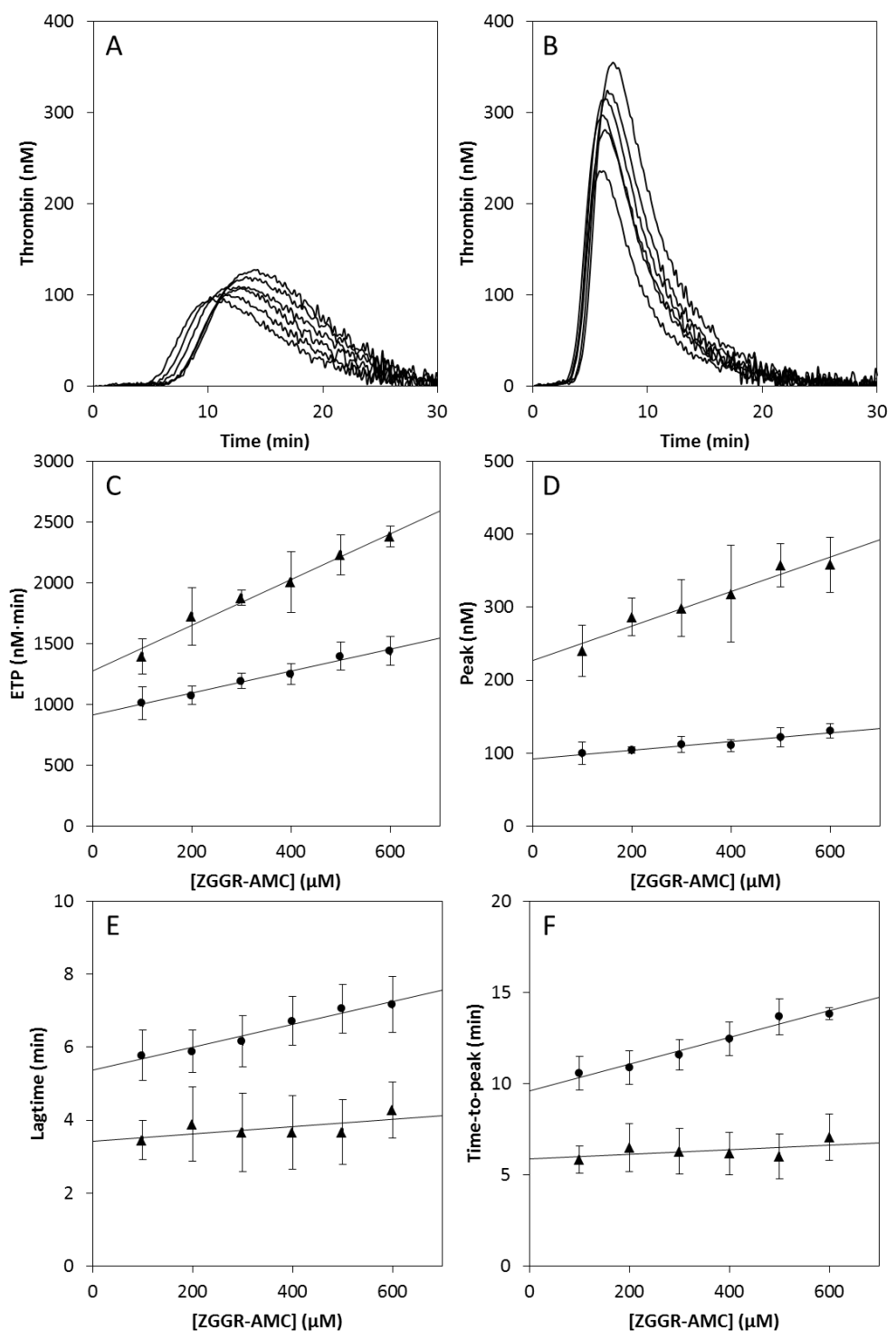

Figure 1. The effect of the fluorogenic substrate concentration on thrombin generation. Thrombin generation was measured at 1 pM TF (A) and 5 pM TF (B) in pooled normal plasma containing ZGGR-AMC (from bottom to top 100, 200, 300, 400, 500 and $600 \mu \mathrm{M}$ ). (C-D) ZGGR-AMC increases the ETP and peak in a concentration-dependent manner at 1 $\mathrm{pM}$ TF (circles, $\mathrm{R}=0.88, \mathrm{p}<0.001$ and $\mathrm{R}=0.73, \mathrm{p}=0.001$ ), and at $5 \mathrm{pM}$ TF (triangles, $\mathrm{R}=0.77$, $p<0.001$ and $R=0.67, p<0.001)$. ( $E-F)$ The lag-time and time-to-peak are prolonged at increasing concentrations of ZGGR-AMC, if TG is triggered with $1 \mathrm{pM}$ TF (circles, $R=0.70$, $p=0.002$ and $R=0.78, p<0.001$ ), but not with 5 pM TF (triangles). Symbols indicate that the mean of 5 experiments and the bars indicate the standard deviation. 
The thrombin generation curve is the combined result of the processes of prothrombin conversion and thrombin inactivation. To further investigate the effect of the fluorogenic substrates on TG, we determined their effect on prothrombin conversion and thrombin inactivation separately. Thrombin inactivation was measured in pooled normal plasma containing increasing concentrations of ZGGR-AMC (figure 2). ZGGR-AMC attenuated thrombin inactivation significantly in a concentration dependent manner. Thrombin decay by antithrombin and $\alpha_{2} \mathrm{M}$, the two most important thrombin inhibitors, were affected in a similar manner, as the apparent rate constant for thrombin decay for each inhibitor decreased at increasing ZGGR-AMC concentrations (figure 2B-C).
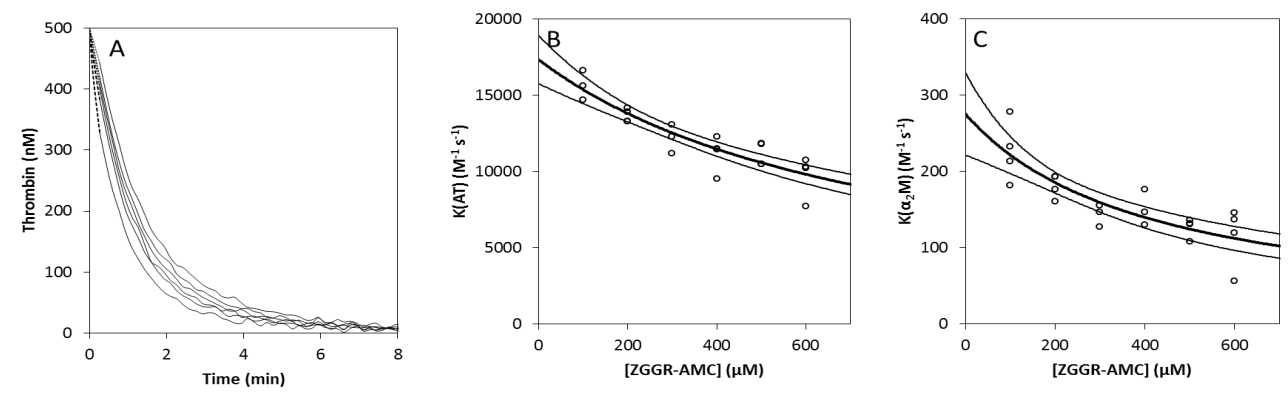

Figure 2. The effect of fluorogenic substrate concentration on the thrombin inactivation rate.

(A) The inactivation of $500 \mathrm{nM}$ thrombin was measured in non-recalcified pooled normal plasma containing 100, 200, 300, 400, 500, and $600 \mu \mathrm{M}$ ZGGR-AMC (bottom to top). (B-C) The apparent rate constant of thrombin decay by antithrombin $\left(k_{A T}\right)$ and by $\alpha_{2} M\left(k_{\alpha 2 M}\right)$ are inversely related to the ZGGR-AMC concentration $(p<0.001$ and $p<0.001)$. The data points were fitted by the formula $k_{\text {dec.experimental }}=k_{\text {dec.real }} /(1+[Z G G R-A M C] / K m)$, and the optimal fit and $95 \%$ confidence interval is shown. The value of $\mathrm{Km}$ was dependent on the inhibitor: $782 \mu \mathrm{M}$ for thrombin-AT formation and $410 \mu \mathrm{M}$ for $\alpha_{2} \mathrm{M}$-thrombin $\left(\alpha_{2} \mathrm{M}-\mathrm{T}\right)$ formation.

The apparent thrombin decay constants obtained in figure 2 were used to calculate the course of prothrombin conversion, based on the concept that the thrombin generation curve is defined by the processes of prothrombin conversion and thrombin inactivation. Therefore, if the (apparent) rate of thrombin inactivation and the thrombin generation curve itself are known, the course of prothrombin conversion can be calculated. Figure 3A-B show that ZGGR-AMC does not significantly affect the course of prothrombin conversion, except the prolongation of the lag-time at increasing ZGGR-AMC concentrations. Indeed, the total amount of prothrombin converted is not affected by increasing concentrations of ZGGR-AMC (figure $3 \mathrm{C}$ ), and the maximum rate of prothrombin conversion, remains unchanged at increasing ZGGR-AMC concentrations (figure 3D). 

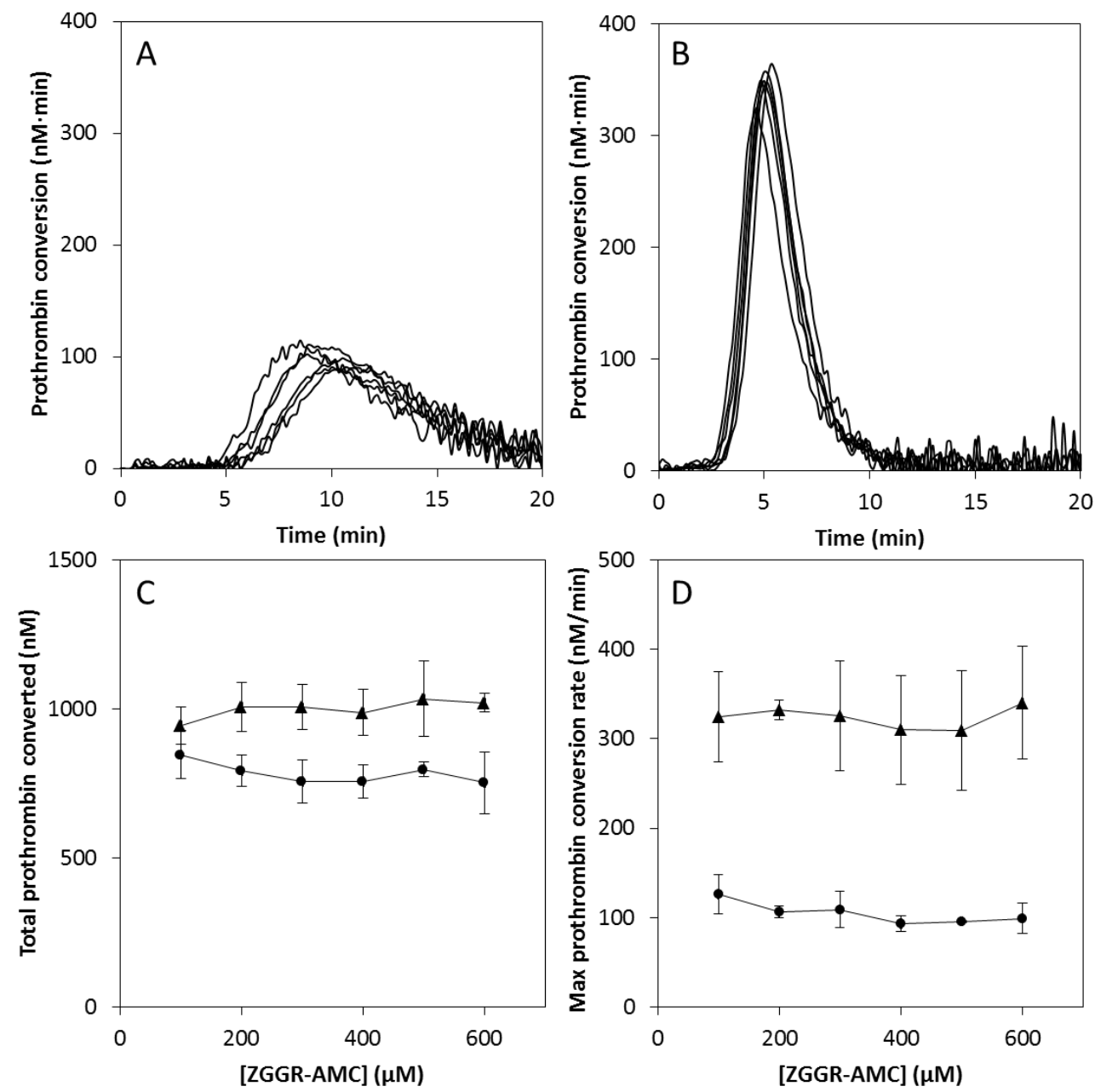

Figure 3. The effect of fluorogenic substrate concentration on the course of prothrombin conversion. Prothrombin conversion curves were calculated from TG curves measured at 1 pM TF (A) and 5 pM TF (B), in pooled normal plasma containing 100, 200, 300, 400, 500, and $600 \mu \mathrm{M}$ ZGGR-AMC (from left to right). (C) The total amount of prothrombin converted at increasing ZGGR-AMC concentrations remained constant at $1 \mathrm{pM}$ TF (circles) and $5 \mathrm{pM}$ TF (triangles). (D) The maximum rate of prothrombin conversion was unchanged at varying substrate concentration during TG indicated with 1 pM TF (circles) and 5 pM TF (triangles). Symbols indicate that the mean of 5 experiments and the bars indicate the standard deviation.

The calculated effects of ZGGR-AMC on prothrombin conversion were verified by in vitro experiments. The effect of the ZGGR-AMC concentration on the total amount of converted prothrombin in plasma was determined experimentally by 
measuring the prothrombin levels of each sample before and after thrombin generation. The data from table 2 show that ZGGR-AMC does not affect the total amount of prothrombin converted during TG triggered with either 1 or 5 pM TF. In addition, the total amount of thrombin-antithrombin (T-AT) and $\alpha_{2} \mathrm{M}-\mathrm{T}$ complexes formed during TG remains constant at all ZGGR-AMC concentrations (data not shown).

Table 2. The effect of the ZGGR-AMC concentration on the experimentally determined amount of prothrombin converted during thrombin generation. The mean ( \pm standard deviation) of 5 experiments is shown.

\begin{tabular}{|c|c|c|c|c|c|c|c|c|}
\hline $\begin{array}{l}\text { [ZGGR- } \\
\text { AMC] }(\mu M)\end{array}$ & 0 & 100 & 200 & 300 & 400 & 500 & 600 & $\begin{array}{l}\text { p-value } \\
\text { (ANOVA }\end{array}$ \\
\hline $\begin{array}{l}\text { Total } \\
\text { prothrombi } \\
\mathrm{n} \text { converted } \\
\text { at } 1 \mathrm{pM} \text { TF } \\
(\mu \mathrm{M})\end{array}$ & $\begin{array}{l}0.91 \\
\pm 0.03\end{array}$ & $\begin{array}{l}0.88 \\
\pm 0.03\end{array}$ & $\begin{array}{l}0.87 \\
\pm 0.03\end{array}$ & $\begin{array}{l}0.88 \\
\pm 0.03\end{array}$ & $\begin{array}{l}0.86 \\
\pm 0.05\end{array}$ & $\begin{array}{l}0.85 \\
\pm 0.06\end{array}$ & $\begin{array}{l}0.87 \\
\pm 0.04\end{array}$ & 0.676 \\
\hline $\begin{array}{l}\text { Total } \\
\text { prothrombi } \\
\mathrm{n} \text { converted } \\
\text { at } 5 \mathrm{pM} \text { TF } \\
(\mu \mathrm{M})\end{array}$ & $\begin{array}{l}1.03 \\
\pm 0.03\end{array}$ & $\begin{array}{l}0.99 \\
\pm 0.04\end{array}$ & $\begin{array}{l}1.04 \\
\pm 0.04\end{array}$ & $\begin{array}{l}1.01 \\
\pm 0.03\end{array}$ & $\begin{array}{l}1.03 \\
\pm 0.04\end{array}$ & $\begin{array}{l}1.03 \\
\pm 0.03\end{array}$ & $\begin{array}{l}1.03 \\
\pm 0.03\end{array}$ & 0.530 \\
\hline
\end{tabular}

In silico experimentation was used to show what TG would look like if ZGGR-AMC would not be present in the plasma. Figure 4A-B shows the predicted TG curves triggered with 1 and $5 \mathrm{pM}$ TF in plasma not containing ZGGR-AMC and with 417 $\mu M$ ZGGR-AMC. 

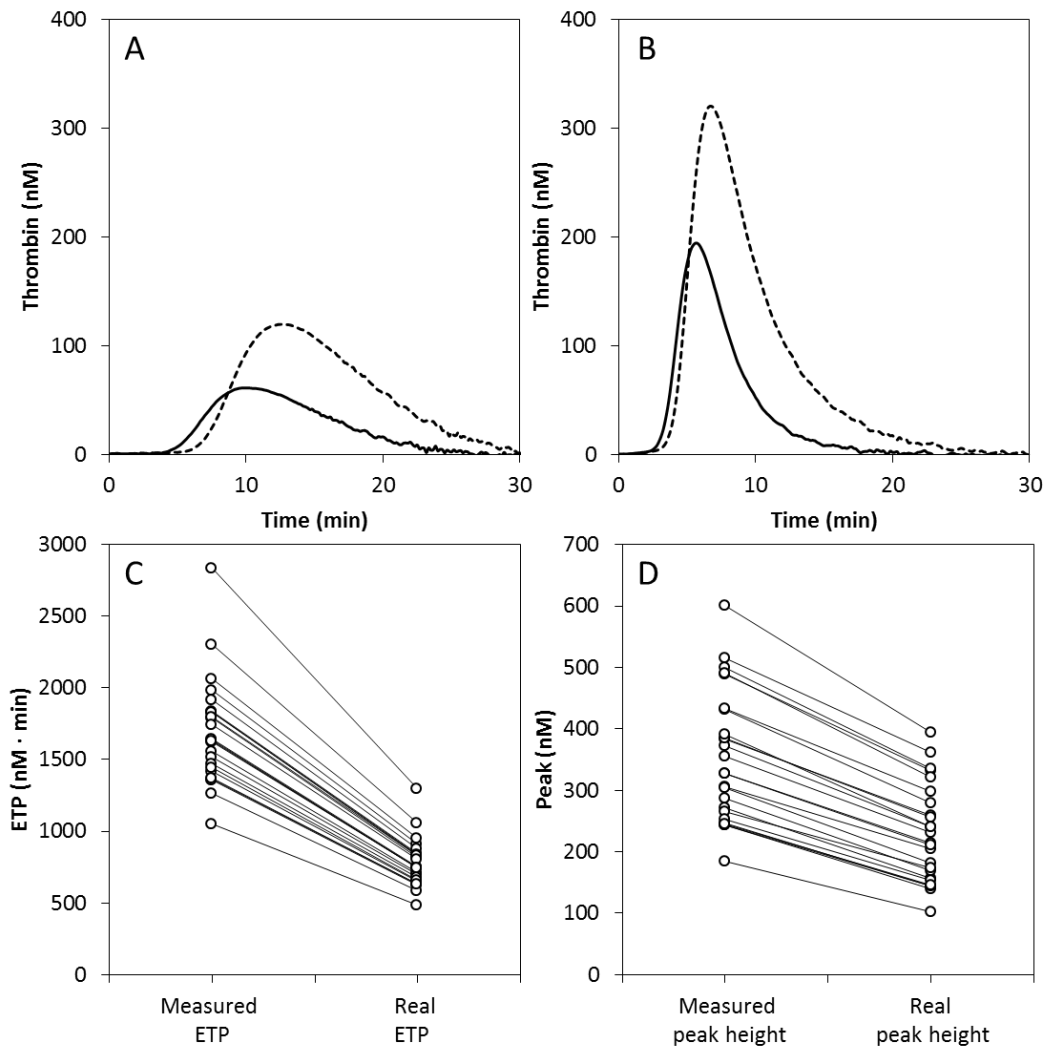

Figure 4. Computational simulation of thrombin generation in the absence of fluorogenic substrate.

Thrombin generation at 1 (A) and 5 (B) pM TF was predicted in silico in the absence (continuous line) and presence of $417 \mu \mathrm{M}$ of substrate (dashed line). (C-D) Thrombin generation in 25 healthy controls was measured using $417 \mu \mathrm{M}$ ZGGR-AMC. In silico experimentation was used to obtain each individual curve in the absence of ZGGR AMC and the measured ETP (C) and peak height (D) were compared to the 'real' ETP and peak height that would have been found in the samples if no ZGGR-AMC would have been present. $* * p<0.01$.

In the simulation, the average prothrombin conversion curve was used as a basis for the prediction of $T G$, because we have shown that the prothrombin conversion curve is not significantly affected by the ZGGR-AMC concentration. The effect of the ZGGR-AMC concentration on thrombin inactivation was predicted using the described computational model and the TG curve was calculated by adding the predicted thrombin inactivation rates up to the prothrombin conversion curve. The 
shortening of the lag-times was predicted by linear extrapolation from the data presented in figure 1E. Figure 4 shows that the real ETP is $46 \%$ of the ETP measured in the presence of $417 \mu \mathrm{M}$ ZGGR-AMC, which is the regular amount of fluorogenic substrate used in the CAT method, irrespective of the TF concentration. The real peak height is $51 \%$ and $61 \%$ of the measured peak height at 1 and 5 pM TF respectively (table 3 ). In addition, ZGGR-AMC prolongs the lagtime and time to peak. Using computational simulation, the ETP and the peak height were determined in the presence of $417 \mu \mathrm{M}$ ZGGR-AMC (measured value) or in the absence of ZGGR-AMC (real value) in 25 healthy subjects. The real ETP was $45 \pm 0 \%$ of the ETP measured at $417 \mu \mathrm{M}$ ZGGR-AMC and the real peak height was $63 \pm 4 \%$ of the measured peak height.

Table 3. In silico prediction of thrombin generation parameters in the absence and presence of $417 \mu M Z G G R-A M C$.

\begin{tabular}{|c|c|c|c|c|}
\hline & \multicolumn{2}{|l|}{$1 \mathrm{pM} \mathrm{TF}$} & \multicolumn{2}{|l|}{$5 \mathrm{pM} \mathrm{TF}$} \\
\hline & $\begin{array}{l}\text { No ZGGR- } \\
\text { AMC }\end{array}$ & $\begin{array}{l}417 \mu \mathrm{M} \text { ZGGR- } \\
\mathrm{AMC}\end{array}$ & $\begin{array}{ll}\text { No } & \text { ZGGR- } \\
\text { AMC } & \\
\end{array}$ & $\begin{array}{l}417 \mu \mathrm{M} \text { ZGGR- } \\
\mathrm{AMC}\end{array}$ \\
\hline $\begin{array}{ll}\text { ETP } & \text { (nM } \\
\min ) & \\
\end{array}$ & 647 & 1392 & 987 & 2119 \\
\hline Peak (nM) & 61 & 120 & 195 & 320 \\
\hline $\begin{array}{l}\text { Lag-time } \\
(\min )\end{array}$ & 5.38 & 6.67 & 3.14 & 3.47 \\
\hline $\begin{array}{l}\text { Time-to- } \\
\text { peak (min) }\end{array}$ & 10.00 & 12.83 & 5.67 & 6.67 \\
\hline
\end{tabular}

\section{Discussion}

We show that the fluorogenic substrate ZGGR-AMC, which is used in the CAT method, increases ETP and peak height in a dose-dependent manner, and prolongs the lag-time and time-to-peak. The effect of the ZGGR-AMC concentration on TG in a plasma system is comparable to its effect on TG in a buffer system, as previously shown by Butenas et al. ${ }^{21}$. The presence of an exogenous thrombin substrate is known to attenuate all thrombin catalysed reactions, because the binding of thrombin to the fluorogenic substrate decreases the amount of thrombin that is available for its physiological reactions. Ideally, the fluorogenic thrombin substrate should have a large $\mathrm{Km}$, causing only a small fraction of the total amount of thrombin to be substrate bound and therewith minimizing the effect. In practice, however, the ZGGR-AMC substrate has a Km of $358 \mu \mathrm{M}$, which is approximately the concentration at which it is generally used in the CAT method $(417 \mu \mathrm{M})^{31}$. Consequently, $54 \%$ of all thrombin will be bound to the fluorogenic substrate during the process of thrombin generation, if substrate consumption is not 
considered. Subsequently, the presence of ZGGR-AMC has a substantial dosedependent effect on the course of thrombin generation.

Thrombin generation is the net result of thrombin production (prothrombin conversion) and thrombin inactivation ${ }^{24}$. During $T G$, thrombin stimulates its own generation via feedback activation of FV and FVIII, ${ }^{52-35}$, which results in a burst of thrombin production. The generated thrombin is inactivated by the plasmatic thrombin inhibitors antithrombin and $\alpha_{2} \mathrm{M}^{36-38}$. We show that the feedback reactions are affected by the presence of ZGGR-AMC, as the lag-time and clotting time increase with increasing ZGGR-AMC concentrations. The effect of ZGGR-AMC on procoagulant processes, however, is confined to the initial phase of thrombin generation, as the amount of prothrombin converted during TG is not influenced significantly by the plasma ZGGR-AMC concentration (table 2).

The main effect of the ZGGR-AMC concentration on thrombin generation is its attenuation of thrombin inactivation. The reaction of thrombin with antithrombin and $\alpha_{2} \mathrm{M}$ are attenuated in a dose-dependent manner by the ZGGR-AMC concentration, as both the inhibitors and ZGGR-AMC compete for the active site of thrombin. The reduced inhibition rate prolongs the half-life of thrombin in the clotting plasma and causes the ETP to increase up to $215 \%$ and the peak height up to $165 \%$ (5 pM TF trigger).

Using a recently developed computational model for thrombin inactivation, it is possible to simulate the effect of ZGGR-AMC concentration on thrombin generation. This model was used to predict the effect of the ZGGR-AMC concentration on thrombin generation in a group of 25 healthy controls. The effect of ZGGR-AMC presence during TG is remarkably comparable within a group of healthy subjects. The in silico predicted ETP in the absence of ZGGR-AMC was $46 \%$ of the ETP measured at $417 \mu \mathrm{M}$ ZGGR-AMC, irrespective of the TF concentration used. The ratio of the peak height in the absence and presence of ZGGR-AMC is quite stable in healthy controls, although the percentage differs between the conditions depending on the TF concentration used to trigger TG. Therefore, it is not possible to predict the 'real' peak height by a simple rule of thumb, but computational modelling can provide a solution for this problem. The current model can be used to convert all TG curves measured using ZGGR-AMC to a predicted 'real' TG curve.

Finally, we must consider the possibility of simply lowering the substrate concentration to prevent undue interaction of the substrate with the physiological process. As the substrate concentration becomes lower the life span of thrombin decreases and this makes that less substrate is consumed. Therefore, it is to be expected that at lower substrate concentrations the substrate will not run out. This 
idea is supported by the experiments in which a concentration range of ZGGR-AMC substrate was used to measure TG, and no signs of substrate depletion were observed at initial concentrations of $>100 \mu \mathrm{M}$ (see above). 


\section{References}

1. Bates, S. M. \& Weitz, J. I. Coagulation Assays. Circulation 112, e53-e60 (2005).

2. Hirsh, J. Oral Anticoagulant Drugs. N. Engl. J. Med. 324, 1865-1875 (1991).

3. Lange, U., Nowak, G. \& Bucha, E. Ecarin Chromogenic Assay - A New Method for Quantitative Determination of Direct Thrombin Inhibitors Like Hirudin. Pathophysiol. Haemost. Thromb. 33, 184-191 (2003).

4. Hemker, H. et al. The calibrated automated thrombogram (CAT): a universal routine test for hyper-and hypocoagulability. Pathophysiol. Haemost. Thromb. 32, 249-253 (2002).

5. Al Dieri, R., de Laat, B. \& Hemker, H. C. Thrombin generation: What have we learned? Blood Rev. 26, 197-203 (2012).

6. van Hylckama Vlieg, A. et al. Elevated endogenous thrombin potential is associated with an increased risk of a first deep venous thrombosis but not with the risk of recurrence. Br. J. Haematol. 138, 769-774 (2007).

7. Ay, C. et al. Prediction of venous thromboembolism in patients with cancer by measuring thrombin generation: results from the Vienna Cancer and Thrombosis Study. J. Clin. Oncol. Off. J. Am. Soc. Clin. Oncol. 29, 2099-2103 (2011).

8. Wielders, S. et al. The Routine Determination of the Endogenous Thrombin Potential, First Results in Different Forms of Hyper- and Hypocoagulability. Thromb. Haemost. 77, 629-636 (1997).

9. Beltrán-Miranda, C. P., Khan, A., Jaloma-Cruz, A. R. \& Laffan, M. A. Thrombin generation and phenotypic correlation in haemophilia A. Haemophilia 11, 326-334 (2005).

10. Ten Cate, H. Thrombin generation in clinical conditions. Thromb. Res. 129, 367-370 (2012).

11. Gerotziafas, G. T. et al. Towards a standardization of thrombin generation assessment: The influence of tissue factor, platelets and phospholipids concentration on the normal values of Thrombogram-Thrombinoscope assay. Thromb. J. 3, 16 (2005). 12. Dargaud, Y., Trzeciak, M. C., Bordet, J. C., Ninet, J. \& Negrier, C. Use of calibrated automated thrombinography \pm thrombomodulin to recognise the prothrombotic phenotype. Thromb. Haemost. 96, 562-567 (2006).

13. Hemker, H. C., Hemker, P. W., Al Dieri, R. \& others. The technique of measuring thrombin generation with fluorescent substrates: 4 . The H-transform, a mathematical procedure to obtain thrombin concentrations without external calibration. Thromb. Haemost. 101, 171-177 (2009).

14. Tripodi, A. The Long-Awaited Whole-Blood Thrombin Generation Test. Clin. Chem. 58, 1173-1175 (2012).

15. Castoldi, E. \& Rosing, J. Thrombin generation tests. Thromb. Res. 127, Supplement 3, S21-S25 (2011).

16. Bloemen, S., Hemker, H. C. \& Al Dieri, R. Large inter-individual variation of the pharmacodynamic effect of anticoagulant drugs on thrombin generation. Haematologica 98, 549-554 (2013). 
17. Herrmann, R. et al. Thrombin generation using the calibrated automated thrombinoscope to assess reversibility of dabigatran and rivaroxaban. Thromb. Haemost. 112, 989-995 (2014).

18. Serebruany, V. et al. Effects of dabigatran in vitro on thrombin biomarkers by Calibrated Automated Thrombography in patients after ischemic stroke. J. Thromb. Thrombolysis 33, 22-27 (2012).

19. Arachchillage, D. R. J. et al. Rivaroxaban and warfarin achieve effective anticoagulation, as assessed by inhibition of TG and in-vivo markers of coagulation activation, in patients with venous thromboembolism. Thromb. Res. 135, 388-393 (2015).

20. Mann, K. G. Is there value in kinetic modeling of thrombin generation: Yes. J. Thromb. Haemost. JTH 10, 1463-1469 (2012).

21. Butenas, S. \& Mann, K. G. Caution in the interpretation of continuous thrombin generation assays. J. Thromb. Haemost. 5, 1084-1085 (2007).

22. Hemker, H. C., Wielders, S., Kessels, H. \& Béguin, S. Continuous registration of thrombin generation in plasma, its use for the determination of the thrombin potential. Thromb. Haemost. 70, 617-624 (1993).

23. Brummel, K. E., Paradis, S. G., Butenas, S. \& Mann, K. G. Thrombin functions during tissue factor-induced blood coagulation. Blood 100, 148-152 (2002).

24. Kremers, R. M. W., Peters, T. C., Wagenvoord, R. J. \& Hemker, H. C. The balance of pro- and anticoagulant processes underlying thrombin generation. J. Thromb. Haemost. 13, 437-447 (2015).

25. Rosing, J., Tans, G., Govers-Riemslag, J., Zwaal, R. \& Hemker, H. C. The role of phospholipids and factor $V a$ in the prothrombinase complex. J. Biol. Chem. 255, 274283 (1980).

26. Church, F. C. \& Whinna, H. C. Rapid sulfopropyl-disk chromatographic purification of bovine and human thrombin. Anal. Biochem. 157, 77-83 (1986).

27. Thaler, E. \& Schmer, G. A Simple Two-step Isolation Procedure for Human and Bovine Antithrombin II/III (Heparin Cofactor): a Comparison of Two Methods. Br. J. Haematol. 31, 233-243 (1975).

28. Hendrix, H., Lindhout, T., Mertens, K., Engels, W. \& Hemker, H. C. Activation of human prothrombin by stoichiometric levels of staphylocoagulase. J. Biol. Chem. 258, 3637-3644 (1983).

29. Clauss, A. [Rapid physiological coagulation method in determination of fibrinogen]. Acta Haematol. 17, 237-246 (1957).

30. Hemker, H. C. \& Kremers, R. Data management in Thrombin Generation. Thromb. Res. 131, 3-11 (2013).

31. Hemker, H. C. \& Béguin, S. Thrombin generation in plasma: its assessment via the endogenous thrombin potential. Thromb. Haemost. 74, 134-138 (1995).

32. Spronk, H. M. H., Govers-Riemslag, J. W. P. \& ten Cate, H. The blood coagulation system as a molecular machine. BioEssays News Rev. Mol. Cell. Dev. Biol. 25, 1220-1228 (2003).

33. Versteeg, H. H., Heemskerk, J. W., Levi, M. \& Reitsma, P. H. New fundamentals in hemostasis. Physiol. Rev. 93, 327-358 (2013). 
34. Butenas, S., Branda, R. F., van't Veer, C., Cawthern, K. M. \& Mann, K. G. Platelets and phospholipids in tissue factor-initiated thrombin generation. Thromb. Haemost. 86, 660-667 (2001).

35. Davie, E. W., Fujikawa, K. \& Kisiel, W. The coagulation cascade: initiation, maintenance, and regulation. Biochemistry 30, 10363-10370 (1991).

36. Djie, M. Z., Stone, S. R. \& Bonniec, B. F. L. Intrinsic Specificity of the Reactive Site Loop of a1-Antitrypsin, a1-Antichymotrypsin, Antithrombin III, and Protease Nexin I. J. Biol. Chem. 272, 16268-16273 (1997).

37. Jesty, J. The kinetics of inhibition of alpha-thrombin in human plasma. J. Biol. Chem. 261, 10313-10318 (1986).

38. Beguin, S., Kessels, H., Dol, F. \& Hemker, H. The consumption of antithrombin III during coagulation, its consequences for the calculation of prothrombinase activity and the standardisation of heparin activity. Thromb. Haemost. 68, 136-142 (1992). 


Chapter 9

General Discussion and Conclusions 
The global hemostatic assay that measures thrombin generation (TG) in plasma increasingly gained attention as a diagnostic tool in the field of thrombosis and hemostasis. The Calibrated Automated Thrombin Generation test (CAT-TG) ${ }^{1}$ has been used in research laboratories for over 15 years now. And has over this period of time been implemented in over a thousand research laboratories worldwide.

Throughout the last decades considerable evidence has been published that the amount of thrombin activity measured with $\mathrm{TG}$ is an indicator of the risk of bleeding $^{2-5}$ as well as venous thrombosis ${ }^{6-14}$. TG can be used to distinguish mild from severe bleeding phenotypes in hemophilia A patients with comparable factor VIII levels ${ }^{15}$. It also relates to the risk of arterial thrombosis, be it in a less straightforward manner ${ }^{16-18}$. It is a common denominator of the effect of all anticoagulants ${ }^{19-26}$ and, when it is measured in platelet rich plasma, it is inhibited by platelet inhibitors ${ }^{27,28}$. Therefore, it has a promising outlook for use as a clinical parameter. Nevertheless, its implementation in the clinical laboratory until this moment is very limited.

One reason is that the CAT-TG is not a fully automated method like most other methods in the clinical routine laboratory. It still requires an amount of oldfashioned laboratory labour. Apart from the fact that this is not in line with modern laboratory management, it also introduces the executor as a cause of inter-laboratory variability. Strict training of the technicians is a sine qua non for obtaining acceptable results (Prof. Y. Dargaud, personal communication).

Another way to introduce TG in the clinic is by so called near patient testing or point of care (POC) testing ${ }^{29-31}$. In chapter 2 we described the development of a near patient, miniaturized device based upon a previously published method, developed in our laboratories, that allows the measurement of TG in wholeblood $^{32}$. Measurement in whole blood is necessary anyhow because obtaining plasma by centrifugation is hard to realise in a point of care situation. Anyhow, a full TG-curve requires about 15 minutes, which is long if an immediate answer is required as e.g. in emergency bleedings.

To further decrease the turnover time an alternative calculation method is presented in which only the data until the peak of the TG are used. Although much information present in the total TG is thus discarded, it can be argued that both pro- and antithrombotic processes contribute to the peak value and the area under the TG curve up to the peak value. In how far this information is useful and sufficient remains to be determined for each individual pathological condition. One can imagine e.g. that it suffices as a quick estimate of the effects of vitamin $\mathrm{K}$ antagonists and heparin but that it will do worse for direct inhibitors of factor $\mathrm{Xa}$, due to the blunted form of the TG-peak that such anticoagulants induce. 
In chapter 2 we show that we have been successfully able to miniaturize the device to have a similar inter-assay variation as the original test. The fact that only one sample can be measured at any time excludes determination of the intra-assay variation. Also, the impossibility of a simultaneous control step will increase the chances of introducing errors. Nevertheless, a comparison of the results obtained with the adapted calculation method to the standard shows decent correlation coefficients $0.868,0.998,0.993$ and 0.981 for the (peak)ETP, peak, lag-time and time-to-peak, respectively. The imperfect correlations are most likely due to the fact that the information from the decay phase of thrombin generation is discarded.

Clinical validation of this test has been performed on a group of 33 patients that underwent cardiothoracic surgery, with the aim to check whether the test would predict the degree of blood loss. After division of the patients into two groups (severe and mild bleeders) based on the mean drainage volume, a statistically significant difference was found between the two groups for the peak-ETP and the peak. This does not proof that the test can be used for predictive purposes but it is indicative of a statistically significant association. In the designing of this experiment, we had to trade of between the desire to have a homogenous sample population and a large number of samples. We have chosen for the first and restricted the type of intervention to Coronary Artery Bypass Grafting (CABG). This caused the sample size to be small and leaves room for more extensive studies in the future.

Simultaneously with the development of the near patient TG device, we wanted to make use of the miniaturised set-up to explore possibilities of alternative applications of TG. The zebrafish being a more and more used model, notably in fundamental studies on the relation between the genome and anatomy and physiology, we wanted to investigate whether this animal also could be used in experiments on coagulation physiology.

In chapter 3 we show that by applying the miniaturised model to zebrafish we are able to generate TG curves with an acceptable inter-individual variation with a mean CV of $14 \%$. Moreover, we were able to detect a dose-response effect of two anticoagulant drugs: Rivaroxaban (a direct FXa inhibitor) and Melagatran (a direct Flla inhibitor). Despite an encouraging start, the lack of physiological similarity between zebrafish and humans has led us to decide not to continue this path any further at this moment. Nevertheless, we believe that being able to measure extremely small quantities of blood in such frail animals brings many opportunities for future animal studies. 
After this brief animal excursion, we understood that the miniaturized version, that we derived from the whole blood-TG method was in its current form not the way to quick clinical application.

We realized that one of the major drawbacks in the method remained the, relatively large, inter-laboratory variability and bad standardization criteria ${ }^{33-36}$ which are only partly ameliorated by normalization ${ }^{33-35,37}$. Notably in the case of (extreme) hypo coagulability normalization is not able to reduce the interlaboratory variation. As the matter of fact normalization actually caused an increase in variation of some of the parameters ${ }^{33}$.

We were in the lucky circumstance that, through the cooperation with a number of clinical labs, we could lay our hands on the raw fluorescence curves from a reproducibility study carried out in France. Analysis of these curves allowed us to gain insight into the reasons for the large interlaboratory variability (Chapter 4). The biggest contributor to the total error appeared to be the comparison between the TG-sample and the calibrator. We were able to assess the contribution of the calibrator to the total error by making use of the ratio between the ETP and the end-level of $\alpha_{2} \mathrm{M}-\mathrm{T}$. This can be done because the proportion, to which ETP is overor under-estimated by the calibration procedure, is the same as the proportion to which the final level of $\alpha_{2} \mathrm{M}-\mathrm{T}$ is under- or overestimated.

By applying this correction, the inter-laboratory CV for the ETP could be reduced from $16,3 \%$ to $11,7 \%$. A similar trend is observed for the thrombin peak in which the inter-laboratory CV is reduced from $17,4 \%$ to $11,9 \%$. It thus appears that the calibration step, that has been designed as a tool for quantification and elimination of the known (inner filter, substrate consumption and plasma colour) and unknown measurement artefacts, in fact contributes significantly to the experimental error. This phenomenon can, hypothetically, be attributed to two reasons: The first is that in any procedure in which the data from two observations (calibrator and TG-curve) are combined, the experimental error in the outcome sums-up. The second being that the calculations used to combine the outcomes introduce systematic errors.

As a second major source of error we could identify the inconsistency of temperature, in particular as a result of inadequate pre-heating of the sample. We therefore had a closer look into both temperature control and calculation methods. In chapter 4 we re-introduce the "diagnostic plot" as a tool to determine temperature stability at the start of the experiment. The diagnostic plot is a plot of the first derivative of the fluorescence curve against the value of the fluorescence itself ${ }^{38,39}$.

In a calibrator experiment performed at constant temperature, $\mathrm{dF} / \mathrm{dt}$ is at its maximum at zero time because both the substrate concentration is highest and 
the inner filter effect is still absent. An increase of $\mathrm{dF} / \mathrm{dt}$ at the start of the experiment can therefore only relate to an increase of temperature. The characteristic "hockey stick" shape of the first derivative of the calibrator curve can only indicate a rise in temperature at the start of the experiment as there is no other explanation for the increase in reaction velocity. This error is mainly due to the operator that has not pre-heated the sample to the exact value of that in the fluorometer. Design changes to the software that enforce a fixed pre-heating time as well as creating operator awareness of the importance of temperature could partly improve the situation, be it that the time required to attain the right temperature is longer than ten minutes and that evaporation of the sample begins to play a role then.

After having seen the important role of temperature in the CAT-TG and not having been able to find any conclusive literature on the topic of temperature and TG, we decided to investigate the influence of temperature further in systematic matter, not just as a source of error. In chapter 5 the results of a series of TG measurements that have been performed over a range of temperatures $\left(30.5^{\circ} \mathrm{C}-\right.$ $38.5^{\circ} \mathrm{C}$ ) are presented. Since temperature control and stability of the device appeared not to be up to the required standards, it was decided that a technical amelioration imposed itself. On purpose the technical details are not discussed in chapter 5 but in an appendix, as they are of a very different nature than the rest of the chapter. It is nevertheless vital to illustrate the intricacies of temperature control to fully understand why the current method is not sufficient.

The influence of temperature on TG was measured in normal pooled plasma (NPP) and in defibrinated NPP, in order to find an answer to the hypothesis weather or not TG is diffusion controlled. The assumption was that prothrombin conversion in the presence of fibrin is diffusion limited and therefore much less sensitive to lowering of temperature than thrombin inactivation ${ }^{40}$. In reality the opposite seems to be true with the defibrinated plasma showing much less of response to temperature changes, $+0.001 \% /{ }^{\circ} \mathrm{C}$ for the peak values and $-1.4 \% /{ }^{\circ} \mathrm{C}$ for the endogenous thrombin potential (ETP). NPP plasma on the other hand is more influenced by temperature with a decrease of $-3.2 \% /{ }^{\circ} \mathrm{C}$ for the peak and $4.4 \% /{ }^{\circ} \mathrm{C}$ for the ETP.

The observation that TG is decreasing with increasing temperature is counter intuitive as most (bio)chemical reactions increase with temperature. TG however consists of both pro- and anticoagulant process and as the data in chapter 5 illustrate the procoagulant reactions are less influenced by temperature as compared to the anticoagulant reactions. If both these processes would be influenced in the same way by temperature, TG would be temperature insensitive. However, the combination of a lower overall prothrombin conversion with an even 
more decreased activity of the antithrombins explain a higher apparent thrombin generation. This difference in response between pro- and anticoagulant responses can potentially be explained by the original hypothesis of diffusion-controlled mechanisms of thrombin formation. Unfortunately, experimental constraints in determining accurate decay constants and the lack of sensitivity of the measurement do not allow to either support or falsify this hypothesis.

An important practical point is the influence of temperature on the calibration experiment, that differs markedly from that on thrombin generation. The velocity of substrate conversion by the calibrator increases with increasing temperature and unlike thrombin generation, that decreases. This increases the temperature dependency of the thrombin generation curve that results from the comparison with the calibrator.

Inspection of a calibration curve immediately shows that it is far from being a straight line. Because of this non-linearity the calibration factor that converts the observed reaction velocity into an enzyme concentration changes with the level of fluorescence. Two mechanisms are thought to cause this phenomenon: Firstly, the inner filter effect (IFE) which makes that the fluorescence intensity decreases proportionally to the increasing concentration of fluorescent product. Secondly, substrate consumption (SC), which causes the reaction velocity to decrease proportionally with enzyme concentration as the reaction proceeds. In the CAT-TG an algorithm is used that compares the fluorescence curve from the calibrator well with the TG well and corrects for IFE and SC $\left(\mathrm{see}^{39}\right)$. This algorithm is not based on insight in the mechanisms (IFE and SC) and therefore could be a source of error. In chapter 6 these phenomena that cause the non-linearity of the calibration reaction of TG are assessed and a calculation method is presented based on the insight into the mechanisms.

The relation between the measured fluorescence $\left(\mathrm{F}_{\text {exp }}\right)$ and the concentration of the fluorophore (C) is known to be: $\mathrm{F}=A C e^{-b C}$ with $\mathrm{F}=$ fluorescence, $\mathrm{C}=$ concentration and $\mathrm{A}$ and $\mathrm{b}$ constants. We found that within the range of fluorescence that we encounter in our experiments this formula can be replaced by $F_{\text {exp }}=F_{\max }\left(1-e^{-k C}\right)$. We show that the latter formula, in contrast to the first allows to develop a simple expression for the correction of the inner filter effect: $F_{\text {ideal }}=-F_{\max } \ln \left(1-F_{\text {exp }} / F_{\max }\right)$ (Chapter 6$)$. This formula is generally applicable and is based on the optics of the inner filter effect.

In any fluorometer of the type used for the CAT-TG, this formula can be used after normalization of the fluorescence intensity at $300 \mu \mathrm{M} \mathrm{AMC}$ to $1000 \mathrm{AU}$ and using 2090 as the value of $F_{\max }$ or, alternatively, using 2.09 times the fluorescence intensity at $300 \mu \mathrm{M} \mathrm{AMC}$ for $F_{\max }$. This operation will work as long as all other 
variables of the lambert-beer equation $\left(A=\log _{10}\left(\frac{I_{0}}{I}\right)=\varepsilon . I . c\right)$ stay the same. Mind that the intensity of fluorescence at $300 \mu \mathrm{M}$ is not a constant for a given fluorometer but changes with the intensity of the excitation light source, which diminishes with time. The fluorescence intensity at $300 \mu \mathrm{M}$ AMC should therefore be regularly controlled.

The experiments presented in chapter 6 show that the conversion of substrate by $\alpha_{2} \mathrm{M}-\mathrm{T}$ can be described by the classical Michaelis-Menten hyperbola $\frac{d P}{d t}=$ $E . k_{\text {cat }} . S /(K m+S)$, so that we can calculate the relation between reaction velocity and enzyme concentration as $E=\left(\frac{d P}{d t}\right)(K m+S) /\left(k_{c a t} . S\right)$, after the IFE has been corrected for with the abovementioned formula. This relation between reaction velocity and enzyme concentration is independent of a calibrator curve, the calculation only requires that the kinetic constants $\mathrm{Km}$ and $\mathrm{k}_{\text {cat }}$ are known.

The variation in the optical density of the plasma due to the difference in colour of individual plasmas can be as large as $10 \%$, ignoring the plasma colour could introduce considerable experimental variation. Plasma colour being directly related to the optical density can however easily be corrected for with a simple multiplication factor.

The fluorogenic substrate that is used in TG is only soluble in a buffer containing high quantities of Bovine Serum Albumin this implies that the substrate binds to albumin. Given the fact that albumin is the most abundant plasma protein made us question how changes in albumin level could influence a TG- experiment. We therefore investigated the influence of albumin concentrations on the conversion of ZGGR-AMC by thrombin and by $\alpha 2 M-T$ in chapter 7 . This involves measurement of the initial rates of substrate conversion in buffer with different concentrations of albumin. Classical enzyme kinetics requires measurements of initial rates, i.e. measuring in a time frame where substrate consumption is negligible. This however is precisely the time frame in which the measurement in a fluorometer of the type we used is instable. We therefore measured over the time frame in which the product curve is parabolic. A parabolic progress curve implies a linear relation between reaction velocity and substrate concentration. This is to be expected in the beginning of the reaction where the change of $S$ in the numerator of the Michaelis-Menten equation $\left(E . k_{\text {cat }} . S\right)$ does count whereas in the denominator $(K m+S)$ it is still negligible because $K m \gg S$ The initial rate therefore can be calculated as the linear component in a parabola fitted to the first part of the fluorescence trace. This principle is applicable to the measurement of initial rates of calibrator curves in plasma as well. 
We found that albumin has hardly an influence on the kinetic constants of thrombin but a large influence of the $\mathrm{Km}$ of $\alpha 2 \mathrm{M}-\mathrm{T}$. This we explained by assuming that the substrate binds reversibly to albumin but that thrombin is capable of splitting the bound substrate whereas $\alpha 2 \mathrm{M}-\mathrm{T}$ is not. 


\section{Substrate concentration}

In chapter 8 an assessment of the influence of the fluorogenic ZGGR-AMC substrate on the TG experiment was investigated in a concentration dependent manner. The presence of the substrate causes a fraction of the thrombin to be substrate bound to its active centre. Therefore, less thrombin is available to interact with its inhibitors antithrombin (AT) and $\alpha_{2}$ Macroglobulin $\left(\alpha_{2} M\right)$. Indeed, substrate was found to increase TG values because of this competition. Splitting the TG curve in a prothrombin conversion- and a decay-curve ${ }^{41}$ showed that the main effect of the substrate is on the attenuation of TG by decreasing its half-life time. Naturally, occupation of the active center of thrombin by substrate not only slows down decay but also inhibits positive feedback effects like those on factors $\mathrm{V}, \mathrm{VIII} \mathrm{XI}$ and negative effects after binding of thrombin to thrombomodulin. This, theoretically, calls for using minimal substrate concentrations. The original choice for the concentration of the thrombin substrate was dictated more by solubility than any other consideration (chapter 7).

In chapter 4 we lowered the substrate concentration of the experiments to 150 $\mu \mathrm{M}$ and found no adverse effects on the thrombin generation curves. At this concentration no considerable effect of substrate depletion was observed as the end level fluorescence of the TG experiment was about $65 \%$ of the maximum fluorescence, like in experiments at higher substrate concentrations. It thus appears that substrate consumption is roughly proportional to the inhibition of thrombin decay by the substrate.

Now the question poses itself whether we can suggest amelioration of current practice of TG-measurement in the light of our results, either by adaptations that relate to calibration, to the fluorogenic substrate, to the calculation method and to technical ameliorations.

\section{Calibration}

Calibration is currently a necessity to be able to quantify the amount of thrombin generated and to correct for the specific colour of the plasma as well as for the inner filter effect and substrate consumption. The calibration experiment is run in parallel in a separate experimental well. Even at the highest level of scrutiny an increase of experimental error must be introduced by comparison of two experiments and the calculations that this involves. The most adequate solution for this would be to abolish the need for an "external" calibration experiment and internalize it. It has been suggested by Hemker (A self-calibrated thrombin generation assay. European patent application P6066184EP) that it is possible to use an "internal" ratio for the calibration. When a small fixed amount of product is added to the reaction mixture before the start of the TG reaction, and when the inner filter effect is compensated for so that fluorescence is proportional to 
product concentration, then the progress of the reaction can be expressed as the ratio of the level of fluorescence to the initial level. $r_{\text {exp }}=\left(\left(F_{\text {exp }} / F_{0}\right)-1\right)$. This already compensates for effects of the colour of plasma etc. Also, this allows to express the progress of the reaction in terms of the fraction of substrate consumed. From the present work we know that simple Michaelis-Menten kinetics apply, so it must be possible to calculate, at each level of substrate consumption, the amount of enzyme (thrombin) that causes the measured reaction velocity.

\section{The fluorogenic substrate.}

The substrate has a pronounced effect on the development of the TG curve as the substrate competes for thrombin with the natural substrates that are present in the plasma. We have shown the main effects to be in the thrombin decay phase and to a lesser extend in the thrombin formation phase. Moreover, we have also shown that it is possible to correct for the competitive effect of the substrate. Ideally a substrate with very high $\mathrm{Km}$ should be used of which only a small fraction is consumed. In that case $\mathrm{Km}+\mathrm{S}$ remains practically constant during the experiment and the reaction velocity is simply proportional to enzyme concentration and substrate concentration, which, in combination with the ratio method mentioned above would allow simple direct determination of the TG-curve.

Substrates that are not split by the $\alpha 2 \mathrm{M}-\mathrm{T}$ complex have many advantages: less substrate will be consumed and the inner filter effect would be less important, as the overall fluorescent trace would remain relatively low. The calculation step required to eliminate the contribution of the $\alpha 2 \mathrm{M}-\mathrm{T}$ complex to the total amidolytic activity would become superfluous, so that it would be easier to determine the end of the TG experiment.

In chapter 4 we highlight the main bottleneck of the $\alpha 2 \mathrm{M}-\mathrm{T}$ macroglobulin correction. In the experimental procedure, the course of total amidolytic activity $f_{a}(t)$ is found, consisting of free thrombin $f_{g}(t)$ and $\alpha 2 \mathrm{M}-\mathrm{T}$. Because the velocity of formation of $\alpha 2 \mathrm{M}-\mathrm{T}$ in the pseudo first order reaction $\alpha 2 \mathrm{M}+\mathrm{T} \rightarrow \alpha 2 \mathrm{M}-\mathrm{T}$ is proportional to the concentration of free thrombin, the total amount of $\alpha 2 \mathrm{M}-\mathrm{T}$ formed is $k$ times the integral of the thrombin-time curve. It is therefore seemingly easy to estimate the contribution of $\alpha 2 \mathrm{M}-\mathrm{T}$ to the total amidolytic activity. In practice however there is one glitch, because in order to be able to determine this constant a point in time has to be found where all free thrombin has disappeared. The key element in this process is that if $k$ is underestimated, part of the activity of $\alpha_{2} \mathrm{M}-\mathrm{T}$ is attributed to free thrombin and contributes to the ETP, whereas when $k$ is overestimated part of the free thrombin is attributed to $\alpha_{2} \mathrm{M}-\mathrm{T}$. With use of the current substrate no satisfiable solution to end level determination has been found yet. 
Besides the perfect substrate, which might be challenging to synthesise, gains can already be made by altering the current substrate concentration. Lowering to substrate concentration to e.g. $150 \mu \mathrm{M}$ would not only reduce the inner filter effect but also benefits the TG experiment as there is less competition between the substrate and the serpins inhibiting TG.

\section{Technical ameliorations to the device.}

An obvious improvement would be the level of accuracy of the temperature control within the TG device. Whatever the temperature of the measurement will be, there is still a need for accurate temperature control as measuring at room temperature is not an option. The inter-laboratory temperature differences as well as seasonal variations in the laboratory would then prove to be new sources of error. A second adaptation would be the excitation light source, the stability of the intensity of the light source is of vital importance especially when a standard correction factor for the inner filter effect is to be used. Only LED's fulfil these requirements because they are stable throughout their lifetime. Both tungstenhalogen and mercury/xenon arc lamps, the only reasonable alternatives, show a decay with time and they have considerable pre-heating times during which their output intensity is highly variable.

In short: The CAT-TG test that is the most used at the moment and, in a slightly modified form, a candidate for clinical application is essentially the same as that proposed by Hemker et al. almost twenty years ago. Hundreds of articles have shown its potential use in clinical and fundamental research and promise it to be useful in patient care. In this thesis we show that fundamental ameliorations of the original design are possible and desirable. 


\section{References}

1. Hemker, H. C. et al. Calibrated Automated Thrombin Generation Measurement in Clotting Plasma. Pathophysiol. Haemost. Thromb. 33, 4-15 (2003).

2. Dargaud, Y., Lienhart, A. \& Negrier, C. Prospective assessment of thrombin generation test for dose monitoring of bypassing therapy in hemophilia patients with inhibitors undergoing elective surgery. Blood 116, 5734-5737 (2010).

3. Millet, A. et al. Thrombin generation in patients with acquired haemophilia and clinical bleeding risk. Br. J. Haematol. 153, 136-139 (2011).

4. Bosch, Y. P. J. et al. Measurement of thrombin generation intra-operatively and its association with bleeding tendency after cardiac surgery. Thromb. Res. 133, 488-494 (2014).

5. Bloemen, S., Zwaveling, S., Ten Cate, H., Ten Cate-Hoek, A. \& de Laat, B. Prediction of bleeding risk in patients taking vitamin $\mathrm{K}$ antagonists using thrombin generation testing. Plos One 12, e0176967 (2017).

6. Tans, G. et al. Activated protein C resistance determined with a thrombin generation-based test predicts for venous thrombosis in men and women. Br. J. Haematol. $122,465-470$ (2003).

7. Hron, G., Kollars, M., Binder, B. R., Eichinger, S. \& Kyrle, P. A. Identification of patients at low risk for recurrent venous thromboembolism by measuring thrombin generation. JAMA 296, 397-402 (2006).

8. Buyue, Y., Whinna, H. C. \& Sheehan, J. P. The heparin-binding exosite of factor IXa is a critical regulator of plasma thrombin generation and venous thrombosis. Blood 112, 3234-3241 (2008).

9. Sanchez, C., Alessi, M. C., Saut, N., Aillaud, M. F. \& Morange, P. E. Relation between the antithrombin Cambridge II mutation, the risk of venous thrombosis, and the endogenous thrombin generation. J. Thromb. Haemost. JTH6, 1975-1977 (2008).

10. Lavigne-Lissalde, G. et al. Prothrombin G20210A carriers the genetic mutation and a history of venous thrombosis contributes to thrombin generation independently of factor II plasma levels. J. Thromb. Haemost. JTH 8, 942-949 (2010).

11. Ay, C. et al. Prediction of venous thromboembolism in patients with cancer by measuring thrombin generation: results from the Vienna Cancer and Thrombosis Study. J. Clin. Oncol. Off. J. Am. Soc. Clin. Oncol. 29, 2099-2103 (2011).

12. Anžej Doma, S., Vučnik, M., Božič Mijovski, M., Peternel, P. \& Stegnar, M. Enhanced thrombin generation in women with a history of oral contraception-related venous thrombosis. Thromb. Res. 132, 621-626 (2013).

13. Hylckama Vlieg, A. et al. The risk of a first and a recurrent venous thrombosis associated with an elevated D-dimer level and an elevated thrombin potential: results of the THE-VTE study. J. Thromb. Haemost. 13, 1642-1652 (2015).

14. Martin-Fernandez, L. et al. Genetic Determinants of Thrombin Generation and Their Relation to Venous Thrombosis: Results from the GAIT-2 Project. PloS One 11, e0146922 (2016).

15. Santagostino, E. et al. Severe hemophilia with mild bleeding phenotype: molecular characterization and global coagulation profile. J. Thromb. Haemost. 8, 737-743 (2010). 
16. Maurer, E. et al. Targeting Platelet GPIb $\beta$ Reduces Platelet Adhesion, GPIb Signaling and Thrombin Generation and Prevents Arterial ThrombosisSignificance. Arterioscler. Thromb. Vasc. Biol. 33, 1221-1229 (2013).

17. Ten Cate, H. \& Hemker, H. C. Thrombin Generation and Atherothrombosis: What Does the Evidence Indicate? J. Am. Heart Assoc. 5, (2016).

18. Tosi, F. et al. Increased plasma thrombin potential is associated with stable coronary artery disease: An angiographically-controlled study. Thromb. Res. 155, 16-22 (2017).

19. Nagashima, H. Studies on the different modes of action of the anticoagulant protease inhibitors DX-9065a and Argatroban. I. Effects on thrombin generation. J. Biol. Chem. 277, 50439-50444 (2002).

20. Walker, C. P. R. \& Royston, D. Thrombin generation and its inhibition: a review of the scientific basis and mechanism of action of anticoagulant therapies. Br. J. Anaesth. 88, 848-863 (2002).

21. Brocal, I., Marco, P., Lucas, J., Verdú, J. \& Tarín, F. Thrombin generation test in patients under anticoagulant therapy with vitamin K antagonists. Thromb. Haemost. 101, 594-595 (2009).

22. Robert, S. et al. Is thrombin generation the new rapid, reliable and relevant pharmacological tool for the development of anticoagulant drugs? Pharmacol. Res. 59, 160-166 (2009).

23. Bloemen, S., Hemker, H. C. \& Al Dieri, R. Large inter-individual variation of the pharmacodynamic effect of anticoagulant drugs on thrombin generation. Haematologica 98, 549-554 (2013).

24. Chowdary, P. et al. Thrombin generation assay identifies individual variability in responses to low molecular weight heparin in pregnancy: implications for anticoagulant monitoring. Br. J. Haematol. 168, 719-727 (2015).

25. Tripodi, A. et al. How the direct oral anticoagulant apixaban affects thrombin generation parameters. Thromb. Res. 135, 1186-1190 (2015).

26. D’Alessio, A. et al. Long Term Low Molecular Weight Heparin Anticoagulant Therapy Modulates Thrombin Generation and D-dimer in Patients with Cancer and Venous Thromboembolism. Cancer Invest. 35, 490-499 (2017).

27. Reverter, J. C. et al. Inhibition of platelet-mediated, tissue factor-induced thrombin generation by the mouse/human chimeric 7E3 antibody. Potential implications for the effect of c7E3 Fab treatment on acute thrombosis and 'clinical restenosis'. J. Clin. Invest. 98, 863-874 (1996).

28. Keularts, I. M., Béguin, S., de Zwaan, C. \& Hemker, H. C. Treatment with a GPIIb/IIla antagonist inhibits thrombin generation in platelet rich plasma from patients. Thromb. Haemost. 80, 370-371 (1998).

29. Kost, G. J. et al. Point-of-Care Testing for Disasters: Needs Assessment, Strategic Planning, and Future Design. Clin. Lab. Med. 29, 583-605 (2009).

30. Koch, K. et al. Thrombin generation test in microfluidic systems. J. Appl. Phys. 105, 102012 (2009).

31. Ten Cate, H. Thrombin generation in clinical conditions. Thromb. Res. 129, 367370 (2012).

32. Ninivaggi, M. et al. Whole-Blood Thrombin Generation Monitored with a Calibrated Automated Thrombogram-Based Assay. Clin. Chem. 58, 1252-1259 (2012). 
33. Perrin, J. et al. Large external quality assessment survey on thrombin generation with CAT: further evidence for the usefulness of normalisation with an external reference plasma. Thromb. Res. 136, 125-130 (2015).

34. Dargaud, Y. et al. Effect of standardization and normalization on imprecision of calibrated automated thrombography: an international multicentre study. Br. J. Haematol. 139, 303-309 (2007).

35. Dargaud, Y. et al. Standardisation of thrombin generation test - which reference plasma for TGT?: An international multicentre study. Thromb. Res. 125, 353-356 (2010).

36. Loeffen, R. et al. Preanalytic variables of thrombin generation: towards a standard procedure and validation of the method. J. Thromb. Haemost. JTH 10, 2544-2554 (2012).

37. Dargaud, Y. et al. Evaluation of a standardized protocol for thrombin generation measurement using the calibrated automated thrombogram: An international multicentre study. Thromb. Res. 130, 929-934 (2012).

38. Hemker, H. C., Hemker, P. W., Al Dieri, R. \& others. The technique of measuring thrombin generation with fluorescent substrates: 4. The H-transform, a mathematical procedure to obtain thrombin concentrations without external calibration. Thromb. Haemost. 101, 171-177 (2009).

39. Hemker, H. C. \& Kremers, R. Data management in Thrombin Generation. Thromb. Res. 131, 3-11 (2013).

40. Hemker, H. C., De Smedt, E. \& Hemker, P. W. During coagulation, thrombin generation shifts from chemical to diffusional control. J. Thromb. Haemost. 3, 2399-2400 (2005).

41. Kremers, R. M. W., Peters, T. C., Wagenvoord, R. J. \& Hemker, H. C. The balance of pro- and anticoagulant processes underlying thrombin generation. J. Thromb. Haemost. 13, 437-447 (2015). 

